\title{
Consumer Evaluation of Low Sodium Mozzarella Cheese and Development of a Novel Method for Evaluating Emotions
}

\author{
A Thesis Presented to the Faculty of \\ California Polytechnic State University
}

San Luis Obispo

In Partial Fulfillment of the Requirements for the Degree of

Master of Science in Agriculture, with Specialization in Food Science and Nutrition

By Lauren Alyse Collinsworth

January 2013 
(C) 2013

Lauren Alyse Collinsworth

ALL RIGHTS RESERVED 


\title{
COMMITTEE MEMBERSHIP
}

TITLE: $\quad$ Consumer Evaluation of Low Sodium Mozzarella Cheese and

\section{Development of a Novel Method for Evaluating Emotions}

\author{
AUTHOR: $\quad$ Lauren Alyse Collinsworth
}

DATE SUBMITTED: January 2013

COMMITTEE CHAIR: Amy Lammert, PhD

Assistant Professor, Food Science and Nutrition

COMMITTEE MEMBER: Nana Farkye, PhD

Professor, Dairy Science

COMMITTEE MEMBER: Amanda Lathrop, $\mathrm{PhD}$

Assistant Professor, Food Science and Nutrition

COMMITTEE MEMBER: Karen McGaughey, PhD

Associate Professor, Statistics 


\title{
ABSTRACT \\ Consumer Evaluation of Low Sodium Mozzarella Cheese and Development of a Novel Method for Evaluating Emotions
}

\author{
Lauren Alyse Collinsworth
}

Mozzarella cheese is currently the highest consumed cheese in the United States. The popularity of mozzarella cheese is typically attributed to the high consumption rates of pizza cheese and string cheese; both of which are low moisture part skim (LMPS) mozzarella cheese. A single serving of LMPS mozzarella cheese contains approximately $8 \%$ of the daily value (DV) for sodium, a mineral which is currently consumed in excess among most Americans. On average, one in three Americans has hypertension. This condition is strongly associated with excessive sodium intake, and it is a leading risk factor for cardiovascular disease and stroke in the United States. Considering the popularity of LMPS mozzarella cheese, its sodium content, and the alarmingly high rates of hypertension among the American population, mozzarella cheese appears to be a product worth pursuing for sodium reduction. Salt $(\mathrm{NaCl})$ provides several key benefits to cheese including: flavor enhancement, preservation, moisture control, and syneresis; thus reducing its concentration in cheese can result in multiple quality concerns.

Previous research has investigated the effects of lower sodium in a variety of cheeses including: cheddar, feta, and imitation cheese. Typical methods for reducing sodium content in cheese include reduction of $\mathrm{NaCl}$ alone to a level which is still acceptable or partial substitution of $\mathrm{NaCl}$ with salt replacers including $\mathrm{KCl}$. For assessing the consumer acceptability of low sodium cheeses, researchers have typically employed 
the use of traditional hedonic, preference, and ranking questions; however, consumer scientists have recently suggested the benefits of asking consumers questions which go beyond typical acceptability questions. Purchase intent and decision making have been associated with consumer emotions, and perhaps by better understanding consumer emotions toward nutritional alternative foods, including low sodium mozzarella cheese, a more successful low sodium cheese can be developed.

The current study implemented a series of tests to progressively understand the role $\mathrm{NaCl}$ plays in mozzarella cheese and consumer opinion of low sodium cheese. A series of traditional sensory tests, including triangle, duo-trio, and hedonic tests, were performed to determine a sensory transparent antimicrobial and a consumer acceptable salt replacer. Based upon this sequence of tests, a single antimicrobial (SEA-i F75) and concentration $(0.275 \%)$ were selected in addition to the most consistently preferred salt replacer in a low sodium mozzarella cheese system; however, the most preferred salt replacer was dependent upon the type of mozzarella cheese (direct acid or bacteria cultured).

In order to address the potentially limited information from traditional sensory testing, a novel method for evaluating emotions was developed. The IMET (Image Measurement of Emotion and Texture) method utilized consumer provided images of emotions, researcher generated emotion images, and emotion words (the current industry standard) to aid in emotion testing, and the use of texture images and texture words (industry standard) for texture assessment. The IMET method was tested and validated across three commercial food product categories: orange soda, dairy beverages, and convenience cheeses. The IMET study indicated consumer selected emotion images were 
less variable than emotion words in a positive emotion, but the words only method was less variable in a negative emotion. Additionally, subjects were more likely to use images of themselves for positive emotions, and images of others for the negative emotions.

After validation of the IMET method, the consumer emotion images method was used in conjunction with consumer acceptability testing and instrumental texture analysis in non-commercial low sodium mozzarella cheese. This study indicated the full sodium cheese was consistently liked most, followed by the $100 \% \mathrm{KCl}$ cheese sample. Additionally, cheese with higher hedonic scores had increased changes in the positive emotions, while the disliked products had increased changes in the negative emotions across the eating experience. The instrumental texture analysis resulted in significant textural differences between the eight samples tested, and samples with higher mean scores for all texture attributes were associated with having higher mean hedonic scores.

The studies performed in this thesis are important contributions for better understanding the role of $\mathrm{NaCl}$ in LMPS mozzarella cheese, and the consumer's perception and potential acceptance of this nutritional alternative product. Additionally, the development of a novel emotion testing method may impact how researchers ask consumer questions, conduct consumer research, and investigate the effects of images on emotion testing with consumers.

Keywords: low sodium mozzarella cheese, consumer testing, emotional responses, texture analysis, images 


\section{ACKNOWLEDGEMENTS}

I would like to thank my entire committee, Dr. Amy Lammert, Dr. Nana Farkye, Dr. Amanda Lathrop, and Dr. Karen McGaughey, for their endless guidance, support, passion and knowledge; I'm truly thankful for the time and effort you have put into helping me grow and learn throughout graduate school. To Dr. Lammert, a special thank you, for all the amazing opportunities you have provided me, I am forever grateful.

Thank you to all the wonderful graduate students at the Dairy Products Technology Center for your knowledge, entertainment, and support; I am so thankful to have met you all. Thank you to Matt Arnold and Katy Lees for your help and guidance.

I am very appreciative of the financial support provided by Dairy Management Inc. (DMI) and the California State University Agricultural Research Initiative (CSU ARI).

I would like to finally acknowledge the unfailing love, support, and inspiration of my family, thank you for everything you all have done. 


\section{TABLE OF CONTENTS}

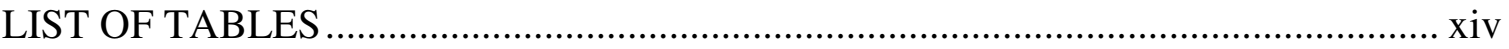

LIST OF FIGURES …………………............................................................. xvii

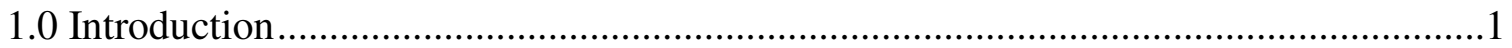

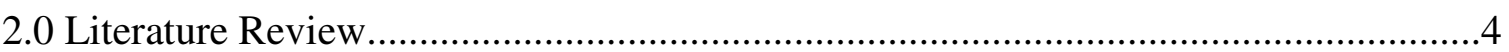

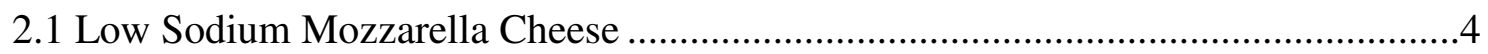

2.1.1 Reducing Sodium Intake.............................................................................

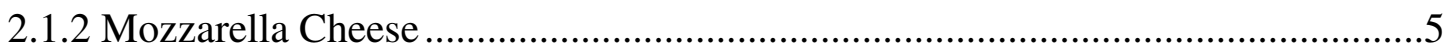

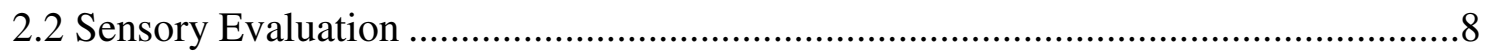

2.2.1 Traditional Sensory Testing ...........................................................................

2.2.1.1. Affective Testing ...................................................................................

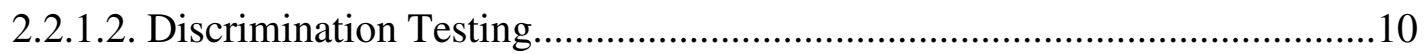

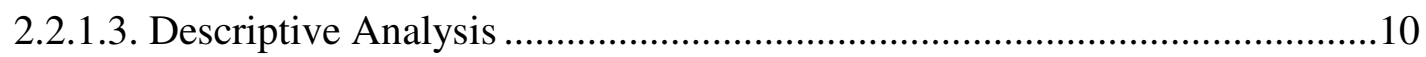

2.2.2 Current Sensory Evaluation of Low Sodium Cheeses .......................................12

2.2.2.1. Decrease $\mathrm{NaCl}$ Concentration...................................................................13

2.2.2.2. Substitution of $\mathrm{NaCl}$..............................................................................

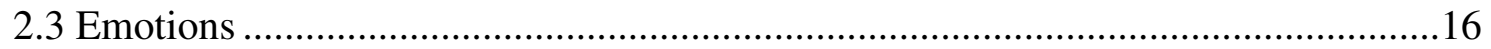

2.3.1 Why Evaluate Emotions?..................................................................................

2.3.2 Decision Making and Reward Mechanisms ......................................................19

2.3.3 Brain Relationship between Emotion, Taste, Texture, and Images .....................22

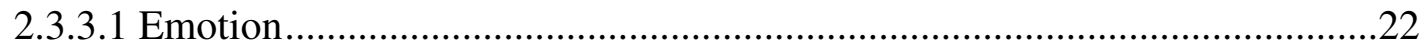

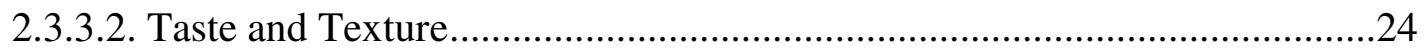

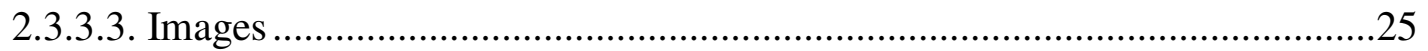

2.3.4 Current Emotion Testing Methods........................................................................28

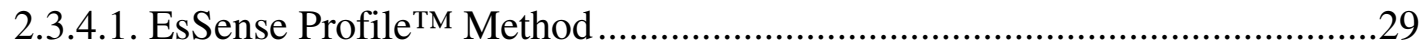




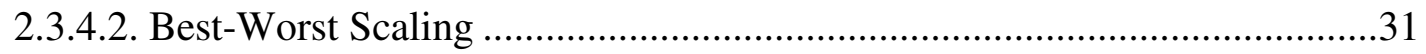

2.3.4.3. Product Emotion Measure (PrEmo) ...................................................................34

2.3.4.4. Eliciting Emotions with Odors and Pictures ...................................................36

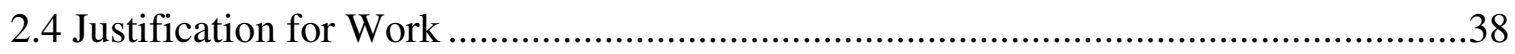

3.0 Sensory Evaluation of Antimicrobials and Salt Replacers in Low Sodium

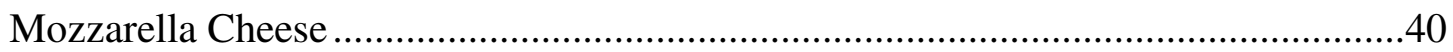

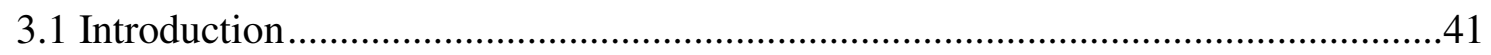

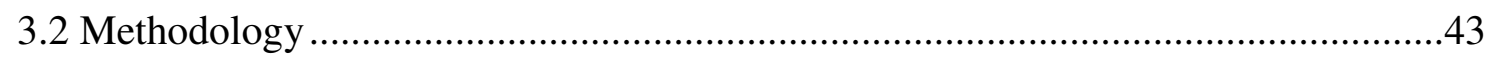

3.2.1 Materials: Antimicrobials ................................................................................

3.2.2 Methods: Antimicrobials ………………………………………………….....43

3.2.2.1 Triangle Test with Full Sodium Mozzarella Cheese........................................45

3.2.2.2 Triangle Test with Low Sodium Mozzarella Cheese......................................46

3.2.2.3 Duo-Trio Tests with Low Sodium Mozzarella Cheese..................................46

3.2.2.4 Hedonic Testing with Low Sodium Mozzarella Cheese..................................47

3.2.2.5 Effect of Time and Concentration of Antimicrobial Dip on

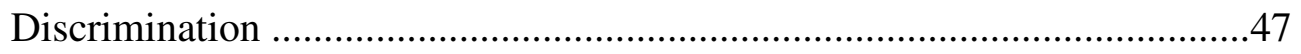

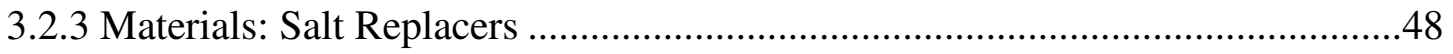

3.2.4 Methods: Salt Replacers ……………………………………………………....49

3.2.4.1 Hedonic and Ranking in Direct Acidified and Bacteria Cultured

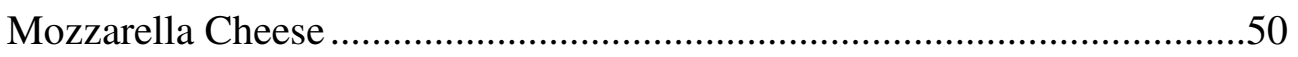

3.2.5 General Sensory Testing Procedures .............................................................50

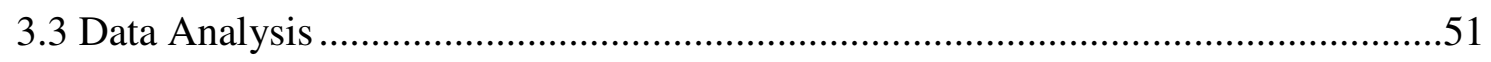

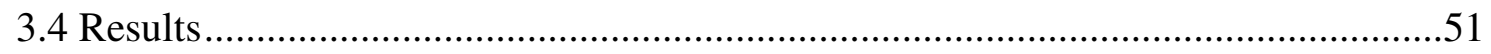

3.4.1 Antimicrobial Sensory Results ............................................................................51

3.4.1.1 Triangle Test with Full Sodium Mozzarella Cheese.......................................51 
3.4.1.2 Triangle Test with Low Sodium Mozzarella Cheese..................................52

3.4.1.3 Duo-Trio with Low Sodium Mozzarella Cheese .......................................54

3.4.1.4 Hedonic Testing with Low Sodium Mozzarella Cheese..............................55

3.4.1.5 Effect of Time and Concentration of Antimicrobial Dip on

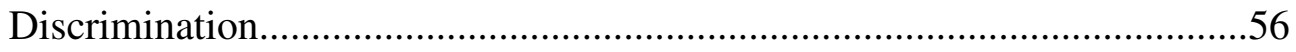

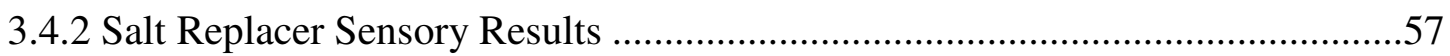

3.4.2.1 Hedonic and Ranking Scores for Direct Acidified Mozzarella

Cheese

3.4.2.2 Hedonic and Ranking Scores for Bacteria Cultured Mozzarella

Cheese

3.5 Discussion .62

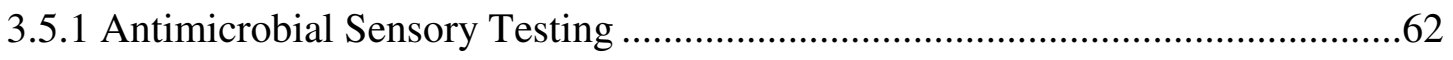

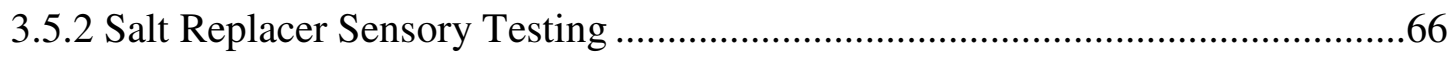

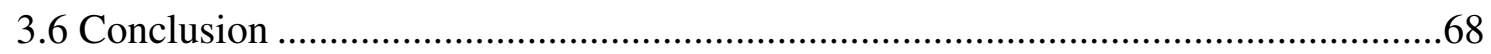

4.0 Development of a Novel Method for Evaluating Emotions and Texture:

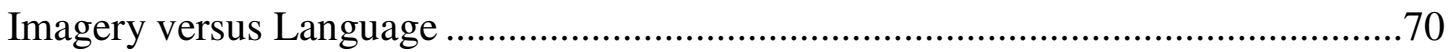

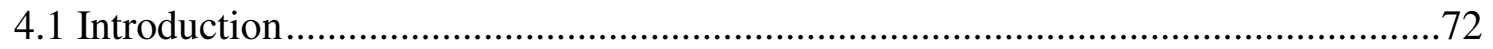

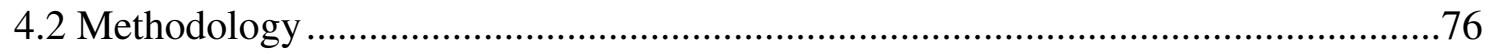

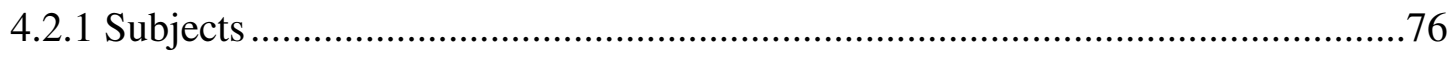

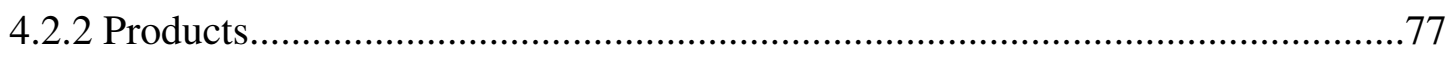

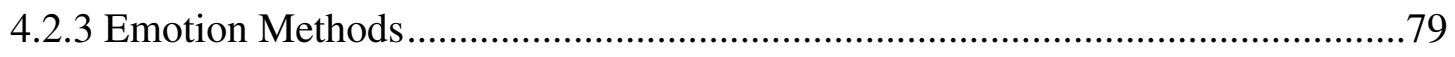

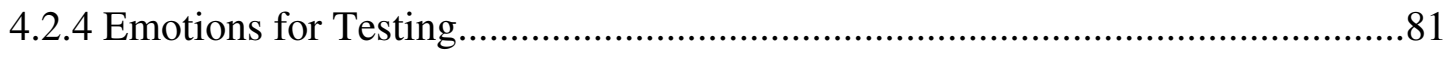

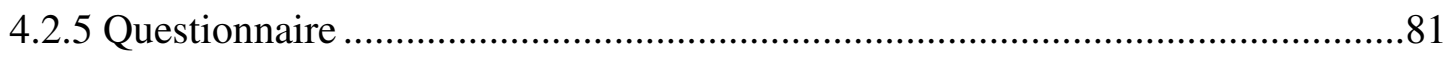

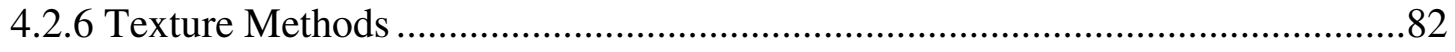

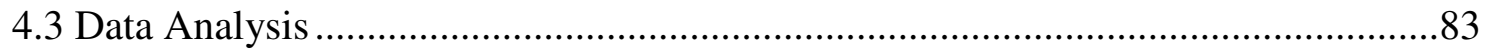




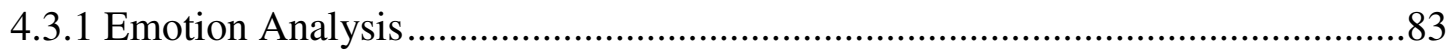

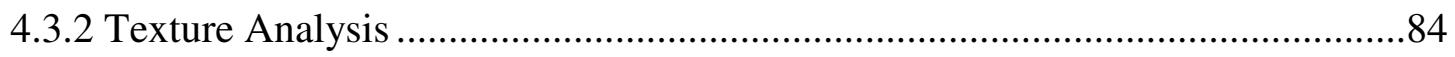

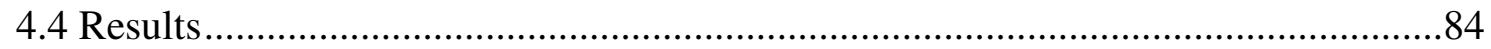

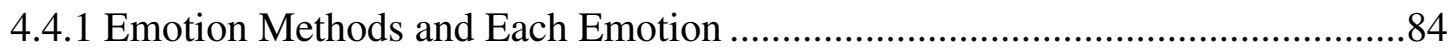

4.4.2 Change in Texture Attributes and Emotions by Texture Methods .......................88

4.4.3 Texture Methods and Change in Emotion .......................................................8

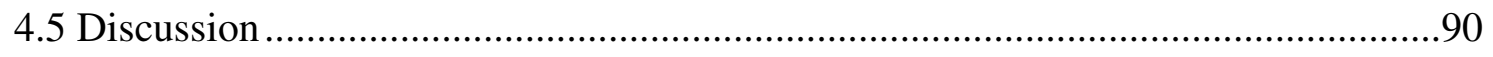

4.5.1 Image Measurement of Emotion and Texture ……..............................................90

4.5.2 Emotion Method Findings ……………………….........................................

4.5.3 Current Emotion Methodologies........................................................................93

4.5.4 Texture Method Findings ..................................................................................96

4.5.5 Current Texture Measurement Methodologies .....................................................98

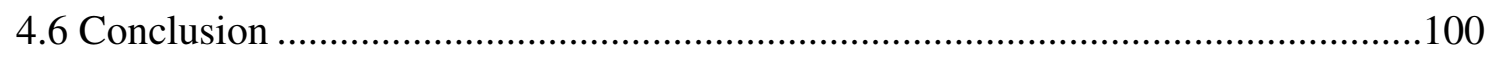

5.0 Evaluating Consumer Emotional Responses and Liking to Non-Commercial

Low Sodium Mozzarella Cheese …………………………………………….......101

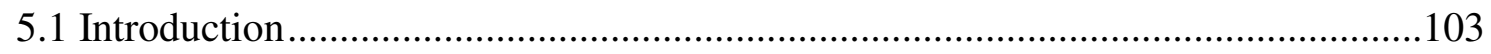

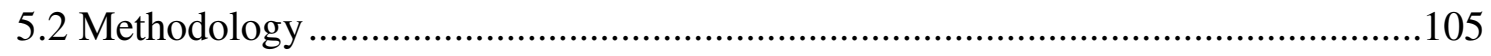

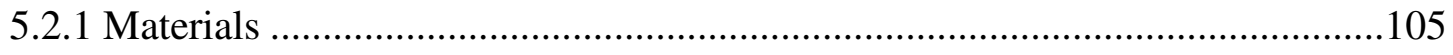

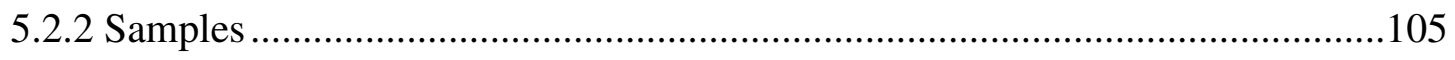

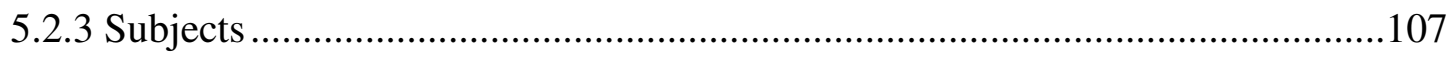

5.2.4 Questionnaire and Testing Procedure ……………….....................................107

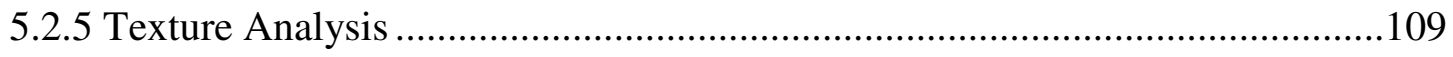

5.3 Data Analysis .................................................................................................110

5.3.1 Product Hedonic Analysis...................................................................................110

5.3.2 Comparison of First, Middle, and End Liking Scores .......................................111 
5.3.3 Liking Score Change between Day 1 and Day 2

5.3.4 Comparison of Each Change in Emotion by Cheese Variable .112

5.3.5 Comparison of First Emotion Scores to End Emotion Scores 113

5.3.6 Emotional Changes between Day 1 and Day 2

5.3.7 Comparison of Change in Emotion by Gender

5.3.8 Texture Analysis 114

5.4 Results. .114

5.4.1 Product Hedonic Analysis 114

5.4.2 Comparison of First, Middle and End Liking Scores 117

5.4.3 Liking Score Change between Day 1 and Day 2 ...... 118

5.4.4 Comparison of Each Change in Emotion by Cheese Variable 119

5.4.5 Comparison of First Emotion Scores to End Emotion Scores 124

5.4.6 Emotional Changes between Day 1 and Day 2 126

5.4.7 Comparison of Change in Emotion by Gender

5.4.8 Texture Analysis 128

5.5 Discussion 130

5.5.1 Product Hedonics 131

5.5.2 Comparison of First, Middle, and End Liking Scores 132

5.5.3 Changes in Liking Scores between Day 1 and Day 2

5.5.4 Comparison of Each Change in Emotion by Cheese Variable 134

5.5.5 Comparison of First Emotion Score to End Emotion Score 135

5.5.6 Emotional Changes between Day 1 and Day 2 136

5.5.7 Comparison of Change in Emotion by Gender 136

5.5.8 Texture Profile Analysis 137

5.5.9 Main Study Objectives and Outcomes 138 
5.6 Conclusion ....................................................................................... 140

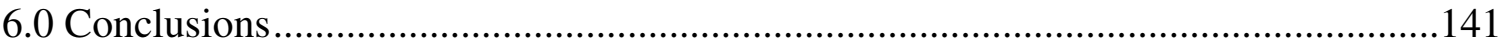

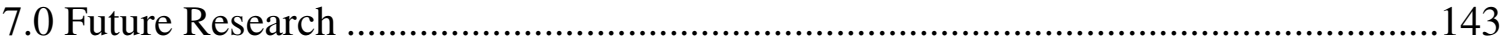

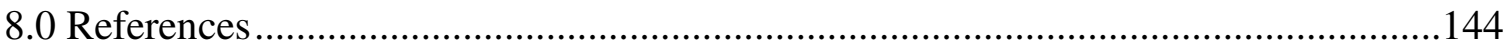




\section{LIST OF TABLES}

Table

Page

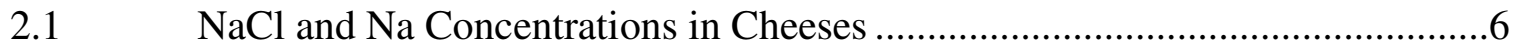

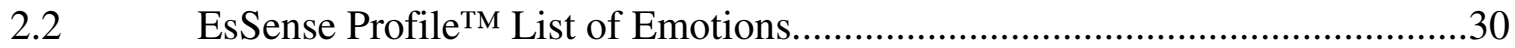

2.3 Best-Worst Scaling List of Emotions ..................................................33

Comparison of Emotion Methods ..................................................................

3.1 Antimicrobial Time and Concentration Parameters ...................................48

S.2 Specifications for Salt Replacer Cheese Variables ....................................49

3.3 Day 1 and Day 2 Sensory Testing with Antimicrobials ..............................52

Session 1: Triangle Test with All Antimicrobials......................................53

3.5 Session 2: Triangle Test with All Antimicrobials.........................................53

Session 1: Duo-Trio Test with All Antimicrobials ..........................................54

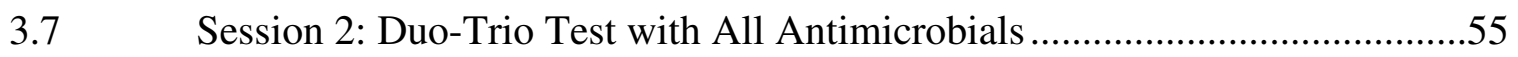

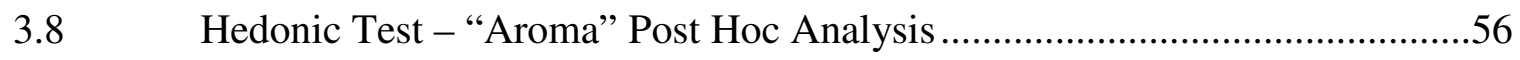

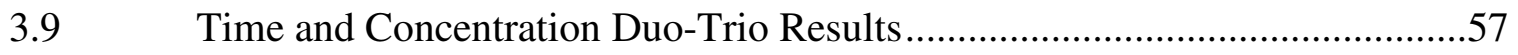

3.10 Direct Acid Hedonic Test Post Hoc Analysis .............................................59

3.11 Direct Acid Ranking Scores Post Hoc Analysis .......................................59

3.12 Bacteria Cultured Hedonic Test Post Hoc Analysis ....................................61

3.13 Bacteria Cultured Ranking Scores Post Hoc Analysis .................................61

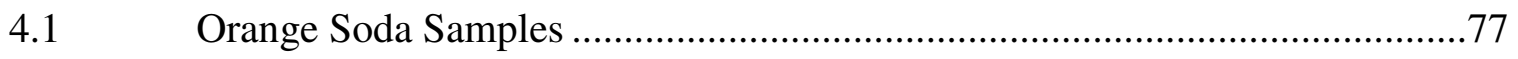

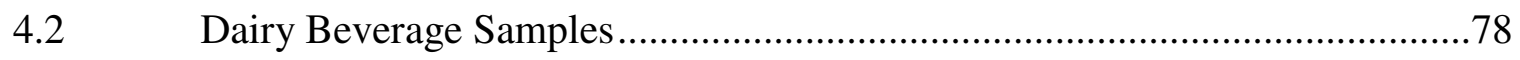

Convenience Cheese Samples................................................................ 78

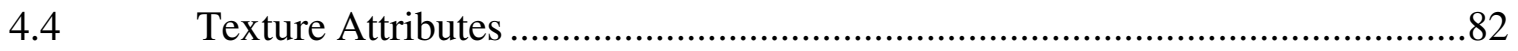

4.5 Change in Caring by Emotion Method ........................................................85

4.6 Change in Sociable by Emotion Method .......................................................86 
4.7 Change in Self Confident by Emotion Method.................................................86

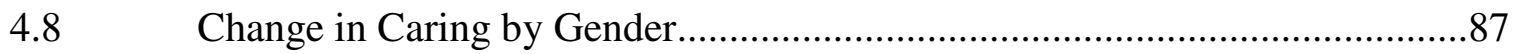

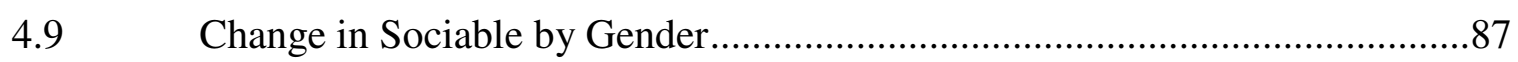

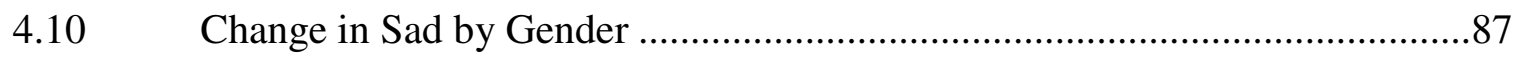

4.11 Number of Post Hoc Significant Differences …………..................................89

5.1 Specifications for Salt Replacer Cheese Variables and Controls ...................106

$5.2 \quad$ Texture Analysis Settings ………………………………………….....110

5.3 Hedonic Attribute Post Hoc Comparison of Day 1 .......................................116

$5.4 \quad$ Hedonic Attribute Post Hoc Comparison of Day $2 \ldots \ldots \ldots \ldots \ldots \ldots \ldots \ldots \ldots \ldots \ldots \ldots \ldots \ldots . . . . .116$

5.5 Change in Overall Flavor Liking Day 1 ……...........................................117

5.6 Change in Saltiness Liking Day 1............................................................117

5.7 Change in Texture Liking Day 1 ............................................................117

5.8 Change in Aroma Liking Day 1 …………….........................................117

5.9 Average Liking Day 1 versus Average Liking Day 2..................................119

5.10 Change in Caring by Variable Day 1 ......................................................119

5.11 Change in Excited by Variable Day 1..........................................................120

5.12 Change in Self Confident by Variable Day 1 ..............................................120

5.13 Change in Fatigued by Variable Day 1 ……..................................................120

5.14 Change in Judgmental by Variable Day 1 ..................................................121

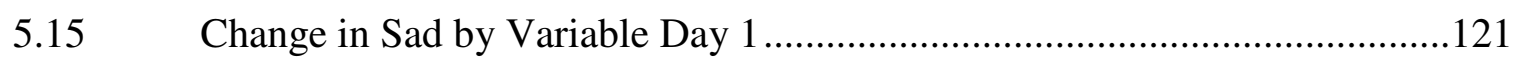

5.16 Change in Caring by Variable Day 2 ………………................................122

5.17 Change in Excited by Variable Day 2 ………….........................................122

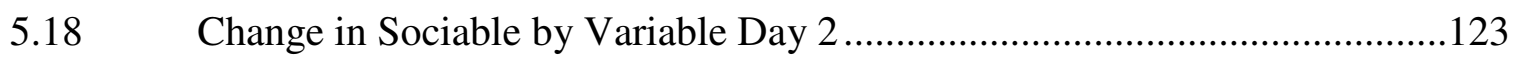

5.19 Change in Self Confident by Variable Day 2 ...............................................123

5.20 Change in Judgmental by Variable Day 2 …..............................................124 


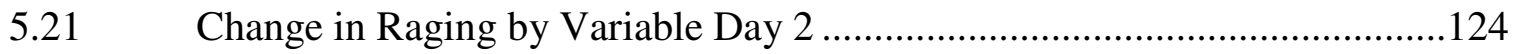

Change in Emotion: First versus End Day 1..........................................125

Change in Emotion: First versus End Day 2 2...........................................126

5.24 Comparing Day 1 Emotion Changes to Day 2 Emotion Changes ..................127

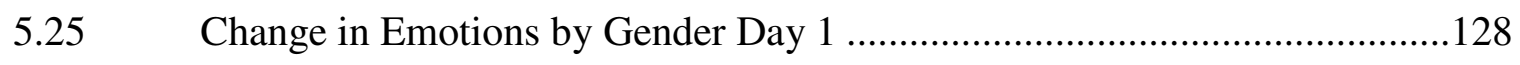

5.26 Change in Emotions by Gender Day 2 ….............................................128

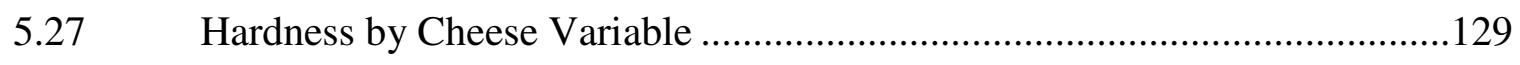

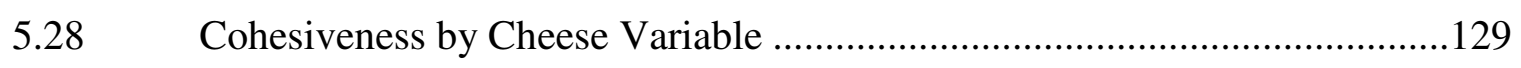

5.29 Gumminess by Cheese Variable .......................................................... 129

Chewiness by Cheese Variable ................................................................ 130 


\section{LIST OF FIGURES}

Figure Page

Example of 9-Point Hedonic Scale ..........................................................

Consumer Perception and Conceptualization of Products............................17

2.3 Decision Making and Reward Mechanism Areas of the Brain.......................20

Sensation Convergence Pathways in the Brain.............................................28

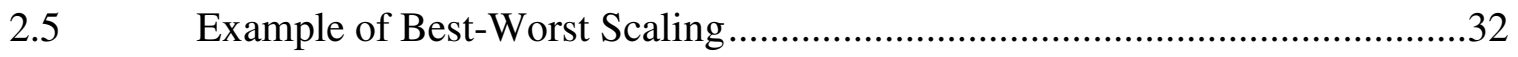

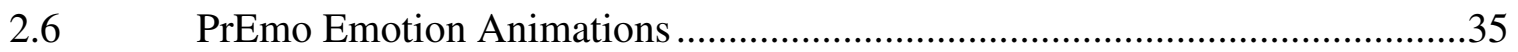

3.1 Overview of Antimicrobial Sensory Testing ........................................ 44

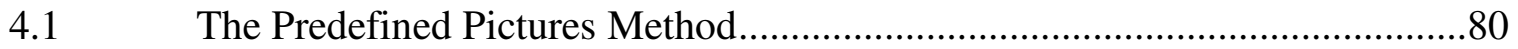

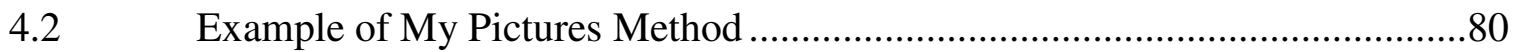

Example of Texture Image Cards ........................................................ 82

4.4 Change in Emotion by Emotion Type and Method .......................................85

4.5 Change in Emotion by Emotion and Method ..........................................90 


\section{Introduction}

Salt $(\mathrm{NaCl})$ has been used as a flavor enhancer and preservative in foods for centuries and is currently found in packaged, processed, and restaurants foods, making it one of the most ubiquitous ingredients in today's food supply (CDC, 2012). Although consumption of sodium ( $\mathrm{Na}$ ) is essential for humans, most Americans consume $\mathrm{NaCl}$ in excess due to its widespread use in a variety of foods. High sodium consumption is associated with hypertension (high blood pressure) which is a leading risk factor for cardiovascular disease and stroke, the primary causes of death in the United States (CDC, 2012). Considering approximately 68 million Americans have hypertension, several initiatives have been created to lower sodium by $25 \%$ in a variety of foods including snacks and convenience products (CDC, 2012; Saltos \& Bowman, 1997).

Mozzarella cheese is the most highly consumed cheese in the United States, with a per capita consumption rate of 11.29 pounds in 2010 (IDFA, 2012). The popularity of mozzarella cheese is often attributed to its use in pizza cheese and string cheese, both of which are low moisture part skim (LMPS) mozzarella cheese. Most cheeses contain a substantial amount of salt, and mozzarella cheese is no exception, containing approximately $1.4-1.8 \%$ sodium; essentially, one stick of string cheese $(\sim 28 \mathrm{~g})$ contributes approximately $8 \%$ of the daily value (DV) of sodium. Considering the consumption rate of mozzarella cheese and its sodium content, LMPS mozzarella appears to be a food product worth pursuing low sodium research and development.

Success of a low sodium mozzarella cheese is dependent on the microbiological and sensory quality of the product. Previous sensory studies have investigated the effects 
of lowering $\mathrm{NaCl}$ only in cheese as well as partial substitution of $\mathrm{NaCl}$ with other salt replacers in a variety of cheeses. Findings have suggested lowering $\mathrm{NaCl}$ levels alone by $25 \%$ are likely to be unsuccessful considering decreasing sodium levels in cottage cheese and cheese sauces by $8 \%$ was detected by consumers (Drake et al., 2011). Partial substitution of $\mathrm{NaCl}$ with other salt replacers, including $\mathrm{KCl}$ have been relatively successful in cheeses including cheddar, feta, and imitation cheeses; however, ratios of $\mathrm{NaCl}$ to $\mathrm{KCl}$ greater than 1:1 have been found to elicit a bitter aftertaste (Cruz et al., 2011; Grummer et al., 2012), therefore the ratio must be carefully monitored in a given cheese system. Previous sensory studies have employed the use of traditional sensory testing methods (hedonic, preference, ranking, etc.); however, consumer and sensory scientists have suggested the benefits of asking consumers questions which go beyond liking with the use of emotion testing (Macht, 1999; King et al., 2010; Desmet et al., 2000; Thomson \& Crocker, 2011).

Testing emotional responses to food products began in the psychology field with abnormal eaters; however, market researchers and consumer scientists have understood the benefits of capturing the consumer's emotional responses to a product. Given this increase in interest with emotion testing, a variety of different emotion testing methods have been developed to easily and accurately capture consumer emotions. Although many emotion methods have been developed, the effectiveness of several of these methods is still being questioned. Most current emotion tests only provide the consumer with a list of emotion words and the consumer is asked to consume or experience a product and indicated which (if any) emotions were elicited (King et al., 2010; Thomson \& Crocker, 2011); however, additional emotion research has suggested a link between 
emotions and images (Holmes et al., 2008; Rolls \& Grabenhorst, 2008; Zaltman \& Coulter, 1995). Perhaps the use of images concurrently with emotion testing would better elicit the emotions used during testing.

The objectives of this thesis were to: 1) conduct a series of traditional sensory tests to determine a sensory transparent antimicrobial and consistently most acceptable salt replacer in low sodium mozzarella cheese, 2) develop and validate a novel method for evaluating emotions with consumers during the eating experience of different food products, and 3) conduct consumer testing with both the traditional consumer testing and the novel emotion testing method to determine if similar emotions could be evoked while consuming low sodium cheese compared to those emotions which are experienced while consuming full sodium mozzarella cheese. Through conducting this progression of traditional consumer and emotion testing, a better understanding of consumer perception and acceptability of low sodium mozzarella cheese may be obtained, and this information can be used to develop a more successful low sodium mozzarella cheese product. 


\section{$\underline{\mathbf{2 . 0}}$}

\section{Literature Review}

\subsection{Low Sodium Mozzarella Cheese}

\subsubsection{Reducing Sodium Intake}

Salt's $(\mathrm{NaCl})$ multifunctional role (i.e. flavor enhancer, preservative, moisture control, etc.) in a variety of food matrices has yielded its ubiquitous presence in a variety of packaged, processed, and restaurant foods within the United States (CDC, 2012) with approximately $75 \%$ of sodium consumed coming from processed foods (USDA, 2005). In terms of human consumption, salt is defined as, "crystallized sodium chloride extracted from natural sources, with the obligatory addition of iodine" (Cruz et al., 2011). Although salt intake is essential for humans for regulating blood pressure, intracellular water transport, and nerve impulse transmission (Cruz et al., 2011), Americans are consuming far beyond the required amount. Due to the abundance of salt in the current food market many Americans are consuming excessive amounts of sodium with an average estimated intake of $3,300 \mathrm{mg}$ of sodium daily, 1,000mg greater than recommended by the U.S. dietary guidelines (CDC, 2012). According to the U.S. Guidelines for Americans (2010), on average individuals with higher sodium intake will have higher blood pressure and individuals with high blood pressure, systolic greater than $140 \mathrm{mmHg}$ or diastolic greater than $90 \mathrm{mmHg}$, (NHLBI, 2012) are at an increased risk of heart disease and stroke, the two leading causes of death in the United States (CDC, 2012). Approximately 68 million (one in three) American adults are estimated to have hypertension (CDC, 2012); given these alarming rates, a variety of initiatives have been 
developed to increase awareness of hypertension and to decrease sodium intake.

Proposals include government funded programs and initiatives within companies such as Pepsi, Dole, Nestle, and General Mills that have been developed to reduce sodium intake by $25 \%$ by 2015 (Saltos \& Bowman, 1997; Fern, 2009).

Although many foods are targeted for decreasing sodium content, this review will focus on the sodium content and reduction with mozzarella cheese. Many cheeses, including mozzarella, are nutrient rich food products, which provide an estimated " $9 \%$ of the protein, $11 \%$ of the phosphorus, and $27 \%$ of the calcium in the U.S. food supply" (Johnson et al., 2009). Despite the significant contribution of calcium via cheese to the food supply, the large proportion of sodium also currently associated with cheese may hinder the bioavailability of calcium; considering a high sodium intake may interfere with calcium bioactivity and increase renal excretion of this mineral (Cruz et al., 2011). The significance for reducing sodium in cheese is threefold: 1) to decrease the consumption of sodium by consumers to help prevent hypertension, cardiovascular disease, and stroke, 2) to help increase retention of cheeses', arguably most significant mineral, calcium, and 3) to aid in increased cheese consumption.

\subsubsection{Mozzarella Cheese}

Cheese consumption rates continue to increase annually, with approximately nine billion pounds of cheese produced annually in the United States, and approximately 3.4 billion pounds being mozzarella cheese alone (USDA, 2010). In 2002, mozzarella cheese surpassed cheddar cheese as the most highly consumed cheese in the U.S. with a suggested per capita consumption rate of 11.29 pounds in 2010 (IDFA, 2012). The recent 
popularity of mozzarella cheese is often attributed to the high consumption of pizza cheese and convenient dairy products such as string cheese, both of which are made with low moisture part skim (LMPS) mozzarella cheese. Mozzarella string cheese has traditionally been marketed as a convenient and healthy snack high in calcium and other milk nutrients (National Dairy Council, 2011), containing approximately $8 \%$ daily value (D.V.) sodium content per serving. Table 2.1 relates mozzarella's sodium content to other common cheeses. Due to the significant consumption rate of LMPS mozzarella cheese in the U.S. and its sodium content, it is an appropriate vehicle for pursuing sodium reduction.

Table 2.1. $\mathrm{NaCl}$ and $\mathrm{Na}$ Concentrations in Cheeses. Approximate $\mathrm{NaCl}$ and $\mathrm{Na}$ concentrations in a variety of cheeses, including mozzarella (Cruz et al., 2011).

\begin{tabular}{|llll|}
\hline Cheese & $\%$ NaCl & $\%$ Na & $\begin{array}{l}\text { Quantity (g) of cheese } \\
\text { equivalent to 100\% } \\
\text { of the recommended } \\
\text { daily intake of sodium }\end{array}$ \\
\hline Quark & 0.15 & 0.06 & 4000 \\
Emmenthal & 0.70 & 0.28 & 857 \\
Brie & 1.60 & 0.63 & 381 \\
Cottage & 1.04 & 0.41 & 585 \\
Gruyere & 0,86 & 0.34 & 706 \\
Provolone & 2.24 & 0.88 & 273 \\
Mozzarella & 1.40 & 0.55 & 436 \\
Cheddar & 1.70 & 0.67 & 358 \\
Gouda & 2.40 & 0.94 & 255 \\
Blue & 3.30 & 1.30 & 185 \\
Parmesão & 4.07 & 1.60 & 150 \\
Roquefort & 4.10 & 1.61 & 149 \\
Romano & 4.10 & 1.61 & 149 \\
Feta & 4.50 & 1.77 & 136 \\
\hline \% = weight $\mathrm{Na}$ or $\mathrm{NaCl} /$ weight $\mathrm{NaCl}$. \\
a Based on the value of recommendation of $\mathrm{NaCl}(2.4$ g) or only \\
Na (6 g). \\
\hline
\end{tabular}

The Code of Federal Regulations (CFR) has specific guidelines for LMPS mozzarella cheese and for making label claims about "low" and "reduced" sodium 
products. 21 CFR 133.156 (a)(1) states in order to be LMPS mozzarella cheese the product must contain at least $45 \%$ milkfat by weight of solids and the moisture content must be greater than $45 \%$ but less than 52\%. Additionally, 21 CFR 133.155 (b)(3)(iii) states that salt is an optional ingredient in mozzarella cheese, suggesting that even when completely removing salt from mozzarella cheese, it is still considered "mozzarella cheese" (Mozzarella cheese and Scamorza cheese, 1993). In regards to "low" and "reduced" sodium claims, 21 CFR 101.61 (4)(i) states to make a "low sodium" claim the food product must have a serving size (less than $30 \mathrm{~g}$ ) with $140 \mathrm{mg}$ or less sodium per serving size and a "reduced sodium" claim as stated by 21 CFR 101.61 (6)(i) requires at least $25 \%$ less sodium per serving size than "an appropriate reference food" (Food Labeling, 2012).

Despite the obvious need for reducing sodium in processed and packed foods, the difficulty in successfully reducing sodium in natural cheeses is its multifunctional beneficial effects including: flavor enhancement, decreasing water activity, enzymatic and culture activity, and syneresis (Grummer et al., 2012; Guinee, 2004). Additionally, consumers often perceive low sodium products as flavorless, boring, and bland (Drake et al., 2010). A variety of studies have been conducted to better understand the consequences on consumer perception and descriptive sensory analysis in lower sodium cheese-type products (Drake et al., 2010; Grummer et al., 2012; Cruz et al., 2011; Katsiari et al., 1997; Fitzgerald \& Buckley, 1985); however, despite the popularity of mozzarella cheese in the U.S., limited information is available regarding low/reduced sodium mozzarella cheese sensory and consumer analysis as a majority of low/reduced sodium cheese work has been focused on cheddar cheese or cheddar-based products 
(Grummer et al., 2012; Drake et al., 2011; Schroeder et al., 1988; Fitzgerald \& Buckley, 1985). Considering the current domestic consumption rate of mozzarella cheese and limited low-sodium mozzarella sensory research, there is an apparent need for understanding how consumers perceive low-sodium mozzarella cheese rather than cheddar cheese or lower consumption cheeses.

\subsection{Sensory Evaluation}

\subsubsection{Traditional Sensory Testing}

The frequently used Sensory Evaluation Division of the Institute of Food Technologists (Anonymous, 1975) definition of sensory evaluation is: "a scientific discipline used to evoke, measure, analyze, and interpret reactions to those characteristics of foods and materials as they are perceived by the senses of sight, smell, taste, touch, and hearing."Sensory evaluation has been used for decades, across a variety of industries and by both government and private parties to gain knowledge of product acceptability, similarities, or differences by a target group. The importance of sensory evaluation appears to be generally agreed upon; however, the specific methods and techniques to employ are still debated (Stone et al., 2012). Although a variety of sensory testing techniques exist, a brief review of affective, discrimination, and descriptive sensory testing will be covered to outline each of these methods.

\subsubsection{Affective Testing}

Affective testing within sensory evaluation is typically employed amongst current or potential consumers to evaluate the "personal response" or liking to a product or product idea by way of measuring preference or acceptance (Meilgaard et al., 2007). The 
aim of affective testing is to gather information about a target population therefore screening for the appropriate subjects is essential; however, it is not expected that these subjects have been screened for sensory acuity or trained in sensory (Stone et al., 2012). The two most commonly used affective tests are: 1) the paired-comparison test and 2) the 9-point hedonic test. Other methods exist, however these are typically adaptations of the two mentioned tests. The paired-comparison test requires the subject to select the preferred product, either A or B; in some cases a "no preference" or "dislike-bothequally" may be selected as well. This test is very simple for subjects to understand and perform, and given the correct test wording and design, provides an overall result of preference between two products. The paired-comparison test does not provide any degree of difference between the two products, only if there is a preference or not (Meilgaard et al., 2007; Stone et al., 2012). If the objective of the test is to determine a measureable difference in acceptability then a 9-point hedonic test should be used. The 9point categorical scale typically wording is: "dislike extremely" as "1" to "like extremely" as "9" (Fig. 2.1).

\section{Example of 9-point Hedonic Scale}

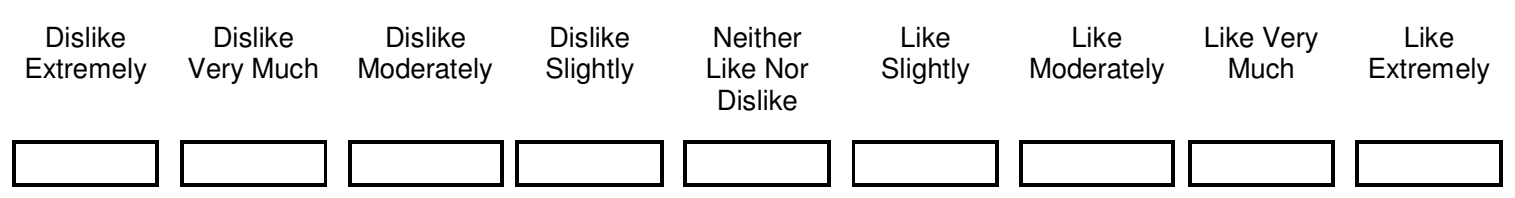

Fig. 2.1. Example of 9-Point Hedonic Scale. The 9-point hedonic scale can be used for scoring a variety of liking attributes.

This test is widely used because of the ease of implementation and understanding by untrained subjects during testing; however, some criticize the potential neutral category 
avoidance by subjects and lack of evidence for equality between categories (Stone et al., 2012).

\subsubsection{Discrimination Testing}

Discrimination testing has stricter requirements for subjects including: 1) being a product user, 2) frequent participation in sensory tests, 3) not have information about the project at hand, and 4) having a certain level of sensory acuity. Two frequently used discrimination tests are the duo-trio and triangle tests. The duo-trio test requires group of unknowns where the subject is tasked with selecting the product which is most similar to the reference sample and the chance probability is $p=1 / 2$ (Stone et al., 2012). The popular triangle test uses two different samples again; however, two cups contain the same product and one cup contains the other product, with possible order presentations being: ABB, BAA, AAB, BBA, ABA, and BAB (Meilgaard et al., 2007). Some sensory scientists suggest that the duo-trio test is statistically less effective compared to the triangle test because of the chance probability (Meilgaard et al., 2007). Others argue that although the chance probability of the triangle test is $p=0.333$, it is in fact not more statistically sensitive because "fewer correct scores required for statistical significance should not be confused with the totally separate issue of sensitivity" (Stone et al., 2012).

\subsubsection{Descriptive Analysis}

Descriptive sensory analysis is considered by some to be the most sophisticated and sensitive sensory testing tool (Murray et al., 2001). At least seven different descriptive sensory methods exist; however, only two of the most commonly used methods will be reviewed here: Quantitative Descriptive AnalysisTM (Stone et al., 1974) 
and the Spectrum ${ }^{\mathrm{TM}}$ method (Meilgaard et al., 1991). Descriptive analysis techniques employ the use of $8-15$ qualified subjects who conduct a complete sensory evaluation using quantitative descriptions of products (Stone et al., 2012; Meilgaard et al., 2007; Murray et al., 2001). Descriptive analysis participants are tasked with "taking into account all sensations that are perceived - visual, auditory, olfactory, kinesthetic, etc. when the product is evaluated" (Stone et al., 2012).

The Quantitative Descriptive Analysis ${ }^{\mathrm{TM}}$ (QDA) method was developed in the 1970's to help eliminate some perceived problems with the descriptive methods used at the time (Murray et al., 2001), specifically the lack of quantitative data and the use of “expert” panelists (Stone et al., 2012). The QDA ${ }^{\mathrm{TM}}$ method uses consumer language to reduce bias of words provided in a lexicon; subjects are selected based upon sensory acuity and being a consumer of the test product. Reference standards are rarely used during language development, scores are captured on an unstructured line scale, and the panel moderator does not contribute to product scoring in QDA ${ }^{\mathrm{TM}}$ (Murray et al., 2001). Subjects in QDA ${ }^{\mathrm{TM}}$ do go through training, approximately 10-15 hours (Murray et al., 2001); however, subjects test samples in at least triplicate to decrease variability within subjects and disregard any between subject variance (Stone et al., 2012).

The Spectrum ${ }^{\mathrm{TM}}$ method was also developed in the 1970's and uses extensive reference lists, and "specialize panel training and scaling procedures" (Murray et al., 2001). The Spectrum ${ }^{\mathrm{TM}}$ method carries out approximately 100 hours of panelist training prior to testing, and requires the panelists understand the basic physiology and psychology of sensory. This method uses line scales with specific product reference points along the range of the scale to decrease variability between panelists. The cost 
associated with the long training and number of reference samples can be quite high; however, a major perceived benefit of the Spectrum ${ }^{\mathrm{TM}}$ method is the expected total calibration of the panelists (Murray et al., 2001; Lawless \& Heymann, 1998).

The QDA ${ }^{\mathrm{TM}}$ and Spectrum ${ }^{\mathrm{TM}}$ methods are quite different; therefore, selection of the most appropriate method may depend on specific project objectives and parameters. Murray et al. (2001) argues that if a company needed descriptive analysis performed on a product which had been tested previously by the descriptive panel and a large budget existed, perhaps the Spectrum ${ }^{\mathrm{TM}}$ method would be best. If the company had a product which had not been previously evaluated by a descriptive analysis panel in the past and the project budget was more limited, then perhaps the QDA ${ }^{\mathrm{TM}}$ method would be the better choice.

\subsubsection{Current Sensory Evaluation of Low Sodium Cheeses}

Considering the expressed need for reducing sodium in foods, the number of studies involved in reducing sodium in dairy products, specifically cheese, is unsurprising. Studies investigating the sensory effects of sodium reduction in cheese have focused on a variety of different cheese products including: cheddar cheese (Grummer et al., 2012; Guinee, 2004; Fitzgerald \& Buckley, 1985), feta cheese (Katsiari et al., 1997), imitation cheese (El-Bakry et al., 2011), cottage cheese and cheese sauces (Drake et al., 2011). Approaches to reducing sodium typically focus on either production of cheese by reducing the amount of $\mathrm{NaCl}$ or by substituting $\mathrm{NaCl}$ (Cruz et al., 2011). 


\subsubsection{Decrease NaCl Concentration}

One method for reducing sodium in foods is simply to decrease the concentration of $\mathrm{NaCl}$ in the product (El-Bakry et al., 2011). Imitation cheeses are a complex system with rather high sodium content due to $\mathrm{NaCl}$ and emulsifying salts $(\mathrm{ES})$ which create the essential homogenous characteristic of the cheese product (El-Bakry et al., 2011). ElBakry et al. (2011) used the triangle test method (Meilgaard et al., 1991) to evaluate two $48 \%$ moisture imitation cheeses: 1) $1.5 \% \mathrm{NaCl}$ (standard) and 2) $0.75 \% \mathrm{NaCl}$ with 24 panelists (14 female); in a separate test, panelists performed a preference test with the same products $(1.5 \%$ and $0.75 \% \mathrm{NaCl}$ cheeses). The authors reported no significant difference between the samples based upon the triangle test results, but $83 \%$ of panelists indicated a preference; amongst those with a preference, $56 \%$ of panelists preferred the reduced $\mathrm{NaCl}$ cheeses and $44 \%$ preferred the control (El-Bakry et al., 2011). The panelist qualification was not mentioned in the study; however, if panelists were not imitation cheese consumers it is possible that a true difference between the two samples may not have been detected in the triangle test due to low familiarity with the product category. Considering the significant preference for the lower sodium product amongst this small population, suggests reducing $\mathrm{NaCl}$ an appropriate amount could yield an acceptable product.

Drake et al. (2011) studied the effects of lowering sodium in both cottage cheese and cheese sauce products by way of sodium reduction. Variables included: 1) no reduction, 2) $4 \%$ reduction, 3) $8 \%$ reduction, and 4) $12 \%$ reduction and testing methods included: 1) just-noticeable difference (JND) threshold testing (lowest concentration that would cause a saltiness sensation) and 2) consumer testing to evaluate the products 
(Drake et al., 2011). The authors concluded that small reductions (2\% to 5\%) where not noticed by consumers and the "no reduction", "4\% reduction", and "8\% reduction" were not significantly different for overall liking, appearance liking, and salty intensity; all samples tested were not significantly different $(\mathrm{P}<0.05)$ for flavor liking (Drake et al., 2011). A main sodium reduction goal for several companies is to decrease sodium in foods by $25 \%$, thus this study suggests a $25 \%$ reduction in sodium would likely be detected and accepted significantly less than the traditional product by consumers.

\subsubsection{Substitution of $\mathrm{NaCl}$}

Substitution of $\mathrm{NaCl}$ with potassium chloride $(\mathrm{KCl})$ is a frequently used method to lower the sodium content in foods by $25 \%$ without the loss of palatability (Cruz et al., 2011); however, substitution of $\mathrm{KCl}$ for $\mathrm{NaCl}$ must be done with care considering $\mathrm{KCl}$ can leave a metallic, sour dairy and/or bitter note in cheeses (Cruz et al., 2011; Grummer et al., 2012). Grummer et al. (2012) investigated the use of $\mathrm{NaCl}$ in cheddar cheese with the following variables: sea salt, $\mathrm{KCl}$, modified $\mathrm{KCl}, \mathrm{MgCl}_{2}, \mathrm{CaCl}_{2}$, and sea salt with: $\mathrm{KCl}$, modified $\mathrm{KCl}$, and $\mathrm{MgCl}_{2}$. The descriptive analysis results indicated the cheddar cheeses with $\mathrm{NaCl} \& \mathrm{KCl}$ and $\mathrm{NaCl} \&$ modified $\mathrm{KCl}$ were positioned the closest to the $\mathrm{NaCl}$ standard on a Principle Component Analysis (PCA). Grummer et al., (2012) suggested the relative proximity of these variables to the control indicated they had similar sensory attributes. Further investigation of these results found the control and $\mathrm{NaCl} \&$ modified $\mathrm{KCl}$ did differ significantly in overall flavor, but all other flavor attributes did not differ significantly; no consumer acceptability or hedonic data was collected in the study. 
When partial substitution of $\mathrm{NaCl}$ by $\mathrm{KCl}$ was tested in feta cheese with the following variables: 1) control, 2) $3: 1 \mathrm{NaCl}: \mathrm{KCl}$, and 3) $1: 1 \mathrm{NaCl}: \mathrm{KCl}$, no significant difference in appearance, body and texture, flavor, and "total score" was found (Katsiari et al., 1997). However, the 5 member panel rated the samples on levels of "quality" rather than the conventional intensity scale; additionally, neither the level of training nor qualification for being a panel member was mentioned. Katsiari et al., (1997) concluded that the cheeses did not differ significantly from the control while having a $25 \%$ to $50 \%$ lower sodium concentration, a $1 \mathrm{NaCl}$ : $1 \mathrm{KCl}$ ratio was suggested based on their results and nutrition recommendations.

Ayyash and Shah (2011) evaluated the chemical composition and functional properties of partially substituted $\mathrm{KCl}$ mozzarella cheese; however, no sensory or texture analysis was performed on the samples. Two functional properties which are somewhat related to sensory were evaluated: meltability and browning. The $1 \mathrm{NaCl}: 1 \mathrm{KCl}$, and $1 \mathrm{NaCl}: 3 \mathrm{KCl}$ salted samples were higher in meltability and browning compared to the $\mathrm{NaCl}$ only control (Ayyash \& Shah, 2011). Although this information is a positive step toward understanding the role of $\mathrm{KCl}$ in mozzarella cheese, additional information is needed about consumer acceptability, descriptive and texture analysis of $\mathrm{KCl}$ substituted and $\mathrm{NaCl}$ reduced mozzarella cheese. Based upon studies which have observed the effects of $\mathrm{KCl}$ partial substitution in different cheeses, a suggested ratio close to $1 \mathrm{NaCl}: 1 \mathrm{KCl}$ appears to be the most effective from both a functional and sensory perspective (Ayyash \& Shah, 2011; Cruz et al., 2011; Katsiari et al., 1997); however, other studies have suggested a $70 \% \mathrm{NaCl}$ to $30 \% \mathrm{KCl}$ may be more appropriate (Guinee, 2004; Guinee \& O’Kennedy, 2007), clearly further research is needed. Cruz et al. (2011) 
stated that a group of United Kingdom specialists suggested a sodium reduction of 10 to $25 \%$ could not be detected by consumers; however, other studies have suggested otherwise (Drake et al., 2011; Johnson et al., 2009). Substitution of $\mathrm{NaCl}$ with $\mathrm{KCl}$ has been studied in a variety of different cheeses, most of which have strong or complex flavors (i.e. cheddar and feta); however, mozzarella cheese is known for being a blander cheese, in which case the $\mathrm{KCl}$ substitution may be more obvious, thus additional research in this area is needed. Sodium reduction in cheese is an obviously challenging goal, but by assessing other aspects of the cheese consumption experience, researchers may gain a clearer understanding of how to address this difficult objective.

\subsection{Emotions}

\subsubsection{Why Evaluate Emotions?}

Several sensory researchers have suggested the need for additional information above and beyond what basic hedonics provides with the goal of better understanding consumer food choice behavior (King \& Meiselman, 2010; Macht, 2008; Macht \& Simons, 2000; Seo et al., 2009; Thomson et al., 2010). Thomson et al. (2010) argued that with increased exposure to a product, consumers develop a conceptual association to that specific product. The authors defined these conceptualizations as "constructions created in the mind that allow us to interpret, understand and otherwise assign meaning to what we experience." Eventually, the authors stated that conceptualization, despite being "infinitely diverse"; could be categorized into three key concepts: functional, emotional, and abstract conceptualizations (Fig. 2.2) and are described as follows:

- Functional: the product meets the basic functional needs required of the product. 
- Emotional: elicits certain emotional responses (i.e. happy, sad, calming, etc.).

- Abstract: something which cannot really be measured (i.e. sophisticated, trustworthy, etc.).

The authors argued that the abstract conceptualizations were precursors to either emotional and/or function conceptualizations, suggesting ultimately that all conceptualizations fall into the category of either emotional or functional conceptualization (Thomson et al., 2010).

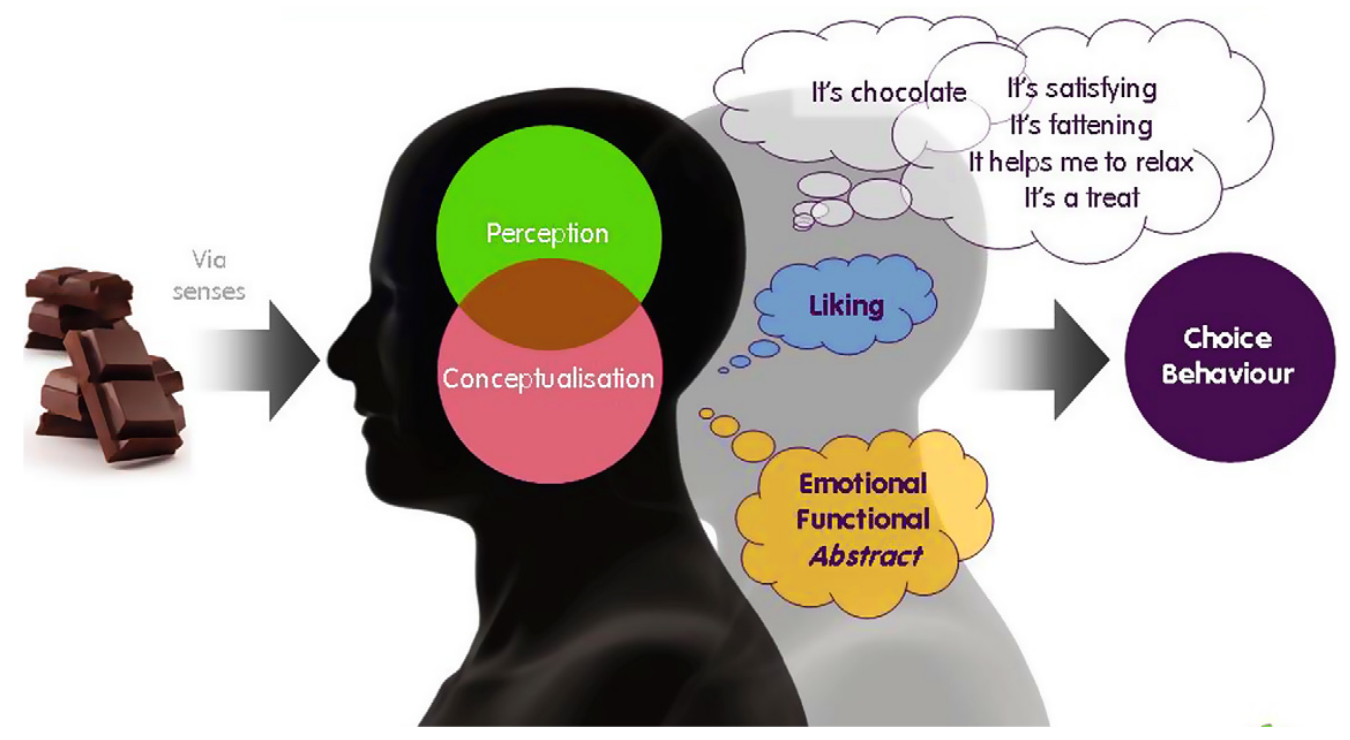

Fig. 2.2. Consumer Perception and Conceptualization of Products. Consumer familiarity to a product can develop conceptualizations which are comprised of emotional, functional, and abstract aspects (Thomson et al., 2010).

Measuring emotional affects or the "emotional consequence" of products could provide additional insight into consumer choice behavior which may better create a successful product and brand (Thomson et al., 2010). Many other researchers have recognized the important relationship between emotions, product choice and purchase, yielding a variety of different emotion measurement testing methods (Desmet \& Schifferstein, 2008; Gard 
\& Kring, 2007; King \& Meiselman, 2010; Macht \& Simons, 2000; McNair et al., 1971; Thomson et al., 2010).

Human eating behavior, which is typically influenced by a food, bodily, social and physical environment cues, is generally accepted to also be affected by and related to emotions (Desmet \& Shifferstein, 2008). Although traditional sensory evaluation of food has focused on the measuring of basic tastes, it is understood that consumers often purchase food products "based on emotional response triggered by the products" (Seo et al., 2009). The use of emotion testing alone or in addition to traditional consumer sensory evaluation is gaining popularity within the industry (King \& Meiselman, 2010). Increase in the use of emotion testing is likely due to the hypothesis that consumer purchase behavior can be driven by emotional responses which are elicited by the chosen product, rather than just the basic taste profile of the product (Seo et al., 2009). Due to the promising future of emotions in sensory evaluation, a variety of different emotion testing methods have been developed (i.e. Croy et al., 2011; King \& Meiselman, 2010; Thomson et al., 2010; Seo et al., 2009; Gard \& Kring, 2007); these emotion tests seek to accurately capture the consumer's emotional relationship with food and purchasing intent. Several current emotion testing methods are well thought out and privy to the psychology of emotions; however, in order to critically evaluate these methods in this review, decision making, reward mechanisms, specific stimuli, and emotional processing will be assessed as they likely play a significant role of human emotional responses. 


\subsubsection{Decision Making and Reward Mechanisms}

The purpose of this section is to provide a brief overview of brain regions which are associated with decision making, reward-based decision making, and taste reward mechanisms to ultimately shed light on key brain systems for understanding the consumer psychology of decision making and emotions. As mentioned previously consumers are thought to choose products based upon information beyond product liking; by understanding the decision making process and reward mechanisms, emotion testing researchers may create a method which better addresses the nature of the emotion and product interaction.

Decision making is a process of evaluating available information to determine the best possible option (Glimcher, 2001; Brown \& Ridderinkhof, 2009). The areas of the brain thought to be most associated with decision making are: the striatal reward circuitry, the orbitofrontal cortex (OFC), the medial frontal cortex (MFC), and the insula and the dopamine neurotransmitter system (Brown \& Ridderinkhof, 2009). The striatal reward circuitry, as the name implies, is strongly related to reward mechanisms with the ventral striatum associated with reward magnitude while the dorsal striatum represents the reward probability (Brown \& Ridderinkhof, 2009). The OFC and striatal dopamine system are well recognized as being involved in reward magnitude and both systems are thought to be important contributors to reward-based decision making (Brown \& Ridderinkhof, 2009; Cromwell \& Schultz, 2003). Additionally, a variety of different brain circuits are thought to be associated with "emotional modulation of decision making" these areas include: the OFC, the anterior cingulate cortex (ACC), the amygdala, and the insula (Brown \& Ridderinkhof, 2009). Although emotional reasoning 
was not included in early decision making theories, it is currently thought to be important in the assessment of reward outcomes (Brown \& Ridderinkhof, 2009), which agrees with Thomson et al.'s (2010) concept for consumer decision making. Lastly, areas in the brain thought to be associated with rewards include: the midbrain, striatum, OFC, and amygdala. The OFC and amygdala appear to be pivotal brain areas for decision making and reward processing; therefore they will be explored further and eventually connected to salty taste rewards. Fig. 2.3 outlines the key regions of the brain for decision making, although not all components are included.

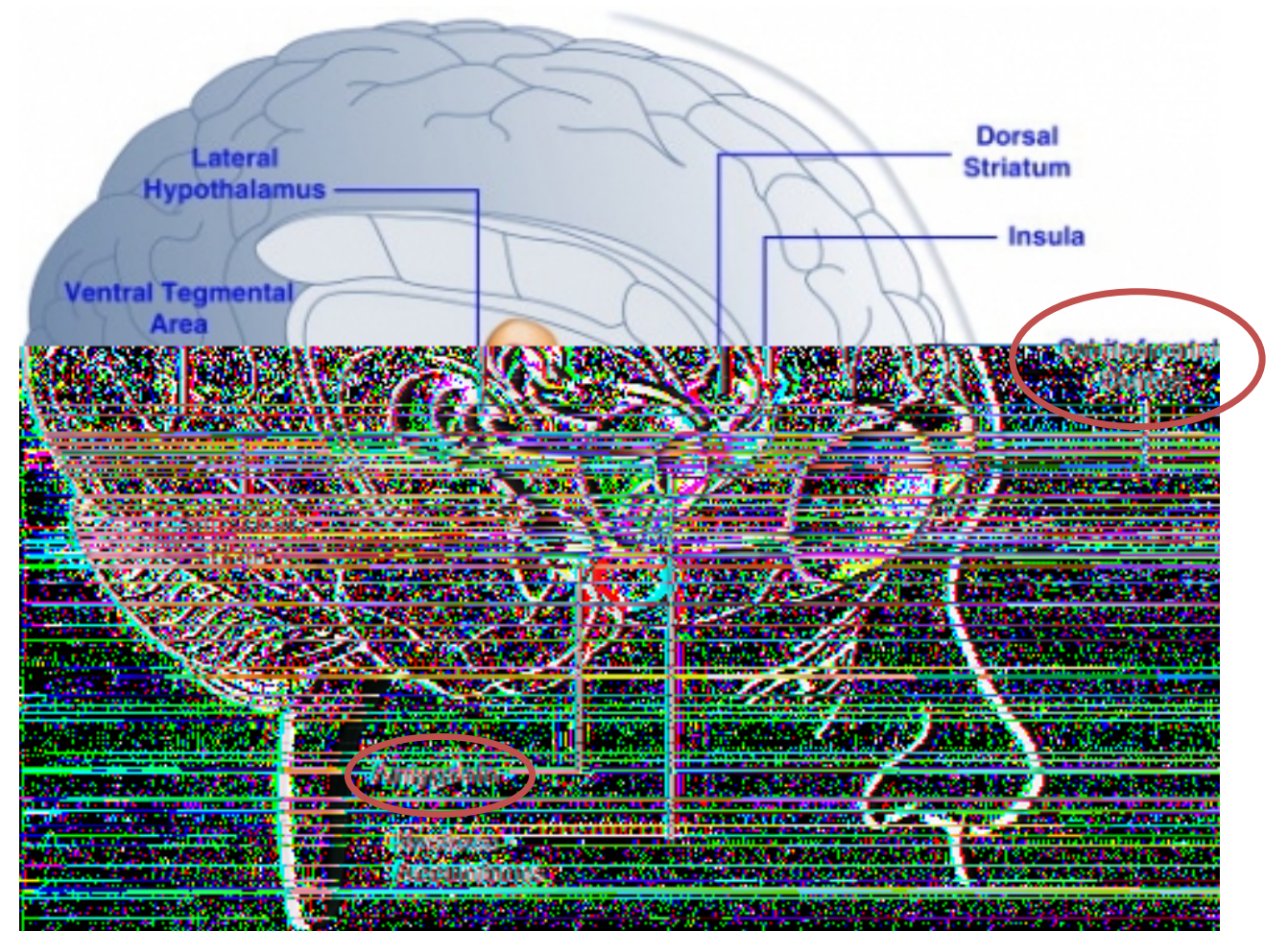

Fig. 2.3. Decision Making and Reward Mechanism Areas of the Brain. The key areas of the brain involved in decision making and reward mechanisms, specifically the amygdala and orbitofrontal cortex (OFC) (Kenny, 2011).

The OFC houses the "affective value" of unlearned rewards such as taste, touch, texture, and facial expression. It eventually associates additional stimuli with these unlearned rewards to anticipate reward values for visual, auditory and abstract stimuli; 
thus producing the call to action and ultimately playing a key role in emotions (Rolls \& Grabenhorst, 2008). O'Doherty et al. (2002) investigated the areas of the brain involved with reward anticipation and receipt of reward and found the OFC was involved with reward anticipation due to neuron activity by visual cues which predicted the delivery or expectation of a reward. The OFC and amygdala have been investigated during studies of sensory-specific satiety, where subjects are provided two food stimuli and fed until satiated with one of the two foods, this eventually leads to a decrease in the reward value of the consumed food which can be seen in the OFC and amygdala with reward value decreasing for the consumed food, but not for the uneaten food (Kringelbach, 2009). One explanation for the significant change in eating behavior in recent years could be due to malfunctions in these satiation mechanisms; however, lesions in the OFC are currently not associated with obesity (Kringelbach, 2009).

In addition to the significant role of the OFC and amygdala in general reward mechanisms, their importance in salt specific rewards has also been investigated; the OFC was found to be a key region responding to both anticipation of salt and receipt of salty taste (O’Doherty et al., 2002). This study characterized salt as a negative reward and glucose as a positive reward, therefore the areas of the OFC which activated upon receipt of the salty taste were recognized as responding to unpleasant stimuli. The same study determined that a part of the amygdala responding to "primary reinforcers" specifically unpleasant stimuli including taste, odor, and flavors (O’Doherty et al., 2002), showed a heightened response to glucose anticipation compared to salt, with no observed responses in the amygdala to anticipation or receipt of salt. A salt concentration being too low to cause an aversive taste during testing could explain the lack of activation in the amygdala 
with salty stimuli (O'Doherty et al., 2002). Another study which explored reward behavior in rats found that even at the most preferred concentration, $\mathrm{NaCl}$ does not have the same hedonic impact as sugar, even when salt deprived; rats will not undergo brain agitation for salt but will for sugar (McCaughey \& Scott, 1998). Although the reinforcement for salt may be more subtle than that of sugar, a reward for consumption does exist (McCaughey \& Scott, 1998). The above studies explored the reward mechanisms of salt under two extreme conditions: 1) during salt depletion in the body and 2) salt concentration as an irritant. Little information is known about salt rewards at typically consumed levels in those with healthy to high levels of $\mathrm{NaCl}$ in the body, future research should explore this relationship.

\subsubsection{Brain Relationship between Emotion, Taste, Texture, and Images}

Different brain regions and mechanisms for rewards and decision-making were explored in the previous section; this section will investigate the brain regions and processes associated with emotion, taste, texture, and images. The relationships between these stimuli and emotion need to be understood in order to develop a comprehensive emotion evaluation testing method in the context of eating.

\subsubsection{Emotion}

The amygdala has been thought to play a role in emotional significance regulation of certain events (Martin-Soelch et al., 2007). The amygdala has also been associated with fear, aggression, and rage and interactions with the hippocampus for transcription and storage of emotional memories (O'Doherty et al., 2002; Freberg, 2009; Sander, 2012). However, Sander (2012) suggested little empirical evidence exists to make the 
overly simplified relationship between the amygdala and fear. Alternatively, he proposed the amygdala is capable of taking broader information into consideration including an individual's immediate concerns such as "goals, needs, and values" and thus suggested the amygdala is crucial to affective relevance. Sander (2012) also argued that relevance detection occurs before any mental representation of bodily responses because relevance detection is involved with eliciting emotional responses, which includes the "generalization of bodily sensations." The general role of the amygdala is to identify both positive and negative experiences which are subjectively evaluated based upon the individual's current needs, goals, and values; ultimately being a key driver in emotional and motivational assessment (Sander, 2012).

In addition to the amygdala's significant role in emotional responses, the OFC is thought to have major contributions to emotional assessment (Rolls, 1999; Rolls \& Grabenhorst, 2008). Emotions are reactions to reinforcing or reward stimuli and the OFC contains major cortical representation of taste and texture (among other sensations) which are primary reinforcers, thus suggesting a role of the OFC with emotions (Rolls, 1999; Rolls \& Grabenhorst, 2008). Rolls and Grabenhorst's (2008) general theory about emotions is similar to that of Sander (2012) suggesting emotions are periods prompted by goals or reinforcers. However, Rolls and Grabenhorst (2008) also have suggested the tool by which the reinforcer is presented will modify the emotional outcome; stating an individual's emotional state will differ depending on which reward (food, monetary, social, etc.) was or was not presented. Not only is the OFC involved in emotional responses through primary reinforcers, but physical damage to the OFC has been shown 
to impair an individual's "emotional behavior and subjective emotional states" (Rolls et al., 1994).

\subsubsection{Taste and Texture}

In primates, taste neurons can be found in the OFC, hypothalamus, ventral striatum, and the amygdala, in addition to these locations the primary taste cortex contains neurons sensitive to $\mathrm{NaCl}$, glucose, $\mathrm{HCl}$, and quinine (Rolls, 1997). The OFC contains certain neurons which only respond to taste, others which respond to different oral stimuli including viscosity and texture, and the use of fMRIs has demonstrated activation in the medial region of the OFC by taste, texture, and olfactory stimuli (Rolls \& Grabenhorst, 2008). Flavor is defined as the combination of taste, aroma, and chemical sensations (Meilgaard et al., 2007). The merging of taste and aroma is thought to occur in the OFC, thus playing a significant role in the pleasantness affect and reward mechanisms in the OFC (Rolls \& Grabenhorst, 2008). Certain tastes or aromas acting alone may be perceived as unpleasant, but the combination of taste and aroma produces a pleasurable sensation and positive reward value in the OFC. Neurons exist in the OFC which respond to textures in the mouth including varying viscosities and fatty mouthfeel and fat in mouth is perceived as pleasant when hungry; however, fatty food eating induced satiety can decrease the OFC neuron responses to the texture of fat in mouth (Rolls \& Grabenhorst, 2008).

In addition to the importance of the OFC, the amygdala is involved in feeding control due to its sensitivity to taste inputs and responses from other brain regions including the primary taste cortex which sends gustatory information to nuclei in three 
regions of the amygdala (Scott et al., 1993). The amygdala has been found to be specifically important for sodium intake, with lesions to the central amygdala causing decreased sodium intake (Li et al., 2012). Li et al. (2012) found when the central nucleus of the amygdala $(\mathrm{CeA})$ was damaged in rats, there was a significant increase in salt $(\mathrm{NaCl})$ sensitivity; suggesting this brain region is involved in the interpretation of salty taste intensity. Li et al. (2012) also found involvement of the CeA in not only regulating salty taste intensity, but also regulation of sodium consumption. Scott et al. (1993) found $63 \%$ of "taste-responsive cells" in the amygdala gave their highest responses to either glucose or $\mathrm{NaCl}$, suggesting the sensitivity to these primary tastes compared to that of sour and bitter flavors. Taste, specifically salty taste, appears to play a significant role in brain regions (the OFC and amygdala) which also interpret reward values, emotions, and decision-making. By decreasing salt in food and beverage products, there may be a physiological consequence prompting the negative hedonic response measured by traditional sensory methods that could be explained with concurrent emotion testing.

\subsubsection{Images}

General interpretation of the brain has suggested the left hemisphere is associated more with language, mathematics, and logical processing, while the right hemisphere is associated with music, intuition, and art; however, this is known to be an over simplification of the true processes (Freberg, 2009). Psychologists have generally understood common links which exist between emotions and mental imagery rather than other types of processes; however, little empirical evidence has been able to validate this theory (Holmes et al., 2008). Holmes et al. (2008) argued that emotions and images are linked for the following three reasons: 1) basic emotion processes developed early on in 
the brain, prior to language, and are more sensitive to "sensory-perceptual" information suggesting images may elicit emotions more easily than language-based concepts, 2) memories from imagined events can be confused with actual event memories, this type of confusion is more likely when imaged based techniques are used rather than verbal techniques, and 3) personal memories are mostly stored as images including the emotional association to those memories; suggesting images may be helpful in remembering certain memories with their corresponding emotion.

These theories for linking emotions and mental imagery are in accordance with the Zaltman Metaphor Elicitation Technique (ZMET), a well-established and validated consumer metaphor-based research method. The first three principles of the ZMET method are: 1) most communication is nonverbal, 2) thought occurs as images, and 3) metaphors are essential units of thought; the method suggests the importance of researching beyond the linguistic language with consumers and focusing on understanding their thoughts, emotions, and memories by way of images. The ZMET method suggests the use of images allows researchers to get closer to how thoughts are processed and thus a clearer idea of how the consumer really thinks (Zaltman \& Coulter, 1995).

Holmes et al. (2008) evaluated the use of images versus words for emotion testing where subjects were asked to perform a task with either verbal or visual instructions, a "memory for words" taste, an anxiety questionnaire for the tasks, and a subjective experience questionnaire regarding the performed tasks. Results from this study found the image instructions, compared to verbal instructions, elicited: 1) increased anxiety after viewing negative pictures and 2 ) decreased anxiety after viewing the neutral pictures. The 
magnified emotional response in the image testing suggests increased "evaluative learning" and imagery instructions elicited more emotional valence than verbal instructions. Holmes et al. (2008) ultimately argued that the study suggests "imagery has causal effects on emotion."

In addition to these arguments by Holmes et al. (2008), Rolls and Grabenhorst (2008) suggest both visual and emotional processing occur in the OFC and Rolls et al. (2005) found a group of neurons in the primate OFC which respond to only new visual stimuli. The specific purpose of these neurons is unknown; however, activation of this area of the OFC in humans has been associated with the encoding of memories of new visual stimuli (Rolls et al., 2005).

Overall there appears to be findings which suggest a relationship between the brain processing areas involved with emotions, taste and texture, and images. Fig. 2.4 provides the processes by which different senses are processed in the brain, with the OFC and amygdala appearing to be main centers for all the indicated senses. Each sensation follows a series of pathways before arriving at the OFC and amygdala which interpret the sensation information and relay it to other areas of the brain for additional interpretation and reaction to the specific sensation (Rolls \& Grabenhorst, 2008). Additionally, many of these brain regions share similarities with decision-making and reward mechanisms in the brain. This overview of brain regions and processes has brought to light important information and will be used as a criterion for evaluating current emotion testing methods. 


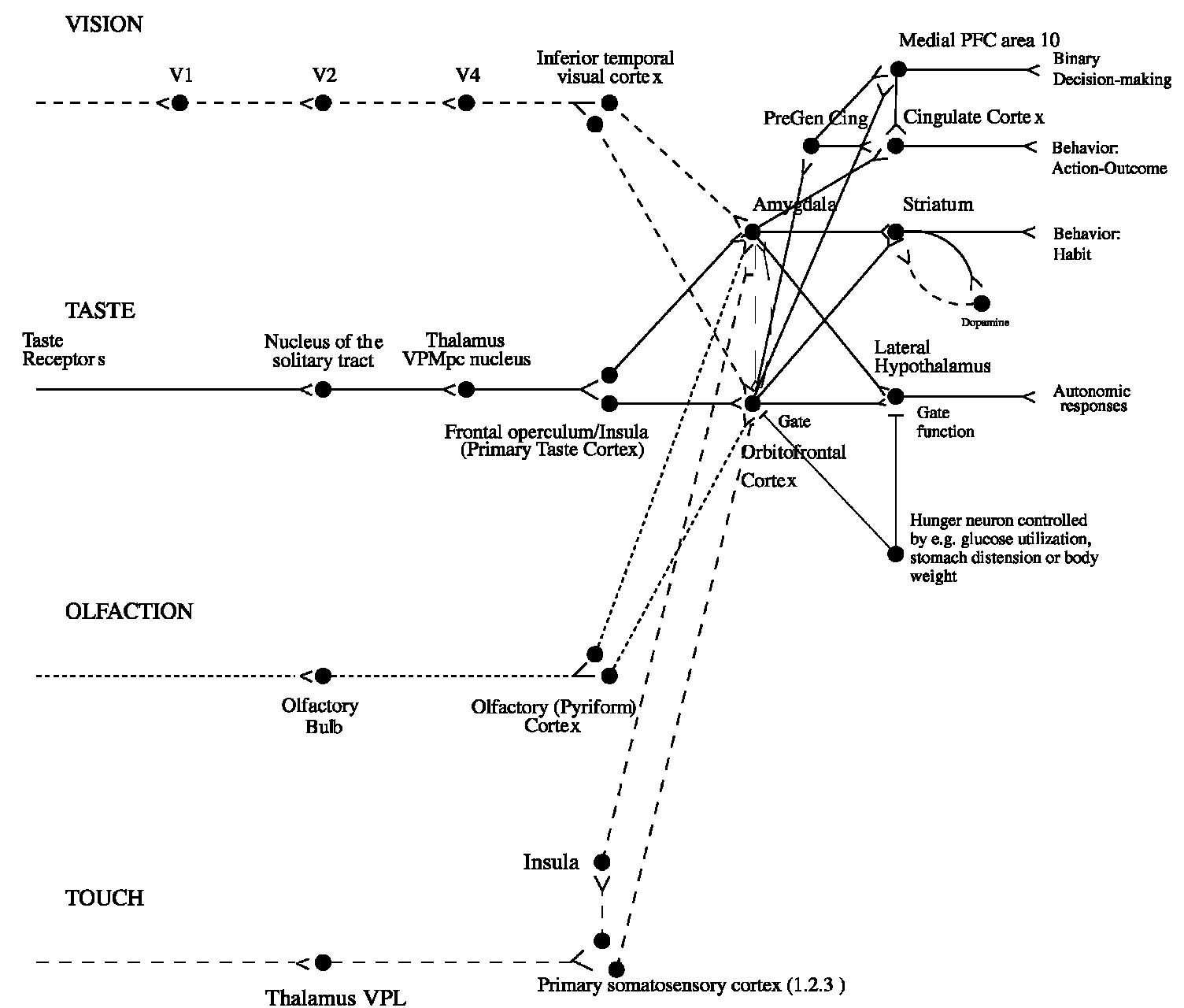

Fig. 2.4. Sensation Convergence Pathways in the Brain. Diagram showing the convergence of visual, taste, olfaction, and touch pathways in the brain; clear importance of the amygdala and OFC can be seen (Rolls \& Grabenhorst, 2008).

\subsubsection{Current Emotion Testing Methods}

Understanding consumer's emotional responses to products has become a major focus within sensory and consumer science (i.e. King et al., 2010; Macht, 2008; Thomson et al., 2010). Although a variety of different emotion testing methods have been developed, few appear to have been critically tested as a validation process and are oftentimes hybrids of other methods. This review will investigate emotion testing methods from King and Meiselman (2010), Thomson et al. (2010), Desmet et al. (2000), and Croy et al. (2011) as these are unique, published, and relatively recent methods. 


\subsubsection{EsSense Profile ${ }^{\mathrm{TM}}$ Method}

King and Meiselman (2010) developed the EsSense Profile TM emotion testing method in 2008 with the goal of understanding the consumer's emotional response to a product rather than the brand and to measure emotions rather than moods which have been evaluated in psychology for decades (McNair et al., 1971; Zuckerman \& Lubin, 1965). King and Meiselman (2010) conducted a series of tests including central location tests (CLT), home use tests (HUT), focus groups, and internet surveys to determine the source of emotion terms, identify emotion terms which were easily understood by consumers, select emotion terms which were relevant to consumer's eating experiences with liked and disliked products, and develop a questionnaire scaling system. This method was developed for commercial testing use and although it provides additional information beyond acceptability, the emotion scores can still be compared to typical consumer testing scores such as hedonics and acceptability (King \& Meiselman, 2010). The EsSense Profile ${ }^{\mathrm{TM}}$ method contains 39 mostly positive emotions (Table 2.2). The authors argue a large number of emotions are necessary in order to capture as many of the emotional responses and differences that may exist between the products; additionally, product users tend to have a positive relationship toward the product and suggest negative emotions are not as needed in testing as positive emotions (King \& Meiselman, 2010). The authors suggest methods which use a small list of emotions ("small" was not quantified) risk missing valuable information, specifically when a large proportion of negative emotions are used. Product users versus non-users need to be specified, as these groups have shown to differ in their emotional responses to the same product. When comparing the use of a "checklist" questionnaire versus a scaling questionnaire, the 
authors found the checklist/choose-all-that-apply (CATA) format to be effective at distinguishing different flavor profile products, while the scaling questionnaire was effective at determining different flavors of the same product. Lastly, the authors suggest the EsSense Profile ${ }^{\mathrm{TM}}$ method is most effective when tested in a CLT or internet survey format and can provide a common emotion language which can be used to facilitate communication about emotions between the sensory science, marketing, and product development fields (King \& Meiselman, 2010).

Table 2.2. EsSense Profile ${ }^{\mathrm{TM}}$ List of Emotions. List of the 39 emotions selected for use in the EsSense Profile ${ }^{\mathrm{TM}}$ method (King \& Meiselman, 2010).

\begin{tabular}{|l|l|l|}
\hline \multicolumn{2}{|c|}{ EsSense Profile } \\
\hline \multicolumn{1}{|c|}{ TM List of Emotions } \\
\hline$\square$ Active & $\square$ Glad & $\square$ Pleasant \\
\hline$\square$ Adventurous & $\square$ Good & $\square$ Polite \\
\hline$\square$ Affectionate & $\square$ Good-natured & $\square$ Quiet \\
\hline$\square$ Aggressive & $\square$ Guilty & $\square$ Satisfied \\
\hline$\square$ Bored & $\square$ Happy & $\square$ Secure \\
\hline$\square$ Calm & $\square$ Interested & $\square$ Steady \\
\hline$\square$ Daring & $\square$ Joyful & $\square$ Tame \\
\hline$\square$ Disgusted & $\square$ Loving & $\square$ Tender \\
\hline$\square$ Eager & $\square$ Merry & $\square$ Understanding \\
\hline$\square$ Energetic & $\square$ Mild & $\square$ Warm \\
\hline$\square$ Enthusiastic & $\square$ Nostalgic & $\square$ Whole \\
\hline$\square$ Free & $\square$ Peaceful & $\square$ Wild \\
\hline$\square$ Friendly & $\square$ Pleased & $\square$ Worried \\
\hline
\end{tabular}

Considering the method was recently published in 2010, little published critiques of this method exist; however, it has been suggested the EsSense Profile ${ }^{\mathrm{TM}}$ method consists of emotion words which are not relative or applicable to certain products and consumers have found some words to be ambiguous (Jaeger, 2012). The EsSense Profile ${ }^{\mathrm{TM}}$ method has also been criticized for not taking cultural differences into account as translating the current emotion words often do not translate correctly (Spinelli, 2012), also not taking into account any aids to help easily elicit emotions, and its limited number of negative emotions. The popular and heavily used hedonic 9-point scale has been studied and evaluated for decades and the importance of a balanced scale has been noted and reviewed (Lim, 2011) and the asymmetry in emotion testing has been criticized 
(Schifferstein \& Desmet, 2010); perhaps the EsSense Profile ${ }^{\mathrm{TM}}$ method could look into creating a more balanced emotion list to better evaluated the eating experience. The EsSense Profile ${ }^{\mathrm{TM}}$ does appear to provide a simple and straightforward method for evaluating emotional responses; however, other methods may help decrease the limitations found in the current EsSense Profile ${ }^{\mathrm{TM}}$ method.

\subsubsection{Best-Worst Scaling}

Thomson et al. (2010) took a different approach when developing an emotion method with the primary call to action being to go beyond the "obvious, apparently intuitive and otherwise associated with immediate liking..." in order to capture more intrinsic motivations and conceptualizations which influence choice. The authors state that although words provide richness to language and an ease for emotion measurement, their use in emotion research has been criticized because it is wrongly assumed to require a type of measurement scale. The use of scaling leads subjects to think about the meaning of the words which involves the use of cognitive, rational thinking, while the objective of the test is to instead capture information about emotions and choice which maybe more non-cognitive or irrationally focused. Additionally, when words are used for scaling, subjects often focus on the literal meaning of the words, when in fact the metaphoric meaning often provides the fullness and complexity behind the word. Thomson et al. (2010) suggested the use of best-worst scaling (Jaeger et al., 2008) because it provides the opportunity to use words (ideal for researchers) without requiring "external measurement scales." The general objective of this method is to provide the subject with the test product and four or five words at a time from a larger list of terms, and the subject simply needs to decide which of the words provided s/he feels is the most and the 
least closely related to their experience with the current test product; this is repeated with different groups of four or five words from the larger list of terms. Upon completion, researchers are able to map the most and least relevant terms for the given product (Fig. 2.5) (Thomson et al., 2010).

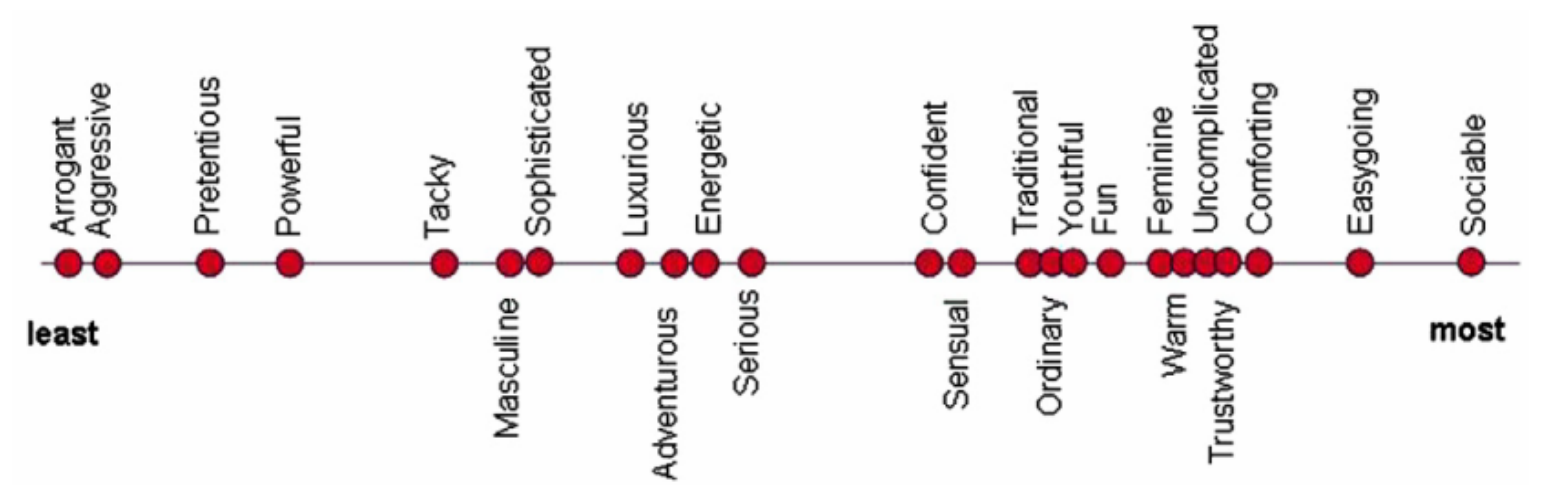

Fig. 2.5. Example of Best-Worst Scaling. An example of a best-worst scale used for assessing chocolates (Thomson et al., 2010).

The terms for the list are carefully selected based upon focus groups and the specific product being tested; typically 16-30 words are used for a given product (Table 2.3) with 20-30 specific product users. 
Table 2.3. Best-Worst Scaling List of Emotions. List of specific terms selected for testing dark chocolate with the best-worst scaling method (Thomson et al., 2010).

\section{Best-Worst List of Emotions}

Adventurous
Arrogant
Confident
Energetic
Fun
Masculine
Powerful
Sensual
Sociable
Tacky
Trustworthy
Warm

Aggressive Comforting

Easygoing

Feminine

Luxurious

Ordinary

Pretentious

Serious

Sophisticated

Traditional

Uncomplicated

Youthful

Although this method appears to be relatively simple to implement, strong statistics by way of multinomial logit (MNL) models or marginal MNL for determining the probability a subject would select a certain best-worst pair compared to another for a given product; also for comparing best-worst scaling scores and linking them with hedonic and descriptive analysis scores which use different measurement scales (Thomson et al., 2010). The best-worst scaling method can be implemented by researchers in pursuit of understanding consumer conceptualizations and non-cognitive responses to branded and un-branded products (Thomson et al., 2010).

Thomson et al. (2010) suggested the use of best-worst scaling for conceptualization, which as defined earlier consists of emotions of products; this method does appear to clarify the source of these experiences better than the EsSense Profile ${ }^{\mathrm{TM}}$ method. A main argument against the EsSense Profile ${ }^{\mathrm{TM}}$ method is potential subject misinterpretation of the words, despite the different scaling and implementation of this best-worst scaling method compared to the EsSense Profile ${ }^{\mathrm{TM}}$ method, the best-worst scaling does still in fact use words to convey concepts, ideas, and emotions to subjects. If the use of words is recognized to cause ambiguity, lack of cultural diversity, and general 
misinterpretation of the terms, why are words still being implemented in emotion and conceptualization testing?

\subsubsection{Product Emotion Measure (PrEmo)}

Desmet et al. (2000) agreed with the significance of measuring consumer emotional responses to products and the method of measurement must be capable of detecting emotions quickly, at low intensities, and across different cultures. The authors suggested psychophysical instruments for measuring emotion are not effective because of their lack of specificity and intensity for each emotion; therefore self -reported emotion methods are more effective. Additionally, the self-reported emotion method should be non-verbally based because: 1) consumers may not be capable of adequately describing how they feel and emotions elicited by certain stimuli can be difficult to characterize, 2) asking consumers to describe their current emotions requires cognitive engagement which may affect the measurement, and 3) non-verbal representation of emotion may translate better between different cultures (Desmet et al., 2000). The authors developed a method (PrEmo) for evaluating emotions with consideration of the above criteria, but the basis for this method was to relate emotions to the appearance of a static product. The PrEmo is a self-reported method with a series of 18 cartoon illustrations (Fig. 2.6) and each illustration animates an emotion for one second. The authors selected nine positive emotions: enthusiastic, inspired, desiring, appreciative, pleasantly surprised, attracted, content, fascinated, and softened, and nine negative emotions: disgusted, indignant, contempt, aversive, disappointed, dissatisfied, bored, disillusioned, and vulnerable; these emotions were selected based upon emotions which can be elicited by the product 
appearance, not from buying or using the product (Desmet et al., 2000). Note: no neutral emotions were included.

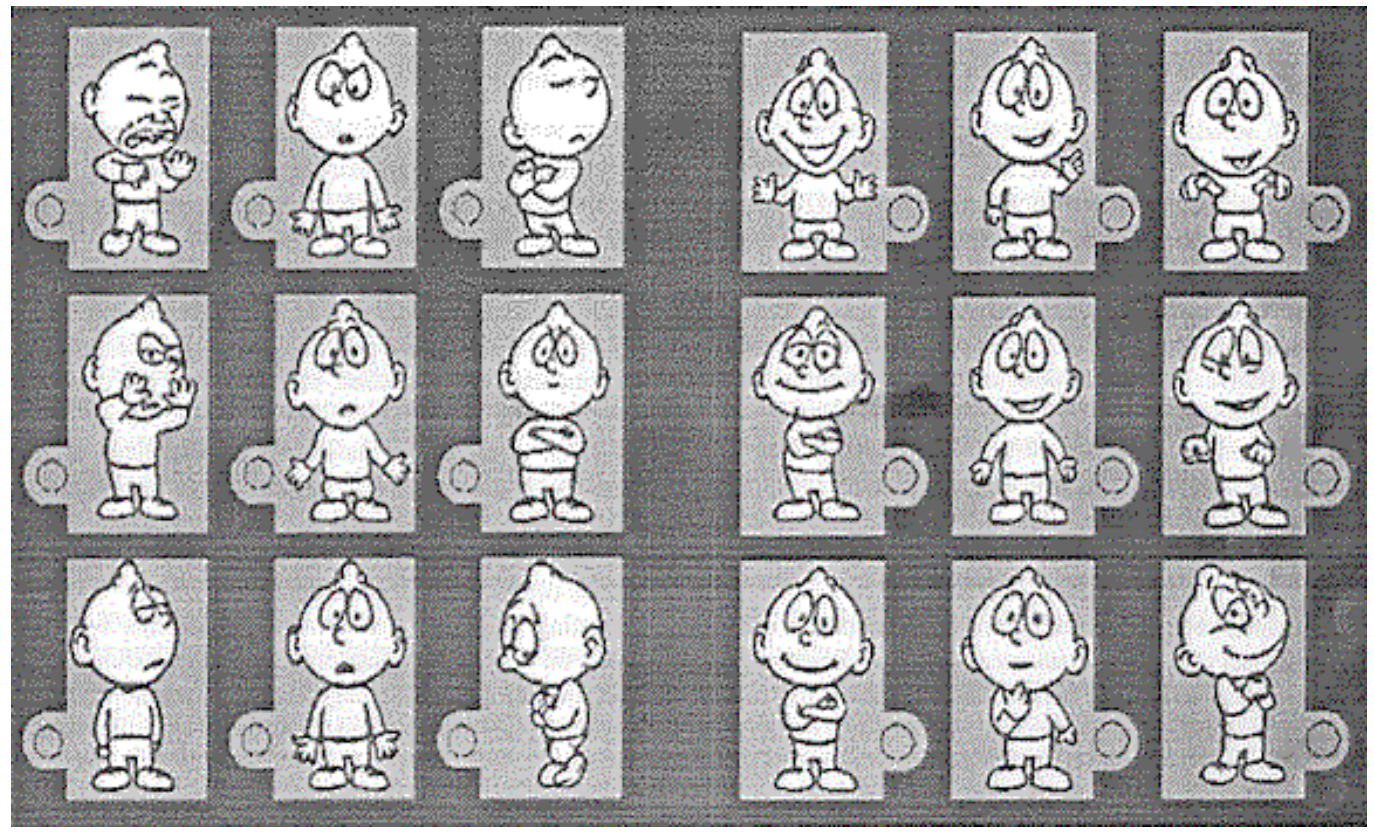

Fig. 2.6. PrEmo Emotion Animations. The 18 emotion animations used on the computer-based emotion method, PrEmo (Desmet et al., 2000).

The expression of these cartoon characters is based upon the research of Ekman and Friesen (1986) on universal expressions of emotion. The emotions discussed for universal expressions of emotion are based upon basic emotions (i.e. fear and happiness) while the emotions used in PrEmo are more subtle, in which case Desmet et al., (2000) decided to use facial and bodily expressions of emotions to magnify and clarify the targeted emotions. Additionally, the authors suggest measuring these specific emotions provides clues as to why certain products elicit certain emotions, eventually allowing designers, marketers, product developers, and sensory scientists the opportunity to compare the products and emotions to understand what attributes may have elicited these certain emotions in some products and not in others (Desmet et al., 2000). 
The PrEmo method accounts for issues with emotion testing which other methods have neglected, specifically using images to decrease wording ambiguity, facilitate use by different cultures, and limit cognitive involvement all through the use of animated images. Although this method has many benefits, it does only consist of 18 emotions which may not be enough to capture the entire consumer emotional experience with the product (King \& Meiselman, 2010); however, an appropriate or acceptable number of emotions is still debated. Additionally, PrEmo was developed for the consumer's emotion response to the appearance of the product and not necessarily for those emotions associated with purchasing or using the product. The PrEmo method is promising in its non-verbal approach to measuring emotions; however, further information is needed about additional emotion images and the effectiveness of this method when evaluating the emotions associated with purchasing and using a product.

\subsubsection{Eliciting Emotions with Odors and Pictures}

The sense of smell has long been associated with eliciting emotions (Croy et al., 2011) and as explored earlier olfaction and emotion processes occur in similar regions of the brain, including the OFC (Rolls \& Grabenhorst, 2008), thus Croy et al., (2011) was interested in the elicitation of emotions by odors and pictures. Participants were asked to list odors which they thought would elicit specified emotions: happiness, anger, disgust, sadness, anxiety, and surprise. A control group was asked to provide images which they thought would elicit the same emotions; the researchers hypothesized that it would be easier for participants to find visual cues which evoked different emotions compared to odors (Croy et al., 2011). Common groups for evoking happiness in the odor group were: plants (58\%) and food (26\%), and for disgust: death and waste (55\%) and culture 
products (15\%). Typical pictures used for happiness were: nature (25\%), humans (24\%), and plants (21\%), and for disgust: death and waste (48\%) and animals (34\%). Ultimately, participants were better able to name visual evokers rather than olfactory evokers for anger and sadness, suggesting the elicitation of different emotions is very complex and difficult to perform through the olfactory system (Croy et al., 2011).

Although this study does not provide ground-breaking information in the world of emotion, it does aid in the development of an emotion testing method through finding: 1) subjects are better able to connect emotions to pictures/visual cues compared to odor cues, and 2) the importance of not only a stimulus, but also the significance of the stimulus chosen for understanding emotional responses (Croy et al., 2011). Only basic emotions were used in this study, perhaps validation with additional/less basic emotions would provide further insight into the effectiveness of emotional stimuli. Lastly, perhaps reversing the method for connecting stimuli and emotion by asking subjects to list emotions evoked from certain aromas may help product development, sensory scientists, designers, and marketers understand what emotional response is expected from a certain stimuli.

Through reviewing these fundamentally different emotion testing methodologies, the following aspects appear to be paramount: 1) selection of just enough (amount not specified) emotions to capture the consumer experience to a specific product, 2) use of both positive and negative emotions to understand how product users and non-users will related to a product, and 3) the use of images to decrease ambiguity, confusion, and misinterpretation of emotion words and better evoke basic emotions compared to odors (Table 2.4). The clear connection seen between pictures/visual cues and emotions is 
consistent with the understanding of decision-making, reward mechanisms, visual, and emotion processing observed in the brain.

Table 2.4. Comparison of Emotion Methods. Comparison of critical aspects for each emotion method, it appears PrEmo method contains more of the important aspects of testing compared to the other mentioned methods.

\begin{tabular}{|l|c|c|c|c|c|}
\hline \multicolumn{7}{|c|}{ Comparison of Emotion Methods } \\
\hline & $\begin{array}{c}\text { Tested } \\
\text { with Food }\end{array}$ & $\begin{array}{c}\text { Used } \\
\text { Images }\end{array}$ & $\begin{array}{c}\text { Cultural } \\
\text { Ease }\end{array}$ & $\begin{array}{c}\text { Beyond Basic } \\
\text { Emotions }\end{array}$ & $\begin{array}{c}\text { Balanced } \\
+ \text { and - } \\
\text { emotions }\end{array}$ \\
\hline EsSense & & & & & \\
\hline $\begin{array}{l}\text { Best-Worst } \\
\text { Scaling }\end{array}$ & & & & & \\
\hline PrEmo & & & & & \\
\hline $\begin{array}{l}\text { Odors and } \\
\text { Pictures }\end{array}$ & & & & & \\
\hline
\end{tabular}

\subsection{Justification for Work}

There is an obvious need for Americans to reduce their sodium intake by a large margin. Although a total dietary modification is likely needed to achieve the recommended sodium intake levels, reducing sodium in a variety of popular foods could help Americans achieve their sodium intake goals. Mozzarella cheese is the most highly consumed cheese in the United States and is a good source of calcium and protein; however, the $8 \%$ DV sodium content in just one stick of mozzarella string cheese makes it a prime candidate for sodium reduction. Consumer acceptability for low or reduced sodium cheese products is typically quite low, and thus low sodium cheese products are not as successful as their full sodium counterpart. Perhaps the current questions sensory scientists are asking about low sodium cheese including acceptability, hedonics, and 
descriptive analysis are not clearly assessing why consumers do not like and/or will not repurchase low sodium cheese products. Considering the relationship between decisionmaking, reward mechanisms, and emotions in the brain, emphasis should be placed on capitalizing on this relationship to develop a method which dives deeper into the consumer's conceptualizations and emotions toward products.

In this thesis, a series of traditional sensory tests were performed to select a sensory transparent antimicrobial and a consumer acceptable flavor enhancer to develop a low sodium LMPS mozzarella cheese product. Additionally, a novel emotion testing method was developed which compared the use of images versus language for eliciting emotional responses in consumers. For validation of this method, imagery and language for emotion testing were compared across three product categories with commercially available products. Finally, the newly developed emotion testing method was implemented with the low sodium mozzarella cheese prototypes. By measuring consumer emotions as they related to nutritional alternative food products, such as low sodium mozzarella cheese, provided additional information beyond liking and acceptability and thus provided a greater understanding of the consumer's mindset. Low sodium cheese products are useless to the consumer if they are not enjoyable to eat; the goal of this novel emotion testing method is to determine if similar emotional responses typically experienced with the full sodium product can be manifested in the consumer when consuming the low sodium cheese product. 


\title{
Sensory Evaluation of Antimicrobials and Salt Replacers in Low Sodium Mozzarella Cheese
}

\begin{abstract}
Dietary sodium intake for most Americans is far beyond the recommended allowance, and many Americans are looking for foods which provide the same taste of traditional full sodium products but with lower sodium content. Considering the sodium contribution dairy foods make to the America diet and the amount of sodium in cheese, this study focused on the development and consumer sensory evaluation of low sodium LMPS mozzarella cheese. Four antimicrobials were compared through a series of affective and hedonic consumer tests to determine the most sensory transparent product with LMPS mozzarella cheese. Upon selection of the most appropriate antimicrobial, $\mathrm{NaCl}$ reduced and substituted mozzarella cheese systems were made using both direct acidification and bacteria cultured acidification cheeses. Subjects were asked hedonic and preference ranking questions regarding both styles of the low sodium mozzarella cheese, and based upon hedonic scores a recommendation for manufacturers was made.
\end{abstract}

Highlights: > Discrimination and affective testing of four antimicrobials on LMPS mozzarella cheese. > Hedonic testing with 6 variables for determining the most liked salt replacer. $>$ Salt replacer hedonic testing performed in direct acidification and bacteria culture acidification mozzarella cheese.

Keywords: sodium reduction, consumer sensory testing, antimicrobials, salt replacers, mozzarella cheese 


\subsection{Introduction}

Increased dietary sodium consumption is associated with hypertension which is a leading risk factor for stroke and cardiovascular disease, the two leading causes of death in the United States (CDC, 2012; USDA, 2005). Ninety percent of Americans are estimated to consume higher levels of sodium than recommended, with an approximate consumption rate of 3,300 $\mathrm{mg}$ of sodium daily, 1,000 $\mathrm{mg}$ more than the U.S. dietary guideline recommendations (CDC, 2012). High sodium intake is often contributed to the abundance of $\mathrm{NaCl}$ in a variety of typically consumed processed and convenience foods including: breads, processed meats, pizza, dairy products, soups, and snacks and restaurant foods (CDC, 2012). Dairy products alone are thought to contribute approximately $11 \%$ of the total sodium in American diets (Demott, 1985). Although mozzarella cheese does not have the highest sodium content compared to other cheeses, it does contain approximately $1.40 \% \mathrm{NaCl}$ (Cruz et al., 2011), resulting in roughly $8 \%$ of the Daily Value (DV) of sodium in one serving. Consequently, individuals looking to reduce dietary sodium often restrict dairy foods, specifically cheese due to the high sodium content (Katsiari et al., 1997). The dairy industry is in pursuit of methods to decrease the sodium content of cheeses which contain higher levels of sodium compared to other types of dairy products (Reddy\& Marth, 1993), and the industry has been relatively successful in reducing sodium in some cheeses including cheddar (Grummer et al., 2012) and feta (Katsiari et al., 1997).

Sensory consequences of sodium reduction in mozzarella cheese have not been well studied; however, the investigation of chemical composition and functional properties of LMPS mozzarella with partial substitution of $\mathrm{NaCl}$ with $\mathrm{KCl}$ has been 
evaluated (Ayyash \& Shah, 2011). Ayyash and Shah (2011) determined the use of $1 \mathrm{NaCl}: 1 \mathrm{KCl}$ and $1 \mathrm{NaCl}: 3 \mathrm{KCl}$ in mozzarella cheese had similar effects on all components tested including: chemical composition, organic acids profiles, functional properties, meltability and browning, but sensory testing was not performed. Sensory evaluation of this traditionally bland cheese is necessary in order to understand the most appropriate and effective method for reducing the sodium content in mozzarella cheese while still being acceptable to consumers.

Considering two traditional methods exist for lowering sodium in foods, reducing $\mathrm{NaCl}$ and substitution of $\mathrm{NaCl}$ (Cruz et al., 2011), this study will investigate the effect of reducing sodium in mozzarella cheese alone, and also the partial substitution of $\mathrm{NaCl}$ with three flavor modifiers. The main objectives of this study are to: 1) conduct sensory tests on four antimicrobials with mozzarella cheese to determine the most sensory transparent product, 2) perform sensory testing on sodium reduced and substituted mozzarella products using two types of mozzarella cheese (direct acidification and bacteria cultured acidification) with consumers to determine the most liked and preferred product, and 3) provide an antimicrobial and salt replacer formulation recommendation for manufacturers for lowering sodium content in LMPS mozzarella cheese. It is hypothesized that a salt substitute rather than sodium reduction alone will be more preferred by consumers and liking/preference scores for the reduced sodium cheese will be lower than a traditional full sodium LMPS mozzarella cheese. 


\subsection{Methodology}

\subsubsection{Materials: Antimicrobials}

Four commercially available antimicrobials were tested: Protect-M (Purac America, lot number: 1010001940), NovaGard CB1 (Danisco, lot number: CB1-032110), SEA-i F75 (Bienca, lot number:101027), and PuraQ Verdad RV75 (Purac America, lot number: 0910002224). Full salt and low salt LMPS mozzarella cheese from Saputo (Saputo Cheese USA Inc., Tulare, CA) was used for the antimicrobial sensory testing.

\subsubsection{Methods: Antimicrobials}

A series of sensory evaluation tests were completed to determine detectability and likeability of the different antimicrobials, sensory tests included: triangle, duo-trio, and 9point hedonic tests with product category users (Fig. 3.1). 


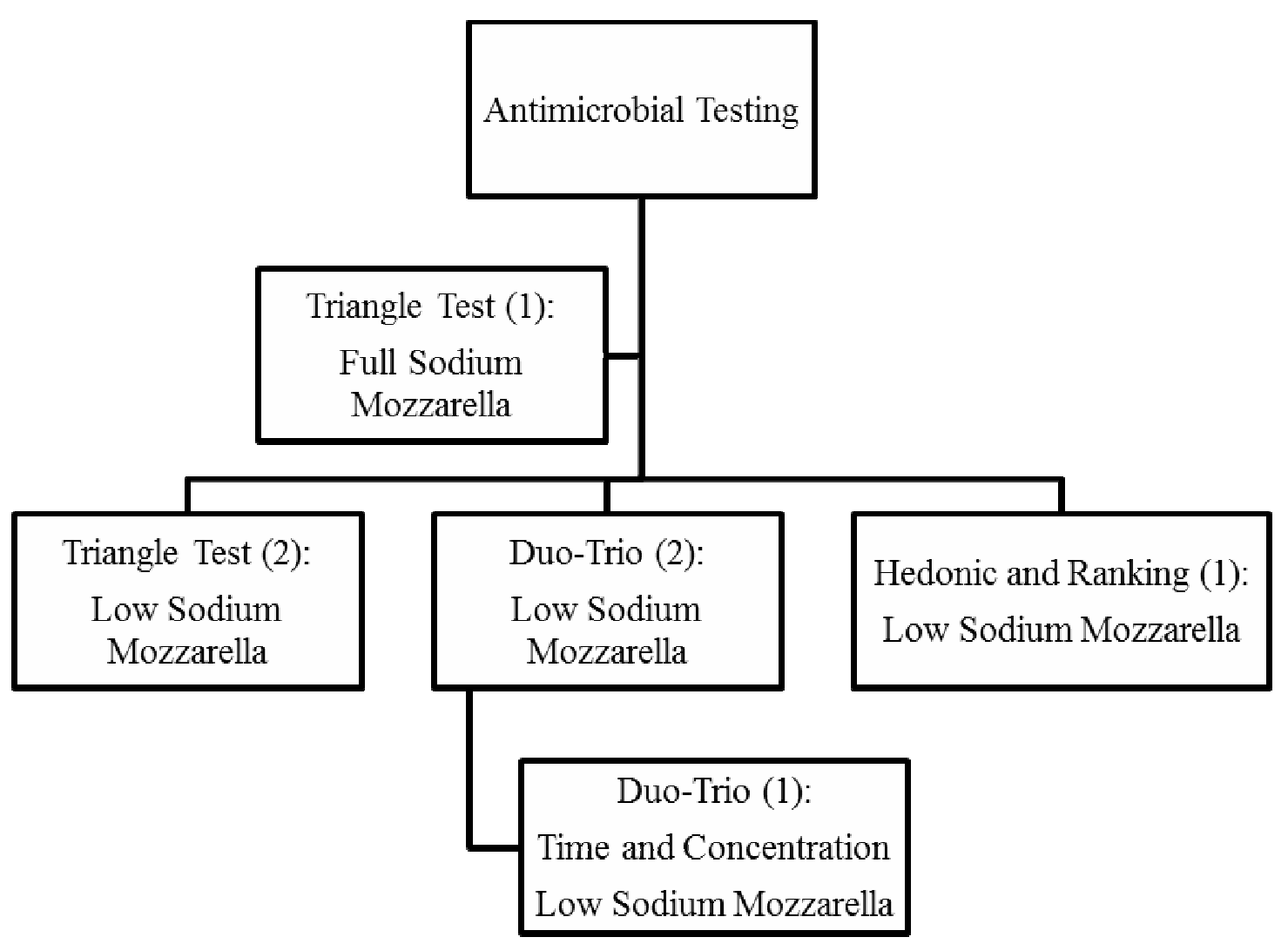

Fig. 3.1. Overview of Antimicrobial Sensory Testing. The number in the parentheses indicates the number of times the test was performed.

The antimicrobials and concentrations used were selected based upon challenge studies performed elsewhere and manufacturer suggested use recommendations: $1.0 \%$ NovaGard CB1, 0.2\% Protect-M, 0.25\% SEA-i F75, and 0.2\% PuraQ RV75.

Experimental antimicrobial use levels were $10 \%$ greater than manufacturer recommendation to potentially magnify any sensory differences when used with LMPS mozzarella cheese. The antimicrobial dip solutions were made with Sparkletts® (DS Waters of America, Inc., Atlanta, GA) distilled water and addition of the appropriate amount of the antimicrobial, the solution was stirred using a stir plate for two minutes or until homogenous. The homogenous antimicrobial solution was then poured into a large plastic container for dipping. The mozzarella cheese was cut into $3 / 4$ inch cubes then dipped in the antimicrobial solution for one minute with gentle agitation and another 
minute of soaking. The cheese cubes were strained from the solution and allowed to airdry at ambient temperature on labeled aluminum foil for 30-45 minutes or until no longer wet. Once the dipped cubes were dry, they were placed in two ounce clear plastic SOLO® cups (SOLO ${ }^{\circledR}$ Cup Company, Lake Forest, IL) with randomized 3-digit codes and cupped. Cheese samples were kept at $37^{\circ} \mathrm{F}$ overnight prior to sensory testing and were kept refrigerated until 30 minutes prior to testing. The control cheese was dipped in distilled water using the above procedure, the distilled water dipped samples will be referred to as "non-dipped" samples despite having been dipped in water; all testing preparations were completed the day prior to the sensory testing.

\subsubsection{Triangle Test with Full Sodium Mozzarella Cheese}

Two separate triangle testing sessions were performed using full sodium $(\sim 1.8 \%)$ LMPS mozzarella from Saputo, where each antimicrobial was tested with product category users defined as consuming mozzarella cheese "once every few months" to “everyday.” NovaGard CB1 and Protect-M were tested among consumers $(n=38)$ to determine if there was a detectable difference between cheeses containing an antimicrobial. Two triangle tests were performed where: 1) the NovaGard CB1 cheeses were compared to non-dipped cheese, and 2) the Protect-M cheeses were tested with nondipped cheese. A ten second delay was programmed into the test protocol between each sample set to decrease any carryover between samples. The SEA-i F75 and PuraQ RV75 antimicrobial triangle tests were performed two days later with a similar population $(n=30)$. Each session took approximately five minutes to complete. 


\subsubsection{Triangle Test with Low Sodium Mozzarella Cheese}

Two sensory testing sessions were performed with all four antimicrobials tested in each session on low sodium mozzarella cheese provided by Saputo. The mozzarella cheese product was made for commercial distribution, but had bypassed the brining stage of manufacturing and thus had lower sodium content (0.86\%) than typical Saputo LMPS mozzarella string cheese (1.6-1.8\%). In each session, subjects were randomly assigned the order of the triangle tests and all four antimicrobial triangle tests were completed at one session; each triangle test compared antimicrobial containing cheese to control cheese with a ten second delay between sample sets. The second session $(n=17)$ occurred two days after the first session $(n=24)$ and was performed as a pseudo-replicate of the first session to validate results, considering the sample population size was rather small. Each testing session took less than ten minutes to complete and subjects were product category users defined as consuming mozzarella string cheese "once every few months" to "every day."

\subsubsection{Duo-Trio Tests with Low Sodium Mozzarella Cheese}

Two sessions of duo-trio tests were performed with all four antimicrobials tested at each session, with each discrimination test comparing the antimicrobial cheese to the control cheese. All duo-trio tests used as a balance reference design, with the reference presented on the left for all subjects, and subjects were asked to indicate the sample which was different from the reference sample. Sample set order was randomized for each subject, and a mandatory ten second delay was programmed between each sample set. Subjects for session one $(n=30)$ and two $(n=28)$ included those who consume 
mozzarella string cheese "once every few months" to "every day." Session one and two had the same protocol with similar populations, session two was executed seven days after session one. Testing took participants less than ten minutes on average to complete.

\subsubsection{Hedonic Testing with Low Sodium Mozzarella Cheese}

Following the testing in 3.2.2.3, hedonic testing was implemented to understand which, if any, of the antimicrobials was most preferred. The low sodium mozzarella cheese was used for testing the four antimicrobials and the control (dipped in distilled water and dried). Subjects $(n=22)$ included those consuming mozzarella string cheese " 2 3 times per week" to "once every few months" were asked the following liking questions on a 9-point scale for each of the five cheese samples: overall acceptance, aroma, flavor, and aftertaste. Samples were presented using the William's design, were samples were randomized within and across subjects, and samples were presented one at a time with a ten second delay between each sample. Testing took approximately ten minutes for subjects to complete.

\subsubsection{Effect of Time and Concentration of Antimicrobial Dip on Discrimination}

In order to understand the effects of the soaking time and the concentration of the antimicrobial dip on the flavor of the mozzarella cheese, a study was performed using only the selected antimicrobial (SEA-i F75) at varying times and concentrations in the antimicrobial bath. The typical procedure, as stated previously, included placing unbrined cheese cubes in a $0.275 \%$ SEA-i F75 solution for 120 seconds prior to removal and air-

drying. For this experiment, a $2^{2}$ design was created with two factors of time: 30 seconds 
and 3 minutes of dipping, and two concentration factors: $50 \%$ less than recommended usage $(0.1375 \%)$, and $50 \%$ more than recommended usage $(0.4125 \%)$.

Table 3.1. Antimicrobial Time and Concentration Parameters. A two factorial design for understanding the effect of time and concentration of the antimicrobial dip, $50 \%$ is relative to the amount suggested by the manufacture and microbiology team $(0.275 \%)$.

\begin{tabular}{|c|c|c|c|}
\hline \multicolumn{4}{|c|}{ Antimicrobial Time and Concentration Parameters } \\
\hline & & Con & ntration \\
\hline \multirow{3}{*}{ 兽 } & & $50 \%$ less & $50 \%$ more \\
\hline & \multirow{2}{*}{\begin{tabular}{|l}
30 seconds \\
3 minutes
\end{tabular}} & $50 \%$ less@30s & $50 \%$ more @ $30 \mathrm{~s}$ \\
\hline & & $50 \%$ less@3 min & $50 \%$ more @ $3 \mathrm{~min}$ \\
\hline
\end{tabular}

Cheeses were treated with same protocol as describe in 3.2.2. Two duo-trio test sessions were completed on the same day (morning and afternoon sessions) with product users $(n=31)$, defined as consuming mozzarella string cheese "everyday" to "every few months." The second session was used as a replicate of the first, with the same test design, products, and subjects, but different blinding codes. The duo-trio tests were presented as a balanced reference design with the reference sample on the left for all subjects. One session of testing took subjects approximately ten minutes to complete.

\subsubsection{Materials: Salt Replacers}

In the next round of sensory testing, three salt replacers were compared: $\mathrm{NaCl} / \mathrm{KCl}$ blend ( $\mathrm{KCl}$ by Morton Salt, lot number: APR102HK01), ALTA ${ }^{\mathrm{TM}} 2345$ (Kerry, ID number: s-341825), and Salona (BK Giulini, lot number: 15APR2011-F); a low sodium mozzarella cheese $(0.8 \%$ salt per curd weight $)$ using the reduced $\mathrm{NaCl}$ method and a full sodium mozzarella cheese (1.9\% salt per curd weight) were used as the controls in testing (Table 3.2). 
Table 3.2. Specifications for Salt Replacer Cheese Variables. Six variables were made for testing with consumers to determine which cheese would be liked most. Blends of $\mathrm{NaCl}$ and a salt replacer were calculated to fulfill the maximum limit of sodium allowed in a low sodium cheese; some salt replacers contained sources of sodium.

\begin{tabular}{|c|c|c|c|c|c|c|c|}
\hline \multicolumn{8}{|c|}{ Specifications for Salt Replacer Cheese Variables } \\
\hline $\begin{array}{l}\text { Sample } \\
\text { Number }\end{array}$ & $\begin{array}{c}\text { Type } \\
\text { of } \\
\text { Cheese }\end{array}$ & $\begin{array}{c}\text { Amount } \\
\text { of NaCl } \\
\text { (per } \\
201 \mathrm{bs} \\
\text { cheese } \\
\text { block) }\end{array}$ & $\begin{array}{c}\text { Salt } \\
\text { Replacer }\end{array}$ & $\begin{array}{c}\text { Amount } \\
\text { of Salt } \\
\text { Replacer } \\
\text { (per 20lbs } \\
\text { cheese } \\
\text { block) }\end{array}$ & $\begin{array}{c}\text { Calculated } \\
\% \mathrm{NaCl} \\
\text { Content }\end{array}$ & $\begin{array}{l}\text { Calc Na } \\
\text { Content } \\
(\mathrm{NaCl} \sim \\
40 \% \mathrm{Na})\end{array}$ & $\begin{array}{l}\text { Dipped } \\
\text { in } \\
\text { SEA-i } \\
\text { F75 }\end{array}$ \\
\hline 1 & $\begin{array}{c}\text { Full } \\
\text { Sodium }\end{array}$ & $250 \mathrm{~g}$ & None & N/A & $2.8 \%$ & $1.1 \%$ & No \\
\hline 2 & $\begin{array}{c}\text { Low } \\
\text { Sodium }\end{array}$ & $117.5 \mathrm{~g}$ & None & N/A & $1.3 \%$ & $0.53 \%$ & No \\
\hline 3 & $\begin{array}{c}\text { Low } \\
\text { Sodium }\end{array}$ & $117.5 \mathrm{~g}$ & None & N/A & $1.3 \%$ & $0.53 \%$ & Yes \\
\hline 4 & $\begin{array}{c}\text { Low } \\
\text { Sodium }\end{array}$ & $100 \mathrm{~g}$ & $\mathrm{KCl}$ & $81 \mathrm{~g}$ & $1.1 \%$ & $0.44 \%$ & Yes \\
\hline 5 & $\begin{array}{c}\text { Low } \\
\text { Sodium }\end{array}$ & $75 \mathrm{~g}$ & $\begin{array}{c}\text { ALTA }^{\mathrm{TM}} \\
2345\end{array}$ & $20.4 \mathrm{~g}$ & $0.83 \%$ & $0.33 \%$ & Yes \\
\hline 6 & $\begin{array}{c}\text { Low } \\
\text { Sodium }\end{array}$ & $90 \mathrm{~g}$ & Salona & $77.6 \mathrm{~g}$ & $0.99 \%$ & $0.40 \%$ & Yes \\
\hline
\end{tabular}

From the series of sensory tests performed for selecting a sensory transparent antimicrobial, the antimicrobial selected (SEA-i F75) was used in conjunction with the \#3 low sodium cheese, \#4 NaCl/KCl blend ( 1:1) cheese, \#5 ALTA ${ }^{\mathrm{TM}}$ cheese, and \#6

Salona cheese to understand any confounding effect which may occur when both the antimicrobial and salt replacer were used within the cheese matrix. These salt replacers were incorporated into the LMPS mozzarella cheese using a cooker-stretcher (Blentech, model CC-45) made in the California Polytechnic State University - San Luis Obispo DPTC pilot plant; a total of six variables were made and tested for liking with consumers.

\subsubsection{Methods: Salt Replacers}

Both direct acidified (5\% acetic acid) and bacteria cultured (Chr. Hansen, ST-M6 at $0.027 \%$ of milk weight) mozzarella cheese were made for this study to understand if 
the type of mozzarella cheese was a factor when asking consumers his/her liking and ranking scores for each of the salt replacer variables.

\subsubsection{Hedonic and Ranking in Direct Acidified and Bacteria Cultured Mozzarella}

\section{Cheese}

Hedonic and ranking tests were performed with 98 subjects, all of whom consumed cheese at least "once per month" and 91 whom consumed mozzarella string cheese "everyday" to "every few months." Subjects were first asked hedonic and ranking questions in regards to the six variables of salt replacers in the bacteria cultured cheese. After a mandatory one minute break, the subjects were asked hedonic and ranking questions in regards to the six salt replacer variables in the direct acidified cheese. Testing took subjects 20-25 minutes on average to complete.

\subsubsection{General Sensory Testing Procedures}

All sensory tests were approved by the California Polytechnic State University San Luis Obispo Institutional Review Board prior to testing; additionally, all sensory tests were implemented in white sensory booths with unique lighting and airflow to aid in sensory testing and participants were provided unsalted crackers, drinking water, and an expectoration cup to decrease sensory fatigue and carryover between samples. Testing was performed on Compusense® 5.2 Plus software (Compusense Inc., Guelph, Ontario, Canada) and subjects received candy or food for participating in the studies. 


\subsection{Data Analysis}

Data analysis for all triangle tests, duo-trio tests, hedonic and ranking scores were analyzed using the Compusense ${ }^{\circledR} 5.2$ software. Compusense ${ }^{\circledR}$ analyses of the triangle and duo-trio tests were performed based upon binomial distribution tables in the sensory literature (i.e. Roessler et al., 1978), the hedonic scores were analyzed using Analysis of Variance (ANOVA) with Tukey's HSD at 5\% significance for all post hoc comparisons, and ranking analysis was calculated by the Friedman's Test using a chi-square distribution.

\subsection{Results}

\subsubsection{Antimicrobial Sensory Results}

The series of antimicrobial sensory testing yielded the results for selecting a sensory transparent antimicrobial.

\subsubsection{Triangle Test with Full Sodium Mozzarella Cheese}

Consumers were not able to detect a difference $(\alpha=0.05)$ between the NovaGard CB1 cheese, and non-dipped cheese, Protect-M and non-dipped cheese, SEA-i F75 and non-dipped cheese, or PuraQ RV75 and non-dipped cheese (Table 3.3). Although consumers were not able to detect a difference between any of the dipped and non-dipped cheeses, it appears consumers were correct more often in discriminating between the Protect-M dipped samples and non-dipped samples compared to the NovaGard CB1 triangle test. Based upon these tests alone, if one antimicrobial had to be selected for being sensory transparent the NovaGard CB1 would be chosen due to the highest number 
of incorrect answers for that day, suggesting consumers were guessing and could not actually detect a difference between the samples. The triangle tests for comparing SEA-i F75 to non-dipped cheese and PuraQ RV75 to non-dipped cheese did not result in identifying an antimicrobial which was significantly different $(\alpha=0.05)$ from the nondipped cheese. Based upon these triangle tests alone, SEA-i F75 appears to be more difficult for consumers to detect a difference between the dipped and non-dipped samples because of the higher number of incorrect answers compared to the number of incorrect answers form the PuraQ RV75 triangle test (Table 3.3).

Table 3.3. Day 1 and Day 2 Sensory Testing with Antimicrobials. Consumers were not able to determine a difference between the samples in the triangle test, for 38 consumers 19 correct answers would have suggested a significant detectable difference between the samples; for 30 consumers 15 correct answers were needed to reach significance.

\begin{tabular}{|c|c|c|c|c|}
\hline & \multicolumn{2}{|c|}{ Day 1 and Day 2 Sensory Testing with Antimicrobials } \\
\hline Triangle Test & $\begin{array}{c}\text { NovaGard CB1 } \\
\text { w/o NovaGard CB1 }\end{array}$ & $\begin{array}{c}\text { Protect-M } \\
\text { w/o Protect-M }\end{array}$ & $\begin{array}{c}\text { SEA-i F75 } \\
\text { w/o SEA-i F75 }\end{array}$ & $\begin{array}{c}\text { PuraQ R V75 } \\
\text { w/o PuraQ R 75 }\end{array}$ \\
\hline Incorrect & 31 & 23 & 18 & 16 \\
\hline Correct & 7 & 15 & 12 & 14 \\
\hline Total & 38 & 38 & 30 & 30 \\
\hline p-value & 0.9870 & 0.2610 & 0.2760 & 0.0900 \\
\hline
\end{tabular}

\subsubsection{Triangle Test with Low Sodium Mozzarella Cheese}

Two sensory testing sessions were carried out on low sodium mozzarella cheese where all four antimicrobials were compared in each session. The first triangle test session did not yield any significant differences $(\alpha=0.05)$ between the antimicrobial cheese and the non-dipped cheese (Table 3.4). Additionally, two antimicrobials were equally as difficult to discriminate from the non-dipped cheese: Protect-M and SEA-i F75, with 17 incorrect answers each. 
Table 3.4. Session 1: Triangle Test with All Antimicrobials. Consumers were not able to detect a difference between any of the antimicrobial dipped cheeses and non-dipped cheeses. With testing 24 consumers, 13 correct answers were necessary for determining a significant difference between the samples.

\begin{tabular}{|c|c|c|c|c|}
\hline \multicolumn{5}{|c|}{ Session 1: Triangle Test with All Antimicrobials } \\
\hline Triangle Test & $\begin{array}{c}\text { NovaGard CB1 } \\
\text { w/o NovaGard CB1 }\end{array}$ & $\begin{array}{c}\text { Protect-M } \\
\text { w/o Protect-M }\end{array}$ & $\begin{array}{c}\text { SEA-i F75 } \\
\text { w/o SEA-i F75 }\end{array}$ & $\begin{array}{c}\text { PuraQ RV75 } \\
\text { w/o PuraQ RV75 }\end{array}$ \\
\hline Incorrect & 13 & 17 & 17 & 14 \\
\hline Correct & 11 & 7 & 7 & 10 \\
\hline Total & 24 & 24 & 24 & 24 \\
\hline p-value & 0.1400 & 0.7370 & 0.7370 & 0.2540 \\
\hline
\end{tabular}

Based upon these triangle tests alone, Protect-M and SEA-i F75 were the most difficult for consumers to detect from non-dipped cheese, and thus could be suggested as the most sensory transparent antimicrobials. This test was pseudo-replicated with 33 consumers and subjects again were not able to detect a difference between any of the antimicrobial cheeses and non-dipped cheeses (Table 3.5).

Table 3.5. Session 2: Triangle Test with All Antimicrobials. Consumers were not able to detect a difference between the antimicrobial dipped cheeses and the non-dipped cheeses. With 33 consumers, 17 correct answers were needed to determine a significant difference between the samples.

\begin{tabular}{|c|c|c|c|c|}
\hline \multicolumn{5}{|c|}{ Session 2: Triangle Test with All Antimicrobials } \\
\hline Triangle Test & $\begin{array}{c}\text { NovaGard CB1 } \\
\text { w/o NovaGard CB1 }\end{array}$ & $\begin{array}{c}\text { Protect-M } \\
\text { w/o Protect-M }\end{array}$ & $\begin{array}{c}\text { SEA-i F75 } \\
\text { w/o SEA-i F75 }\end{array}$ & $\begin{array}{c}\text { PuraQ RV75 } \\
\text { w/o PuraQ RV75 }\end{array}$ \\
\hline Incorrect & 17 & 24 & 23 & 20 \\
\hline Correct & 16 & 9 & 10 & 13 \\
\hline Total & 33 & 33 & 33 & 33 \\
\hline p-value & 0.0506 & 0.8201 & 0.7034 & 0.2838 \\
\hline
\end{tabular}

Although no significant differences were detected, a slightly clearer distinction between transparencies of the antimicrobials was observed. NovaGard CB1 with 16 correct answers had the closest detectable difference between the samples, while the other antimicrobials were more difficult to discriminate from the non-dipped cheeses; ProtectM was the most difficult to distinguish considering the 24 incorrect answers to the triangle test and SEA-i F75 close behind with 23 incorrect answers. Based upon the two 
triangle test sessions, Protect-M and SEA-i F75 appeared consistently difficult for consumers to detect from non-dipped cheese.

\subsubsection{Duo-Trio with Low Sodium Mozzarella Cheese}

Two duo-trio testing sessions were performed to validate the findings in 3.4.1.2, with 30 consumers comparing all four antimicrobial cheeses to non-dipped cheeses. In the first duo-trio testing session, overall the 30 consumers were unable to identify which sample was different from the reference sample (Table 3.6).

Table 3.6. Session 1: Duo-Trio Test with All Antimicrobials. Consumers were not able to determine with sample was different from the reference when comparing dipped and non-dipped cheeses. With 30 consumers, 20 correct responses were needed to determine a significant difference between the samples.

\begin{tabular}{|c|c|c|c|c|}
\hline \multicolumn{5}{|c|}{ Session 1: Duo-Trio Test with All Antimicrobials } \\
\hline Duo-Trio Test & $\begin{array}{c}\text { NovaGard CB1 } \\
\text { w/o NovaGard CB1 }\end{array}$ & $\begin{array}{c}\text { Protect-M } \\
\text { w/o Protect-M }\end{array}$ & $\begin{array}{c}\text { SEA-i F75 } \\
\text { w/o SEA-i F75 }\end{array}$ & $\begin{array}{c}\text { PuraQ RV75 } \\
\text { w/o PuraQ R V75 }\end{array}$ \\
\hline Same & 11 & 12 & 13 & 16 \\
\hline Different (Correct) & 19 & 18 & 17 & 14 \\
\hline Total & 30 & 30 & 30 & 30 \\
\hline p-value & 0.1000 & 0.1810 & 0.2920 & 0.7080 \\
\hline
\end{tabular}

Although consumers were not able to detect a difference between the dipped and nondipped samples, based upon this duo-trio test session only, PuraQ RV75 appeared to be the most difficult for consumers to distinguish from the non-dipped cheese sample, with only 14 correct answers. NovaGard CB1, Protect-M, and SEA-i F75 were not as difficult for consumers to detect a difference; NovaGard CB1 was one correct answer shy of being significant. Based upon this series of duo-trio tests, PuraQ RV75 appeared to be the most difficult for consumers to detect compared to non-dipped cheese.

A pseudo-replicate of this test was performed with 28 consumers, and again consumers were not able to detect a significant difference between any of the antimicrobial cheeses compared to non-dipped cheeses (Table 3.7). 
Table 3.7. Session 2: Duo-Trio Test with All Antimicrobials. Consumers were not able to detect a difference between any of the antimicrobial dipped cheeses and non-dipped cheeses. With 28 consumers, 19 correct answers were needed to detect a significant difference between the samples.

\begin{tabular}{|c|c|c|c|c|}
\hline \multicolumn{5}{|c|}{ Session 2: Duo-Trio Test with All Antimicrobials } \\
\hline Duo-Trio Test & $\begin{array}{c}\text { NovaGard CB1 } \\
\text { w/o NovaGard CB1 }\end{array}$ & $\begin{array}{c}\text { Protect-M } \\
\text { w/o Protect-M }\end{array}$ & $\begin{array}{c}\text { SEA-i F75 } \\
\text { w/o SEA-i F75 }\end{array}$ & $\begin{array}{c}\text { PuraQ RV75 } \\
\text { wo PuraQ RV75 }\end{array}$ \\
\hline Same & 15 & 12 & 16 & 13 \\
\hline Different (Correct) & 13 & 16 & 12 & 15 \\
\hline Total & 28 & 28 & 28 & 28 \\
\hline p-value & 0.7140 & 0.2860 & 0.8280 & 0.4250 \\
\hline
\end{tabular}

Considering this series of duo-trio tests only, NovaGard CB1 with 15 and SEA-i F75

with 16 incorrect answers appeared to be the most difficult for consumers to detect a difference between the dipped and non-dipped cheeses. Based upon the two duo-trio testing sessions, NovaGard CB1 and SEA-i F75 were consistently difficult for consumers to detect compared to the non-dipped cheese.

\subsubsection{Hedonic Testing with Low Sodium Mozzarella Cheese}

The hedonic test with 22 subjects provided little information beyond what the discrimination tests had provided, with the only significant difference in liking between the four antimicrobial samples and a control was in aroma liking $\left(\mathrm{F}_{4,84}=3.29\right.$, $\mathrm{P}=0.0149$ ); all other liking attributes did not differ between the samples at $\alpha=0.05$. Considering a significant difference in aroma liking was determined, a post hoc comparison was performed and the results suggested SEA-i F75 aroma was liked significantly more than the NovaGard CB1 aroma (Table 3.8). 
Table 3.8. Hedonic Test - "Aroma" Post Hoc Analysis. Post hoc comparison using Tukey's HSD at 5\% significance determined the SEA-i F75 aroma was liked significantly more than the NovaGard CB1 aroma. Samples which do not share a difference letter are significantly different.

\begin{tabular}{|c|c|c|l|}
\hline \multicolumn{4}{|c|}{ Hedonic Test - "Aroma" Post Hoc Analysis } \\
\hline Sample & Mean & SD & Differences \\
\hline SEA-i F75 & 6.27 & 1.695 & A \\
\hline PuraQ RV75 & 6.23 & 1.541 & A B \\
\hline Protect M & 5.95 & 1.676 & A B \\
\hline Control & 5.64 & 1.465 & A B \\
\hline NovaGard CB1 & 5.45 & 1.654 & B \\
\hline
\end{tabular}

Although this hedonic test did not provide major contributions about each of the samples, it was helpful for distinguishing liking between two samples which were similar in the discrimination test: SEA-i F75 and NovaGard CB1. These samples had similar discrimination results, but the hedonic information helps in the selection of a sensory transparent and better liked product; based upon this information SEA-i F75 appears to be better liked than NovaGard CB1 and was consistently difficult for consumers to detect in discrimination testing.

\subsubsection{Effect of Time and Concentration of Antimicrobial Dip on Discrimination}

Little information was available on the effect of time and concentration of the SEA-i F75 on the discrimination ability between dipped and non-dipped cheeses. The $2^{2}$ design test determined that consumers were not able to detect a difference between the $50 \%$ less concentration with 30 seconds, 50\% more concentration with 30 seconds, and $50 \%$ less concentration with 3 minutes compared to non-dipped cheeses; but a significant difference $(\alpha=0.05)$ was determined between the $50 \%$ more concentration with 3 minute dip cheese compared to non-dipped cheese (Table 3.9). 
Table 3.9. Time and Concentration Duo-Trio Results. Subjects were not able to detect a difference between the first three variables, but a significant difference between the cheeses dipped in $50 \%$ more concentration for 3 minutes and the non-dipped cheese was detected. With 31 subjects, 21 correct answers were needed to determine a significant difference, and the last variable did meet this criteria.

\begin{tabular}{|c|c|c|c|c|}
\hline \multicolumn{5}{|c|}{ Time and Concentration Duo-Trio Results } \\
\hline Duo-Trio Test & $\begin{array}{c}\mathbf{5 0 \%} \text { less [] } \\
\mathbf{3 0} \mathbf{~ s e c}\end{array}$ & $\begin{array}{c}\mathbf{5 0 \%} \text { more [ ] } \\
\mathbf{3 0} \mathbf{~ s e c}\end{array}$ & $\begin{array}{c}\mathbf{5 0 \%} \text { less [ ] } \\
\mathbf{3} \text { min }\end{array}$ & $\begin{array}{c}\mathbf{5 0 \%} \text { more [ ] } \\
\mathbf{3} \mathbf{~ m i n}\end{array}$ \\
\hline Same & 18 & 16 & 14 & 10 \\
\hline Different (Correct) & 13 & 15 & 17 & 21 \\
\hline Total & 31 & 31 & 31 & 31 \\
\hline p-value & 0.8590 & 0.6400 & 0.3600 & $\mathbf{0 . 0 3 5 0}$ \\
\hline
\end{tabular}

The results of this study provided better insight into the time and concentration effects of using SEA-i F75, suggesting careful observation of the amount of time and the concentration at which the cheese is in the SEA-i F75 bath. Increasing either the concentration by $50 \%$ or the time from 2 minutes to three minutes appears to not have an effect on the discrimination differences to non-dipped cheese; however, when both concentration and time are increased, consumers were able to detect a difference. This information effects not only sensory, but also the procedures for the microbiology and manufacturing teams.

\subsubsection{Salt Replacer Sensory Results}

Consumer acceptability of the cheeses was performed with both direct acidified and bacteria cultured mozzarella cheese and the same variables were used in both tests (refer to Table 3.2).

\subsubsection{Hedonic and Ranking Scores for Direct Acidified Mozzarella Cheese}

When comparing the six variables in the direct acidified cheese an overall significant difference was found for overall acceptability $\left(\mathrm{F}_{5,480}=31.44, \mathrm{P}<0.0001\right)$, 
overall flavor $\left(\mathrm{F}_{5,480}=28.74, \mathrm{P}<0.0001\right)$, and saltiness liking $\left(\mathrm{F}_{5,480}=31.74, \mathrm{P}<0.0001\right)$, Tukey's HSD at 5\% significance was used for post hoc analysis (Table 3.10).

For overall acceptability, the full sodium mozzarella direct acidified cheese was liked significantly more than the other cheeses, while the $\mathrm{NaCl} / \mathrm{KCl}$ blend, Salona, and ALTA $^{\mathrm{TM}}$ were not significantly different from one another. The ALTA ${ }^{\mathrm{TM}}$ and low sodium (non-dipped) cheeses were not significantly different from one another, and low sodium non-dipped cheese was not significantly different from low sodium dipped mozzarella cheese.

The overall flavor liking scores yielded similar results to the overall acceptability scores, where the full sodium cheese was liked significantly more than the other variables, and the low sodium cheeses (dipped and non-dipped) were liked the least. The $\mathrm{NaCl} / \mathrm{KCl}$ blend, Salona, ALTA ${ }^{\mathrm{TM}}$, and low sodium (non-dipped) cheeses were not significantly different from each other for overall flavor liking. Lastly, the low sodium (non-dipped) was not significantly different from the low sodium dipped cheese.

The saltiness liking scores for the direct acidified cheeses were similar to those of the overall liking, and overall flavor scores with the full sodium cheese having the most liked saltiness flavor, and the low sodium cheeses having the least liked saltiness flavor. The $\mathrm{NaCl} / \mathrm{KCl}$ blend, Salona, and $\mathrm{ALTA}^{\mathrm{TM}}$ were not significantly different from one another, while ALTA ${ }^{\mathrm{TM}}$ and the low sodium (non-dipped) cheese were not significantly different, and the low sodium (non-dipped) cheese was not significantly different from the low sodium (dipped) samples. 
Table 3.10. Direct Acid Hedonic Test Post Hoc Analysis. Tukey's HSD at 5\% was used for the post hoc analysis; samples with different letter differences are significantly different $(\alpha=0.05)$ from one another. An * indicates the sample was dipped in $0.275 \%$ SEA-i solution prior to testing.

\begin{tabular}{|c|c|c|c|c|c|}
\hline \multicolumn{6}{|c|}{ Direct Acid Hedonic Test Post Hoc Analysis } \\
\hline \multicolumn{2}{|c|}{ Overall Acceptability } & \multicolumn{2}{c|}{ Overall Flavor } & \multicolumn{2}{c|}{ Saltiness } \\
\hline Sample & Mean \pm SD & Sample & Mean \pm SD & Sample & Mean \pm SD \\
\hline Full Sodium & $5.67 \pm 1.772 \mathrm{a}$ & Full Sodium & $5.67 \pm 1.852 \mathrm{a}$ & Full Sodium & $5.82 \pm 1.848 \mathrm{a}$ \\
\hline $\mathrm{NaC} / \mathrm{KCl}$ blend & $4.69 \pm 1.922 \mathrm{~b}$ & $\mathrm{NaCl} / \mathrm{KCl}$ blend & $4.42 \pm 1.836 \mathrm{~b}$ & $\mathrm{NaC} / \mathrm{KCl}$ blend & $4.73 \pm 1.729 \mathrm{~b}$ \\
\hline Salona* & $4.56 \pm 1.695 \mathrm{~b}$ & Salona* & $4.41 \pm 1.749 \mathrm{~b}$ & Salona* & $4.60 \pm 1.662 \mathrm{~b}$ \\
\hline Alta* $^{*}$ & $4.19 \pm 1.722 \mathrm{bc}$ & Alta $^{*}$ & $4.15 \pm 1.782 \mathrm{~b}$ & Alta* & $4.24 \pm 1.632 \mathrm{bc}$ \\
\hline Low Sodium & $3.91 \pm 1.72 \mathrm{~cd}$ & Low Sodium & $3.87 \pm 1.76 \mathrm{bc}$ & Low Sodium & $3.93 \pm 1.757 \mathrm{~cd}$ \\
\hline Low Sodium* & $3.49 \pm 1.634 \mathrm{~d}$ & Low Sodium* & $3.52 \pm 1.69 \mathrm{c}$ & Low Sodium* & $3.63 \pm 1.833 \mathrm{~d}$ \\
\hline
\end{tabular}

Based upon the hedonic scores, the full sodium cheese was consistently the most

liked, while the two low sodium cheeses (dipped and non-dipped) were the least liked and the three salt replacers $\left(\mathrm{NaCl} / \mathrm{KCl}, \mathrm{ALTA}^{\mathrm{TM}}\right.$, and Salona) were consistently not different from each other.

The Friedman's rank test indicated a significant difference in ranking scores (Chi$\left.\mathrm{Sq}_{5}=88.26, \mathrm{P}<0.0001\right)$ when comparing all variables, and Tukey's HSD at 5\% significance was used for the post hoc analysis (Table 3.11).

Table 3.11. Direct Acid Ranking Scores Post Hoc Analysis. Tukey's HSD at 5\% was use for post hoc analysis; samples with different letter differences are significantly different (alpha $=0.05$ level) from one another. A higher mean score indicates a lower liking, while a lower mean indicates a higher liking. An * indicates the sample was dipped in $0.275 \%$ SEA-i solution prior to testing.

\begin{tabular}{|c|c|c|}
\hline \multicolumn{2}{|c|}{ Direct Acid Ranking Scores - Post Hoc Analysis } \\
\hline Sample & Mean & Differences \\
\hline Low Sodium* & 436.00 & A \\
\hline Low Sodium & 389.00 & A B \\
\hline Alta $^{*}$ & 361.00 & B C \\
\hline Salona $^{*}$ & 338.00 & B C \\
\hline NaCl/KCl blend* & 301.00 & \multicolumn{2}{|c|}{ C } \\
\hline Full Sodium & 212.00 & \multicolumn{2}{|c|}{ D } \\
\hline
\end{tabular}

The ranking scores indicate similar information from the hedonic scores, with the full sodium cheese having the lowest mean score and thus was more preferred than the 
other samples, and the low sodium cheeses (dipped and non-dipped) were preferred the least. The full sodium cheese was preferred significantly more than the other cheeses, while the $\mathrm{NaCl} / \mathrm{KCl}$ blend, Salona, and $\mathrm{ALTA}^{\mathrm{TM}}$ were not significantly different in ranking scores. The ALTA ${ }^{\mathrm{TM}}$ and low sodium (non-dipped) cheese were not significantly different and the low sodium (non-dipped) cheese was not significantly different from the low sodium (dipped) cheese.

\subsubsection{Hedonic and Ranking Scores for Bacteria Cultured Mozzarella Cheese}

An overall significant difference was found for overall acceptability $\left(\mathrm{F}_{5,485}=\right.$ 41.54, $\mathrm{P}<0.0001)$, overall flavor $\left(\mathrm{F}_{5,485}=48.81, \mathrm{P}<0.0001\right)$, and saltiness liking $\left(\mathrm{F}_{5,485}=\right.$ 45.72, $\mathrm{P}<0.0001)$ when comparing the six variables in the bacteria cultured cheese, Tukey's HSD at 5\% significance was used for post hoc analysis (Table 3.12).

The overall acceptability liking indicated the full sodium bacteria cultured cheese was liked significantly more than the other cheeses, while the low sodium (dipped and non-dipped) cheeses were liked the least. The Salona, ALTA ${ }^{\mathrm{TM}}$, and $\mathrm{NaCl} / \mathrm{KCl}$ blend were not significantly different from one another.

The overall flavor liking scores were similar to those found in the overall acceptability test, with the full sodium mozzarella cheese was liked significantly more than the other cheeses, and the two low sodium cheeses (dipped and non-dipped) were liked the least. Again, Salona, ALTA ${ }^{\mathrm{TM}}$, and $\mathrm{NaCl} / \mathrm{KCl}$ blend were not significantly different from one another.

The saltiness liking scores indicated similar information found from the overall acceptability and overall flavor liking scores, where the full sodium mozzarella cheese 
saltiness was liked significantly more than the other cheeses. The low sodium cheeses (dipped and non-dipped) had the lowest saltiness liking scores compared to the other variables. No significant difference in saltiness liking was observed between the Salona, $\mathrm{ALTA}^{\mathrm{TM}}$, and $\mathrm{NaCl} / \mathrm{KCl}$ blend.

Table 3.12. Bacteria Cultured Hedonic Test Post Hoc Analysis. Tukey's HSD at 5\% was used for the post hoc comparison; samples with different letter differences are significantly different (alpha $=0.05$ level). An * indicates samples which have been dipped in SEA-i $0.275 \%$ solution prior to testing.

\begin{tabular}{|c|c|c|c|c|c|}
\hline \multicolumn{6}{|c|}{ Bacteria Cultured Hedonic Test Post Hoc Analysis } \\
\hline Overall Acceptability & \multicolumn{2}{c|}{ Overall Flavor } & \multicolumn{2}{c|}{ Saltiness } \\
\hline Sample & Mean \pm SD & Sample & Mean \pm SD & Sample & Mean \pm SD \\
\hline Full Sodium & $5.59 \pm 1.989 \mathrm{a}$ & Full Sodium & $5.88 \pm 1.719 \mathrm{a}$ & Full Sodium & $6.09 \pm 1.574 \mathrm{a}$ \\
\hline Salona* & $4.88 \pm 1.874 \mathrm{~b}$ & Salona* & $4.95 \pm 1.902 \mathrm{~b}$ & Salona* & $4.93 \pm 1.639 \mathrm{~b}$ \\
\hline Alta* $^{*}$ & $4.49 \pm 1.965 \mathrm{~b}$ & Alta* $^{*}$ & $4.76 \pm 2.125 \mathrm{~b}$ & Alta* & $4.77 \pm 1.888 \mathrm{~b}$ \\
\hline $\mathrm{NaCl/KCl} \mathrm{blend*}$ & $4.36 \pm 1.795 \mathrm{~b}$ & NaCl/KCl blend* & $4.44 \pm 1.878 \mathrm{~b}$ & NaCl/KCl blend* & $4.48 \pm 1.574 \mathrm{~b}$ \\
\hline Low Sodium & $3.31 \pm 1.671 \mathrm{c}$ & Low Sodium & $3.39 \pm 1.685 \mathrm{c}$ & Low Sodium & $3.60 \pm 1.71 \mathrm{c}$ \\
\hline Low Sodium* & $3.18 \pm 1.569 \mathrm{c}$ & Low Sodium* & $3.17 \pm 1.547 \mathrm{c}$ & Low Sodium* & $3.53 \pm 1.639 \mathrm{c}$ \\
\hline
\end{tabular}

The Friedman's analysis of ranks indicated a significant difference in ranking scores $\left(\right.$ Chi-Sq $\left.{ }_{5}=143.38, \mathrm{P}<0.0001\right)$ when comparing all variables, and Tukey's HSD at $5 \%$ significance was used for the post hoc analysis (Table 3.13).

Table 3.13. Bacteria Cultured Ranking Scores Post Hoc Analysis. Tukey's HSD at 5\% was use for post hoc analysis; samples with different letter differences are significantly different $(\alpha=0.05)$ from one another. A higher mean score indicates a lower liking, while a lower mean indicates a higher liking. An * indicates the sample was dipped in $0.275 \%$ SEA-i solution prior to testing.

\begin{tabular}{|c|c|c|}
\hline \multicolumn{2}{|c|}{ Bacteria Cultured Ranking Scores - Post Hoc Analysis } \\
\hline Sample & Mean & Differences \\
\hline Low Sodium* & 475.00 & A \\
\hline Low Sodium & 420.00 & A B \\
\hline NaC1/KClblend* & 352.00 & B C \\
\hline Alta (2345)* & 333.00 & C \\
\hline Salona* & 284.00 & $\mathrm{C}$ \\
\hline Full Sodium & 194.00 & $\mathrm{D}$ \\
\hline
\end{tabular}


The ranking scores among the six variables of bacteria cultured cheese yielded similar results to those found in the hedonic testing. The full sodium cheese had the lowest mean ranking score and thus was preferred more than the other cheeses, while the low sodium (dipped and non-dipped) cheeses were preferred the least. The Salona, $\mathrm{ALTA}^{\mathrm{TM}}$, and $\mathrm{NaCl} / \mathrm{KCl}$ blend were not significantly different in preference; additionally the $\mathrm{NaCl} / \mathrm{KCl}$ blend was not significantly different from the low sodium (non-dipped) cheese. The non-dipped low sodium cheese was not significantly different from the dipped low sodium cheese for ranking preference.

When comparing the six variables the full sodium cheese was consistently liked and preferred the most, the $\mathrm{NaCl} / \mathrm{KCl}$ blend, Salona, and $\mathrm{ALTA}^{\mathrm{TM}}$ were consistently not significantly different from one another in liking and preference, and the low sodium (dipped and non-dipped) cheeses were typically not significantly different from each other and were liked and preferred the least.

\subsection{Discussion}

Decreasing sodium in cheese is a major challenge, reducing the sodium in mozzarella cheese is especially difficult due to the naturally bland flavor of mozzarella. A series of affective and discrimination sensory tests were employed to understand the effect of reducing sodium in mozzarella cheese. Due to the complex role sodium plays in cheese, the sensory testing was implemented in two phases: 1) antimicrobial testing, and 2) salt replacer testing, to understand these effects separately. 


\subsubsection{Antimicrobial Sensory Testing}

One of sodium's major roles in cheese is inhibition of microbial growth through decreasing water activity (Guinee, 2004); with decreasing sodium levels in cheese an additional antimicrobial needs to be incorporated into the modified cheese matrix to maintain quality. The first triangle tests were performed on mozzarella cheese with typical sodium levels (1.8\%) while only testing two antimicrobials at each session. These tests provided a preliminary understanding of how the antimicrobials were perceived by the consumers; however, they did not provide a clear understanding of how the antimicrobials were perceived during the same session by the same consumers. After testing, the commercial/typically consumed sodium level in the test cheeses were hypothesized to perhaps be interfering with the flavors of the antimicrobials, by potentially masking off-flavors. Despite the initial tests having limitations, antimicrobials in full and low sodium cheese are still useful and of importance; the data suggested further investigation of NovaGard CB1 considering consumer difficulty of detecting this antimicrobial when compared to the non-dipped cheeses (Table 3.3).

The subsequent triangle tests were performed on lower sodium mozzarella cheese $(0.86 \%)$ which was thought to simplify the flavor of the cheese and antimicrobial, and would provide a clearer understanding of the consumer acceptability of these antimicrobials with lower sodium mozzarella cheese. These triangle tests required consumers to discriminate between all of the antimicrobial dipped cheeses compared to non-dipped cheeses in each session; thus, providing additional information on how the antimicrobials compared in terms of sensory transparency. Although no significant differences were observed between the antimicrobials, the results suggested further 
investigation of Protect-M and SEA-i F75 due to the number of incorrect answers when attempting to discriminate between dipped and non-dipped cheeses (Tables 3.4 and 3.5). These two triangles tests provided further insight into the use of these antimicrobials on lower sodium cheeses and with consumers of mozzarella cheese. Consumers are thought to be more effective at discriminating between product differences compared to nonproduct consumers (Stone et al., 2012); despite the relatively low sample numbers ( $\mathrm{n}=24$ and 33) the data is thought to be more accurate when using mozzarella consumers. Additional investigation of Protect-M and SEA-i F75 was clearer from the results of these triangle tests.

Considering the debate between whether triangle tests or duo-trio tests are more effective at determining differences between products (Meilgaard et al., 2007; Stone et al., 2012) two duo-trio tests were performed to test the four antimicrobials in the same session compared to non-dipped cheese with consumers and compared these findings to those of the previous triangle tests. The results from the duo-trio tests differed slightly from those of the triangle tests, with consumers having greater difficulty identifying the sample which was different from the reference in the PuraQ and SEA-i F75 samples (Table 3.6). In the second duo-trio test, consumers had greater difficulty identify the different sample for the SEA-i F75 and NovaGard CB1 samples, although there were no significant differences in either the first or second duo-trio test sessions (Table 3.7).

Whether the duo-trio test was more or less powerful is still unclear, but it did provide an additional review of the samples and the SEA-i F75 antimicrobial solution was the most consistently difficult sample for consumers to detect from the non-dipped cheeses compared to the other antimicrobials. However, increasing the number of subjects would 
increase power, and the potential to see differences between samples, but due to the consumer's consistent inability to detect SEA-i F75 dipped cheeses compared to nondipped cheeses, it was recommended at the tested concentration of $0.275 \%$ for further sensory testing with the salt replacers.

After discrimination testing was completed, a hedonic test was performed to ensure one antimicrobial was not liked significantly more than the others. The only significant difference found between the four antimicrobials and a control was in aroma liking, where the aroma of SEA-i F75 was liked significantly more than the aroma of NovaGard CB1 (Table 3.8). These findings were useful for validation of selecting SEA-i F75 as the antimicrobial to be tested with the salt replacers. Considering these antimicrobials are thought to be relatively sensory transparent, it is slightly surprising one would be liked more than another; however, one theory for explaining this could be SEAi F75 was the most liked because it had suggested through the series of discrimination tests to be transparent while the other antimicrobials did not yield the same results. Considering SEA-i F75's transparency, perhaps SEA-i F75 has no aroma was liked more than the potentially foul aromas of the other samples or possibly had a pleasant aroma and thus liked more than the others. Regardless, the hedonic test further validated the use of SEA-i F75 in sensory tests with salt replacers.

The time and concentration test was developed to better understand what effects modifying dip time and solution concentration of the SEA-i F75 antimicrobial would have on difference detection between a dipped and non-dipped cheese. The results of the time and concentration sensory test indicated either increasing the time in solution from 2 to 3 minutes or increasing the solution concentration by fifty percent could not be 
detected by the consumers; however, when both the solution dip time and concentration were increased, the consumers were able to detect a difference between those dipped cheeses and the non-dipped cheeses (Table 3.9). Increasing either dip time or solution concentration had no apparent effect on the discrimination between the dipped and nondipped cheeses; this information is useful for recommended use and processing specifications when manufacturing a low sodium mozzarella cheese.

\subsubsection{Salt Replacer Sensory Testing}

Both direct acidification and bacteria cultured acidification methods are used by industry manufacturers when making mozzarella cheese. Both methods were tested with the SEA-i F75 antimicrobial and salt replacers to make a recommendation for a given manufacturer (using direct or bacteria acidification) for low sodium mozzarella cheese. Considering a salt replacer is expected to elicit flavor in a low sodium mozzarella cheese, there was no need to perform discrimination testing with the salt replacer variables because there would be obvious and expected differences between a cheese containing salt replacers and a cheese without salt replacers. The main objective of the salt replacers sensory testing was simply to determine which of the salt replacers was the most liked by subjects. Considering the test was an affective test the use of consumers alone was not necessary, both consumers and non-users of mozzarella cheese were used in this testing.

The results of both the direct acidification cheese and the bacteria cultured acidification cheeses had significantly different liking scores across the salt replacer variables. In both types of cheeses, the full sodium cheese was liked and preferred the most while the low sodium cheeses (dipped and non-dipped) were consistently liked and 
preferred the least; both of these results were expected. Note, the highest hedonic scores of the full sodium cheese were just above 5.0 which is considered "neither like nor dislike" on the hedonic scale, all other samples ranked below 5.0, and thus considered "disliked" samples. The cheese used for the hedonic sensory testing was produced five days prior to sensory testing, this "new" cheese did not have time to rest and equilibrate with the salt replacers before testing, which could be one explanation for why the hedonic scores were lower than expected. In both cheese types, the $\mathrm{NaCl} / \mathrm{KCl}$ blend, Salona, and ALTA $^{\mathrm{TM}}$, typically were not significantly different from one another in liking or preference ranking scores; however, in the direct acidified cheese the $\mathrm{NaCl} / \mathrm{KCl}$ blend was liked (non-significantly) more than the Salona and ALTA ${ }^{\mathrm{TM}}$ cheeses, while in the bacteria cultured cheese the Salona was liked (non-significantly) more than the ALTA ${ }^{\mathrm{TM}}$ and $\mathrm{NaCl} / \mathrm{KCl}$ blend. Due to the apparent similarities between the $\mathrm{NaCl} / \mathrm{KCl}$ blend, Salona, and ALTA ${ }^{\mathrm{TM}}$ salt replacers in mozzarella cheese, the most appropriate salt replacer appears to be relative. If a manufacturer produces mozzarella cheese made with bacteria cultures, perhaps the best replacer is Salona, while if the cheese is made by direct acidification perhaps the manufacturer should use the $\mathrm{NaCl} / \mathrm{KCl}$ blend.

There were no significant differences found between the three salt replacers in either type of cheese, therefore statistically speaking the three replacers are interchangeable. However, if one salt replacer was far more inexpensive (i.e. $\mathrm{NaCl} / \mathrm{KCl}$ blend) than the other options, perhaps the cheaper product should be considered first. Additionally, more literature and information is available regarding the use of a $\mathrm{NaCl} / \mathrm{KCl}$ blend in cheeses (El-Bakry et al., 2011; Cruz et al., 2011; Guinee, 2004; Grummer et al., 2012; Katsaiari et al., 1997; Ayyash \& Shah, 2010) compared to other 
salt replacers, especially Salona and ALTA ${ }^{\mathrm{TM}}$. From a health perspective, increased consumption of potassium can elicit a positive health effect on those with sodiuminduced hypertension (Katsiari et al., 1997), manufacturers can use the information available in the literature to their advantage by working with $\mathrm{NaCl} / \mathrm{KCl}$ to find the blend ratio which works best in their specific cheese matrix. The $\mathrm{NaCl} / \mathrm{KCl}$ blend used for this study was approximately a 1:1 ratio, other studies have found a 1:1 ratio to be an effective replacement in cheese (Cruz et al., 2011; Katsiari, 1997) while others suggest a slightly lower ratio may decrease bitterness (Grummer et al., 2012).

Based upon these series of sensory tests $\mathrm{NaCl} / \mathrm{KCl}$ blend, Salona, or ALTA ${ }^{\mathrm{TM}}$ would be appropriate salt replacers to use in a low sodium mozzarella cheese and dipped in a $0.275 \%$ solution of SEA-i F75 for two minutes; however, the $\mathrm{NaCl} / \mathrm{KCl}$ blend appears to have slightly more benefits. Although this study provided a series of liking and discrimination testing, valuable information could be obtained from broader consumer testing or descriptive analysis of the different cheeses to determine drivers of liking, and degree of difference from a control or full sodium mozzarella cheese sample.

\subsection{Conclusion}

Sodium reduction has been a major challenge for a variety of food manufacturers, and especially difficult for mozzarella cheese manufacturers due to the naturally bland flavor of mozzarella. Reducing sodium content in a variety of cheeses has been studied, but little information is available regarding the effect of lowering sodium in LMPS mozzarella cheese. This study performed a series of affective and discrimination sensory tests to identify an effective and sensory transparent antimicrobial. After selecting an 
appropriate antimicrobial (SEA-i F75), hedonic testing was implemented to determine which of three specific salt replacers was liked best by subjects in mozzarella cheese dipped in the selected antimicrobial. Although the three salt replacers were not significantly different for hedonic or ranking scores, certain salt replacers may be advantageous in certain types of mozzarella cheese or in certain circumstances. Additional sensory testing, including larger consumer tests and descriptive analysis, should be performed with the flavor enhancers to better understand key differences between the samples, and eventually aid in selection of the best salt replacer for a given cheese or manufacturer. These preliminary sensory studies provide new insight into options for reducing sodium in mozzarella cheese. 


\title{
$\underline{4.0}$
}

\section{Development of a Novel Method for Evaluating Emotions and Texture:}

\section{Imagery versus Language}

\begin{abstract}
Could images be the next step to understanding consumers' attitudes toward texture and emotion? Little work has been conducted to evaluate the relationship between texture and its emotional response with consumers. The objectives of this study were: 1) to investigate the use of two texture assessment methods across three different food categories and 25 specific texture attributes, 2) evaluate the effectiveness of three emotion methods, 3) to measure change in emotional responses throughout the consumption experience, and 4) assess both the changes in emotion and texture attributes.

Subjects were randomly selected to use one of two emotion image methods or an emotion words method and either the texture image cards or texture words only. Consumer selected emotion images were less variable than emotion words in a positive emotion. Differences between the two texture methods occurred among samples tested on the first day of testing, but not for those tested on the second and third day. Identifying the variability between the emotions allows researchers to more consistently link emotion and texture methodology to the consumer's change in emotion and perceived textural changes perceived over the consumption experience.
\end{abstract}


Highlights: >Novel methods for evaluating emotions and texture were developed and tested. > Higher variability in a positive emotion was found in the words only emotion testing method. > Greater variability in a negative emotion was found in the My Pictures emotion testing method. >Differences in change in texture attributes between texture testing methods. >Careful selections of images for the texture cards should be implemented to increase consistency amongst subjects.

Keywords: texture, emotion, alternative methodology, imagery, consumer perception, IMET 


\subsection{Introduction}

Textural characteristics, in addition to appearance and flavor comprise the primary sensory elements which determine food acceptability by consumers (Bourne, 1978) and the importance of texture analysis as it relates to sensory assessments has been well documented (Civille \& Liska, 1974; Brandt et al., 1962; Pons \& Fiszman, 1996; Breene, 1975). A variety of different texture methods developed to correlate instrumental texture measurements to sensory texture assessments exist, including: Texture profile analysis (TPA), temporal dominance of sensation (TDS), jaw-muscle EMGs, and dynamic mechanical analysis (DMA) to name a few (Bourne, 1978; Foegeding et al., 2011; Anker et al., 1999). Many studies to date have used the TPA method for texture analysis (Ares et al., 2006; Breene, 1975; El-Bakry et al., 2011; Pons \& Fiszman, 1996) with the key advantage of TPA being the suggested correlation between the instrument texture measurements and sensory texture assessments (Bourne, 1978; Pons \& Fiszman, 1996). Despite this advantage, other texture methods take into account the physiological and psychophysical elements of texture in addition to the mechanical and physical aspects present in TPA (Foegeding et al., 2011), thus providing texture assessments which are closer to consumer perceptions of food textures. The aim of consumer texture perception methods provides the opportunity to develop alternative methods of texture analysis measurements.

Traditionally, texture measurements are correlated with descriptive sensory texture assessments; however, they do not necessarily correlate with the consumer's textural perception of the product (Foegeding et al., 2011; Moskowitz, 1987). Consumers are typically not asked to perform highly specific texture attribute questions because they 
are not trained in how to characterize these textures (Moskowitz, 1987). Yet, if the consumers were given images which depicted these specific texture attributes perhaps two key outcomes could be obtained: 1) additional textural attributes could be assessed above what is currently available through TPA (fracturability, hardness, cohesiveness, adhesiveness, springiness, gumminess, and chewiness) and other methods (Pons \& Fiszman, 1996; Bourne, 1978) and 2) capture specific textural characteristics of food products as perceived directly from the consumers themselves. Texture is a multimodal characteristic of food (Foegeding et al., 2011) and several successful approaches have been developed to obtain texture attributes; however, none of the current texture methods utilize texture images and consumer perceptions to help understand texture characteristics.

In addition to consumer perception of texture characteristics, perhaps the development of alternative texture methods may go beyond texture liking and rating. Rolls and Grabenhorst (2008) suggests emotions and oral texture are both processed in the orbitofrontal cortex (OFC) of the brain and perhaps combining emotion and texture methods may yield novel information about both fields. Emotion testing with flavor and aroma has been well studied (Seo et al., 2009; Macht, 1999; Thomson \& Crocker, 2011; Croy et al., 2011; Desmet \& Schifferstein, 2008; Macht, 2008; King \& Meiselman, 2010; King et al., 2010), but little investigation has been done on the potential relationship between texture and emotion in the context of eating.

One caveat of measuring emotion is the non-cognitive nature of emotions which can be difficult to measure and quantify. Additionally, one criticism of current emotion testing is the potential for variability from person to person, again an intrinsic 
characteristic of emotions (Schifferstein \& Desmet, 2010). Thomson et al. (2010) mentions the difficulty of using words in emotion testing due to the issues of scaling and the rational thought processes that occur with words and scaling. Emotions and picture images are thought to be more effectively processed in the brain's right cerebral hemisphere, while language and logic are thought to be better processed in the left hemisphere (Freberg, 2009), although this is an over simplification (Sander, 2012); however, exploration of this idea could shed light on opportunities for alternative emotion testing methods. Furthermore, Croy et al. (2011) investigated the use of alternative emotion evoking stimuli through visual and olfactory stimuli versus no stimuli and found visual stimuli was better at evoking negative emotions than olfactory stimuli; overall, either stimulus was better than no stimulus at evoking emotion. The opportunity to develop an alternative emotion and texture measurement method exists considering the difficulties in understanding consumer acceptance of specific texture attributes and the inherit difficulties of measuring emotions with consumers. Given the apparent relationship between emotion and texture (Rolls \& Grabenhorst, 2008) as well as emotion and imagery (Freberg, 2009), perhaps the use of images for eliciting emotions and to visually explain texture attributes could be provided to consumers to obtain novel information about both emotions and texture as perceived by consumers.

Additionally, the relationship between gender and emotion will be explored considering general social convention suggests that women are "more emotional" than men (Gard \& Kring, 2007) and extensive data indicates women may actually be more emotionally expressive than men (Brody, 1997). Other studies have found significant differences in emotional expressions or responses between males and females (Gard \& 
Kring, 2007; Kring \& Gordon, 1998; Macht, 1999; Seo et al., 2009). Studies comparing the relationship between emotion and gender/sex differences in the context of food are often conducted only with abnormal eating behaviors (Costanzo et al., 1999; Macht et al., 2003). Previous studies suggest an underlying difference in regard to emotions between males and females (King et al., 2010; Kring \& Gordon, 1998; Seo et al., 2009; Macht, 1999; Costanzo et al., 1999); this study anticipates finding a significant difference between sexes. Additionally, Kring and Gordon (1998) suggest the frequency or intensity of positive and negative emotional experiences are manifested more in women; similar findings are anticipated for this emotional comparison between men and women.

The use of emotion images is thought to be more effective at capturing consumer's emotional responses to food products rather than words alone by decreasing movement between the two cerebral hemispheres. The use of personal emotion evoking images and predefined emotion images will be investigated to understand variability in emotions. The effectiveness of these methods were tested by comparing emotional responses across three food product categories and in two geographic locations. This study used three different emotion testing methods (two image related, and one word) to determine: 1) whether the testing methods yield significantly different emotion results and, 2) which methods provide the most consistent (least variable) results. An image related emotion testing method was hypothesized to have significantly different changes in emotion and less variability than the words only method. Additionally, changes in emotion were compared between males and females to observe any differences between genders. To investigate the effectiveness of images for texture attribute testing, two texture methods were used: 1) texture words, and 2) texture images. The texture images 
were provided by the investigators for testing and will contain 25 unique texture attributes. The objectives for understanding texture the effectiveness of the texture methods among consumers were to: 1) provide consumers with texture images cards to identify specific texture attributes of three food categories, 2) capture changes in specific texture attributes across the eating experience, and 3) observe potential relationships between the texture method used and the changes in texture attributes and emotions across the eating experience. It was hypothesized that through the use of texture images, smaller changes (decreased variability) in texture attribute responses would occur among consumers.

\subsection{Methodology}

Emotion testing was conducted across three days in two geographical locations (California Polytechnic State University - San Luis Obispo, and The Ohio State University). The four commercial carbonated orange soda products (Table 4.1) were tested on day one $(n=217)$. The six commercial dairy beverages (Table 4.2) were tested on day two $(n=219)$, and seven samples of commercial convenience cheese samples (Table 4.3) were tested on day three $(n=216)$. This protocol was approved by the California Polytechnic State University and The Ohio State University Institutional Review Board prior to testing.

\subsubsection{Subjects}

Subjects were recruited by university and public advertisements and email; subjects were selected based upon being consumers of the test products (consumed "everyday" to "about once a month") and non-users of Crest-Pro health products. Prior to 
testing, subjects were asked to complete: 1) a learning styles questionnaire and 2) their "My Pictures" poster board. The My Pictures poster board was divided into 12 boxes (one emotion listed per box) and subjects were asked to place meaningful pictures that represented the different emotions to themselves in each box. Subjects included students, university staff, and community members (20.9 \pm 3.56 years) with 159 females $(73 \%)$ and 60 males (23\%) and participants were financially compensated.

\subsubsection{Products}

A series of QMA/Napping exercises were performed to select the products for testing; commercial samples which elicited different textural experiences based upon defined axes were chosen for testing. The QMA/Napping exercised were performed separately for each of the different food product categories. The textural attributes chosen to distinguish the orange soda sample were amount of carbonation and bubble size, viscosity and mouth coating for the dairy beverages, and firmness and stringiness for the convenience cheeses.

Table 4.1. Orange Soda Samples
\begin{tabular}{c}
\hline $\begin{array}{c}\text { Orange Soda } \\
\text { Samples: }\end{array}$ \\
\hline Fanta \\
\hline Izzc \\
\hline $\begin{array}{c}\text { Orangina } \\
\text { Sunkist }\end{array}$ \\
\hline
\end{tabular}


Table 4.2. Dairy Beverage Samples

\begin{tabular}{|c|}
\hline Dairy Beverage Samples: \\
\hline Chug Chocolate Shake \\
\hline Danimals Strawberry Drinkable Yogurt \\
\hline Horizon Strawberry Milk \\
\hline Lifeway Strawberry Kefir \\
\hline Nesquick Low Fat Chocolate Milk \\
\hline Silk Dark Chocolate Almond Milk \\
\hline YooHoo Chocolate Drink \\
\hline
\end{tabular}

Table 4.3. Convenience Cheese Samples

\section{Convenience Cheese Samples:} Albertson's Regular String Cheese Frigo Light String Cheese Frigo Regular String Cheese Sargento Light String Cheese Sargento Reduced Fat CoJack Sargento Reduced Fat Sharp Cheddar

All samples were kept at refrigeration temperature $\left(37^{\circ} \mathrm{F}\right)$ until served to subjects; all samples were served directly out of the same refrigerator. The commercial product remained in its original container until the morning of testing, prior to pouring the commercial dairy beverage; containers were lightly shaken, and poured into two ounce soufflé cups. With the exception of Orangina, the carbonated orange soda were not agitated prior to serving and the convenience cheeses were cut in half and placed on labeled paper plates. Soufflé cups and paper plates were labeled with randomized 3-digit codes for serving. Subjects received approximately two fluid ounces of the orange soda beverages and dairy beverages, and half of a convenience cheese stick for testing which was served directly from refrigeration. A William's design was used for testing, where all samples were randomized within and across subjects and each subject saw all products tested on each day: four samples on day one, seven samples on day two, and six samples on day three. No replication was performed; each subject tested each product only once, with approximately 30 seconds between the end of one sample and the beginning of the next sample. The environmental conditions between the two locations differed slightly between the two locations. At the Ohio State University subjects performed each day of testing in temperature regulated sensory booths $\left(72^{\circ} \mathrm{F}\right)$ under incandescent spot lights, 
while at Cal Poly State University subjects completed the test in a large conference room $\left(70^{\circ} \mathrm{F}\right)$ with 14 subjects testing at a time, evenly spaced under fluorescent lighting.

\subsubsection{Emotion Methods}

Three different emotion methods were used for testing 1) words only, 2) Predefined pictures, and 3) My Pictures. The emotion method (My Pictures, Predefined pictures, and words) were randomly allocated to the subjects upon arrival to the testing sight; randomization occurred separately for each day of testing. The words only method was a control to compare the use of words during emotion testing to pictures during testing. With the words only method no visual aid was provided, only the words used on

the questionnaire were given. The emotion words were presented in choose-all-that-apply (CATA) format. The Predefined pictures method consisted of a poster board with 12 emotion images (Fig. 4.1); the investigators selected the images by consensus based upon universal images of emotions. All Predefined pictures poster boards were the exact same, with the same emotion word location and pictures, thus acting as a control between the two image-based methods. 


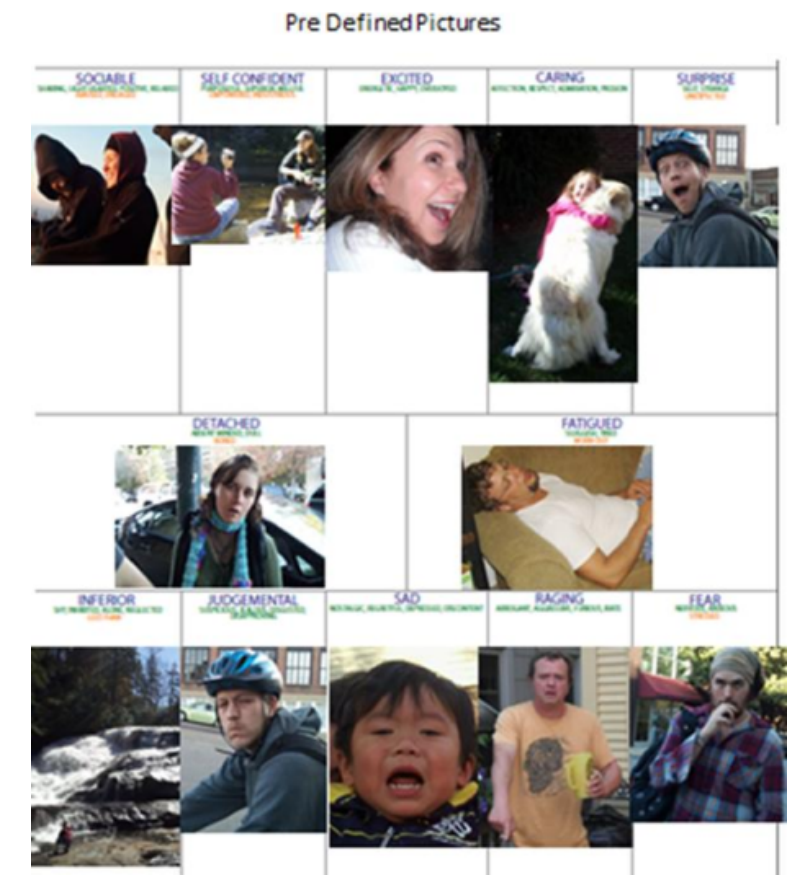

Fig. 4.1. The Predefined Pictures Method. Images for this method were selected by researchers and did not change.

The My Pictures method included the poster board the subject created and brought into testing (Fig. 4.2). Images used for the My Picture method could be from magazines, newspapers, the internet, or personal photos.

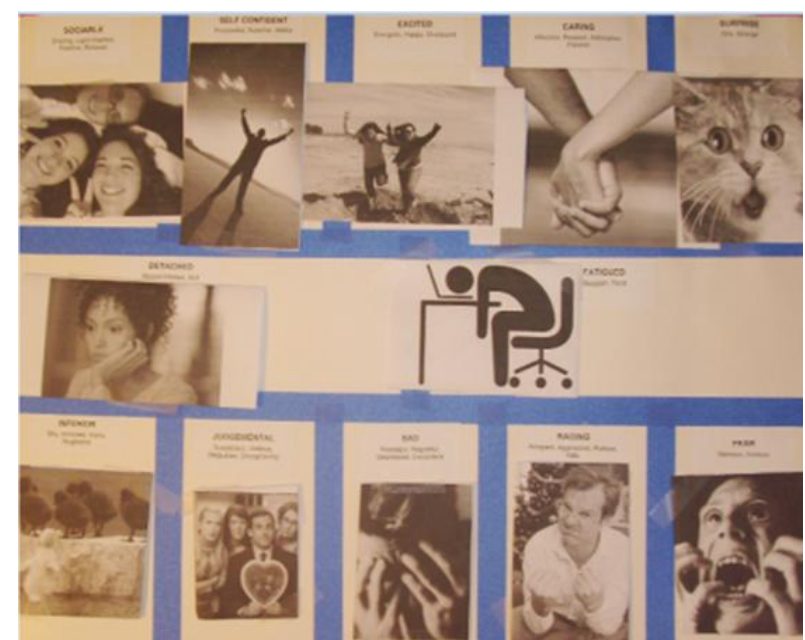

Fig. 4.2. Example of My Pictures Method. These poster boards were used by a subject during testing; images used varied by each subject. 


\subsubsection{Emotions for Testing}

The following twelve emotion words were selected for testing: sociable, selfconfident, excited, caring, surprise, detached, fatigued, inferior, judgmental, sad, raging, and fear. These emotion words were selected based upon research done by Thomson and Crocker (2011), where emotion testing was conducted across four countries with hundreds of emotion words. The twelve emotions selected were amongst those considered most suitable for emotion testing with consumers. For analytical purposes the first five emotions listed were considered "positive" emotions, the following two "neutral" emotions, and the last five "negative" emotions.

\subsubsection{Questionnaire}

Subjects were required to complete demographic questions before beginning the test including age, sex, and ethnicity. The questionnaire first asked for the subject's initial emotion(s) presented as CATA; the subject used their emotion method tool, based upon randomization, at this point. Next subjects were asked to rank 25 texture attribute questions (using designated texture method), overall liking, overall flavor liking, and overall texture liking at the first taste, halfway through, and at the end of consuming the product. Texture attribute questions were ranked using a categorical intensity scale, and a 9-point hedonic scale was used for all liking questions. Subjects then entered their emotional state (CATA) after consuming the product, and proceed to the next sample. Compusense ${ }^{\circledR}$ at-hand was used for data collection at both testing sites. 


\subsubsection{Texture Methods}

Two texture methods were used for testing: 1) texture cards, and 2) texture words. The texture cards consisted of 25 unique texture images which illustrated each texture attribute being tested (Table 4.4). Each texture attribute image card included: 1) the attribute name, 2) an overall image of the attribute, and 3) varying degrees of that texture (Fig. 4.3); researchers collaboratively developed the images used for the cards. The texture words method consisted of only the texture attributes presented on the questionnaire, no visual aid was provided. Texture methods were randomly allocated to each subject upon arrival to the testing site for each day of testing. The combination of the emotion measurement and texture measurement comprise the Image Measurement of Emotion and Texture (IMET) method.

Table 4.4. Texture Attributes. List of all texture attributes used for all three days of testing, texture attribute words were presented as shown above.

\begin{tabular}{|l|l|l|l|l|}
\hline \multicolumn{5}{|c|}{ Texture Attributes } \\
\hline Mouth Coating & Filling Composition & Skin/Shell & Soft & Hardness \\
\hline Carbonation & Shear & Surface Deviation & Blanket & Melt \\
\hline Break Resistance & Hollow Break & Grittiness & Sponginess & Creamy \\
\hline Bounciness & Resilience & Dissolve & Rubbery & Waxy \\
\hline Fibrousity & Stickiness & Crumble & Sandy & Bite Location/Place on Tongue \\
\hline
\end{tabular}

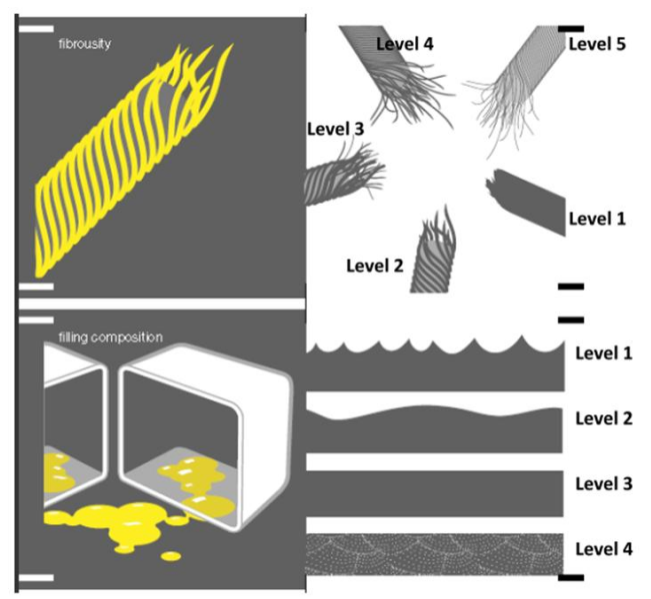

Fig. 4.3. Example of Texture Image Cards. Example texture image card used for testing includes "fibrousity" and "filling composition." 


\subsection{Data Analysis}

\subsubsection{Emotion Analysis}

The statistical analysis of the change in emotion was calculated by subtracting the end emotions from the beginning emotions; an oneway ANOVA comparison between each change in emotion by emotion method was performed using JMP (JMP, Version 10. SAS Institute Inc., Cary, NC). Although the emotional responses were captured as categorical, the data was treated as continuous in order to interpret the direction and intensity of the emotion changes and how that change related to the different emotion types (positive, neutral, and negative) by each product. Considering the emotion data was analyzed as continuous after being captured as categorical, the Kruskal-Wallis rank sum test was used for analysis to help aid with issues of non-normality in the data. If a significant Kruskal-Wallis rank sum p-value occurred $(\alpha=0.05)$, the Steel-Dwass all pairs nonparametric post hoc comparison was performed to determine which methods were significantly different from one another $(\alpha=0.05)$. Additionally, in order to understand the equality of variances between the three testing methods, an unequal variance test was performed, and the Brown-Forsythe's test was used to determine equality of variance; this test is more robust to non-normality compared to the more popular Levene's test.

When considering the changes in emotion by gender, an overall MANOVA comparing all changes in emotion by gender was performed; given a significant whole model $(\alpha=0.05)$ further investigation was carried out to determine which changes in emotion differed by gender. Considering the changes in emotion were treated as 
continuous, the Wilcoxon rank test was used for determining significant differences $(\alpha=0.05)$ among the changes in emotion by gender ANOVAs.

\subsubsection{Texture Analysis}

For texture analysis, a MANOVA was performed comparing the change in texture attributes by texture method for orange soda only, dairy beverage only, cheese only, and all data combined. If a significant whole model effect existed, then each texture attribute was compared separately by way of a pooled t-test. Additionally, oneway ANOVAs were performed on the changes in emotion by texture method (words and cards) for each food product category. The Kruskal-Wallis rank sum test was used for analysis $(\alpha=0.05)$, if a significant $p$-value was determined, the Steel-Dwass all pairs nonparametric post hoc comparison was performed to determine which emotions were significantly different $(\alpha=0.05)$; all analysis was performed using JMP (JMP, Version 10. SAS Institute Inc., Cary, NC).

\subsection{Results}

\subsubsection{Emotion methods and each emotion}

When comparing each change in emotion by emotion testing method, significant differences between emotion methods only appeared in the positive emotions: caring $($ Chi-Sq $2=8.7333, \mathrm{P}=0.0127)($ Table 4.5), sociable $($ Chi-Sq $2=8.4442, \mathrm{P}=0.0147)($ Table 4.6), and self-confident $\left(\mathrm{Chi}-\mathrm{Sq}_{2}=7.7391, \mathrm{P}=0.0209\right)$ (Table 4.7). All other comparisons between each change in emotion and emotion testing method were not significant $(\alpha=0.05)$. Fig. 4.4 provides general comparisons between the changes in emotion by type of emotion (positive, neutral, and negative) and emotion testing methods. Among the 
significant differences (caring, sociable, and self-confident), the words only method had significantly greater decreases in changes in emotion compared to the My Pictures emotion testing method (Fig. 4.4; Table 4.5 - 4.7).

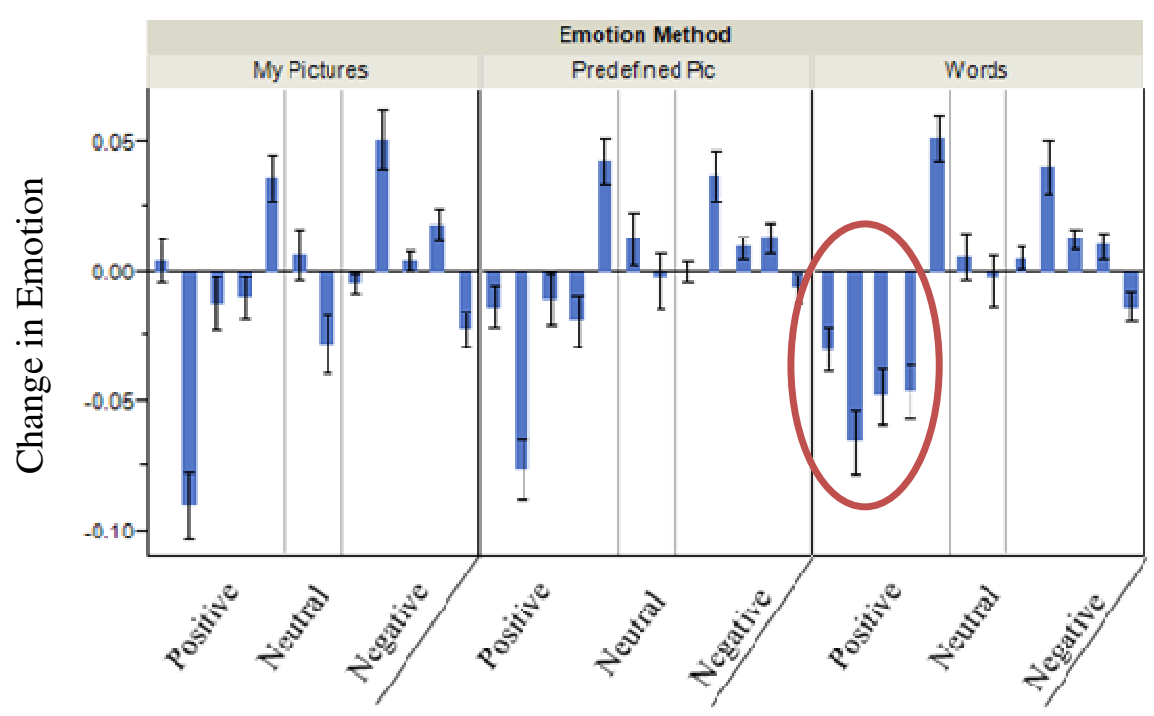

Fig. 4.4. Change in Emotion by Emotion Type and Method. The effectiveness of emotion method appears to vary by emotion type.

The Predefined pictures (Fig. 4.1) and words only methods did not differ significantly for change in caring (Table 4.5) or change in self-confident (Table 4.7); however, they did differ for change in sociable (Table 4.6).

Table 4.5. Change in Caring by Emotion Method. Nonparametric post hoc comparison comparing change in caring across the three testing methods; including an adjusted mean value for consideration given the use of the Kruskal-Wallis test.

\begin{tabular}{|c|c|c|c|l|}
\hline \multicolumn{5}{|c|}{ Change in Caring by Emotion Method } \\
\hline Emotion Method & Mean & (Mean-Mean0)/Std0 & Std Error & Differences \\
\hline My Pictures & 0.0040 & 2.598 & 0.00815 & A \\
\hline Predefined Pic & -0.0139 & -0.085 & 0.00822 & A $\quad$ B \\
\hline Words & -0.0305 & -2.522 & 0.00821 & B \\
\hline
\end{tabular}


Table 4.6. Change in Sociable by Emotion Method. Nonparametric post hoc comparison comparing change in sociable across the three testing methods; including an adjusted mean value for consideration given the use of the Kruskal-Wallis test.

\begin{tabular}{|c|c|c|c|c|}
\hline \multicolumn{6}{|c|}{ Change in Sociable by Emotion Method } \\
\hline Emotion Method & Mean & (Mean-Mean0)/Std0 & Std Error & Differences \\
\hline Predefined Pic & -0.0106 & 1.523 & 0.01060 & A \\
\hline My Pictures & -0.0121 & 1.377 & 0.01052 & A \\
\hline Words & -0.0489 & -2.904 & 0.01059 & B \\
\hline
\end{tabular}

Table 4.7. Change in Self Confident by Emotion Method. Nonparametric post hoc comparison comparing change in self-confident across the three testing methods; including an adjusted mean value for consideration given the use of the Kruskal-Wallis test.

\begin{tabular}{|c|c|c|c|c|}
\hline \multicolumn{5}{|c|}{ Change in Self Confident by Emotion Method } \\
\hline Emotion Method & Mean & (Mean-Mean0)/Std0 & Std Error & Differences \\
\hline My Pictures & -0.0104 & 1.962 & 0.00954 & A \\
\hline Predefined Pic & -0.0196 & 0.722 & 0.00962 & A \\
\hline Words & -0.0465 & -2.691 & 0.00961 & B \\
\hline
\end{tabular}

The Brown-Forsythe's test indicated that the words only method had significantly $(\alpha=0.01)$ greater variability in self-confident $\left(F_{2,3694}=5.1309, P=0.0060\right)$. The Predefined pictures method had significantly greater variability in self-confident $\left(\mathrm{F}_{2,3694}\right.$ $=5.1309, \mathrm{P}=0.0060)$, and the My Pictures method had significantly greater variability in judgmental $\left(\mathrm{F}_{2,3694}=8.0071, \mathrm{P}=0.0003\right)$.

The MANOVA comparison across all changes in emotion by gender provided a significant whole model effect $\left(\mathrm{F}_{12,3684}=1.9418, \mathrm{P}=0.0256\right)$ with caring, sociable, and sad requiring additional investigation. The Wilcoxon rank test also indicated the only significant differences between changes in emotion and gender existing in caring (Chi-Sq $\left.{ }_{1}=8.2749, \mathrm{P}=0.0040\right)$, sociable $\left(\mathrm{Chi}_{-\mathrm{Sq}}{ }_{1}=4.3641, \mathrm{P}=0.0367\right)$, and sad $\left(\mathrm{Chi}-\mathrm{Sq}_{1}=\right.$ 4.4674, $\mathrm{P}=0.0345)$; tables $4.8,4.9$, and 4.10 contain the means, adjusted means, and standard errors for males and females for caring, sociable, and sad, respectively. 
Table 4.8. Change in Caring by Gender. Nonparametric comparison comparing change in caring by gender; including an adjusted mean value for consideration given the use of the Wilcoxon test.

\begin{tabular}{|c|c|c|c|}
\hline \multicolumn{4}{|c|}{ Change in Caring by Gender } \\
\hline Gender & Mean & (Mean-Mean0)/Std0 & Std Error \\
\hline Male & 0.00886 & 2.877 & 0.00902 \\
\hline Female & -0.02163 & -2.877 & 0.00555 \\
\hline
\end{tabular}

Table 4.9. Change in Sociable by Gender. Nonparametric comparison comparing change in sociable by gender; including an adjusted mean value for consideration given the use of the Wilcoxon test.

\begin{tabular}{|c|c|c|c|}
\hline \multicolumn{4}{|c|}{ Change in Sociable by Gender } \\
\hline Gender & Mean & $($ Mean-Mean0)/Std0 & Std Error \\
\hline Male & -0.00295 & 2.089 & 0.01165 \\
\hline Female & -0.03170 & -2.089 & 0.00717 \\
\hline
\end{tabular}

Table 4.10. Change in Sad by Gender. Nonparametric comparison comparing change in sad by gender; including an adjusted mean value for consideration given the use of the Wilcoxon test.

\begin{tabular}{|c|c|c|c|}
\hline \multicolumn{4}{|c|}{ Change in Sad by Gender } \\
\hline Gender & Mean & (Mean-Mean0)/Std0 & Std Error \\
\hline Male & 0.02461 & 2.114 & 0.00618 \\
\hline Female & 0.00933 & -2.114 & 0.00380 \\
\hline
\end{tabular}

Additionally, investigation of the individual subject's My Pictures poster boards suggested consumers were more likely to use images of themselves for the positive emotions and images of others for the negative emotions. The images used also suggested the use of universal images, similar across many poster boards, and individual images, unique images which would not necessarily elicit a certain emotion without a given context. The role of personal pictures and pictures of others, and universal and individual pictures may play a large role in understanding the effectiveness of the developed method. 


\subsubsection{Change in texture attributes and emotions by texture methods}

The MANOVA results for all the data combined found a significant difference across all changes in texture attributes by texture method $\left(\mathrm{F}_{25,3643}=1.7163, \mathrm{P}=0.0148\right)$. When testing each texture attribute for significance between the two methods, grittiness $\left(\mathrm{F}_{1,3667}=4.0449, \mathrm{P}=0.0444\right)$ and waxy $\left(\mathrm{F}_{1,3667}=8.4091, \mathrm{P}=0.0038\right)$ were significantly different between texture cards and texture words. The orange soda only overall texture method MANOVA was significant when comparing all changes in texture between the two texture methods $\left(\mathrm{F}_{25,842}=2.1395, \mathrm{P}=0.0010\right)$; the significant specific attributes included: stickiness $\left(\mathrm{F}_{1,866}=6.7688, \mathrm{P}=0.0094\right)$, surface deviation $\left(\mathrm{F}_{1,866}=4.2179\right.$, $\mathrm{P}=0.0403)$, crumble $\left(\mathrm{F}_{1,866}=6.7630, \mathrm{P}=0.0095\right)$, creamy $\left(\mathrm{F}_{1,866}=4.7922, \mathrm{P}=0.0289\right)$, waxy $\left(\mathrm{F}_{1,866}=13.1322, \mathrm{P}=0.0003\right)$, and place on tongue $\left(\mathrm{F}_{1,866}=5.2248, \mathrm{P}=0.0225\right)$. The MANOVA whole model results for change in texture attributes by texture method for both dairy beverages and cheese were insignificant $(\mathrm{P}=0.1781$ and $\mathrm{P}=0.2113$, respectively).

\subsubsection{Texture methods and change in emotion}

The results of the oneway ANOVAs for each change in emotion by texture method indicated differences between the texture cards and words across each of the product categories separately and combined. Significant differences between the changes in emotions by both texture methods exist for: orange soda (cards: Chi-Sq ${ }_{12}=116.54$, $\mathrm{P}<0.0001$; words: Chi-Sq $12=138.28, \mathrm{P}<0.0001$ ), dairy beverages (cards: Chi-Sq $12=$ 61.06, $\mathrm{P}<0.0001$; words: Chi-Sq $12=106.51, \mathrm{P}<0.0001$ ), and convenience cheeses (cards: 
Chi-Sq $12=42.92, \mathrm{P}<0.0001$; words: Chi-Sq $12=76.27, \mathrm{P}<0.0001$ ), as well as for all product categories combined (cards: Chi-Sq $12=184.59, \mathrm{P}<0.0001$; words: Chi-Sq 12 $=291.54, \mathrm{P}<0.0001)$. The Steel-Dwass all pairs nonparametric post hoc comparisons between each of the changes in emotion by texture indicated the texture words method consistently resulted in more significant differences between the tested emotions compared to the texture cards method for orange soda, dairy beverages, convenience cheeses, and all products combined (Table 4.11). Additionally, pooled t-test comparisons between each change in emotion by texture method suggested no significant difference ( $\alpha=0.05$ ) between the changes in emotion when using texture cards compared to using texture words. Fig. 4.5 provides a general visual comparison between the changes in emotion within each texture method (cards and words) across all product categories.

Table 4.11. Number of Post Hoc Significant Differences. Post hoc comparison of the change in emotion by both texture methods separately; texture words had more significant differences between emotions compared to the texture cards.

\begin{tabular}{|c|c|c|}
\hline \multicolumn{3}{|c|}{ Number of Post Hoc Significant Differences } \\
\hline Comparison & Texture Cards & Texture Words \\
\hline Orange Soda & 4 & 9 \\
\hline Diary Beverages & 11 & 20 \\
\hline Convenience Cheeses & 23 & 25 \\
\hline All Samples Combined & 34 & 41 \\
\hline
\end{tabular}




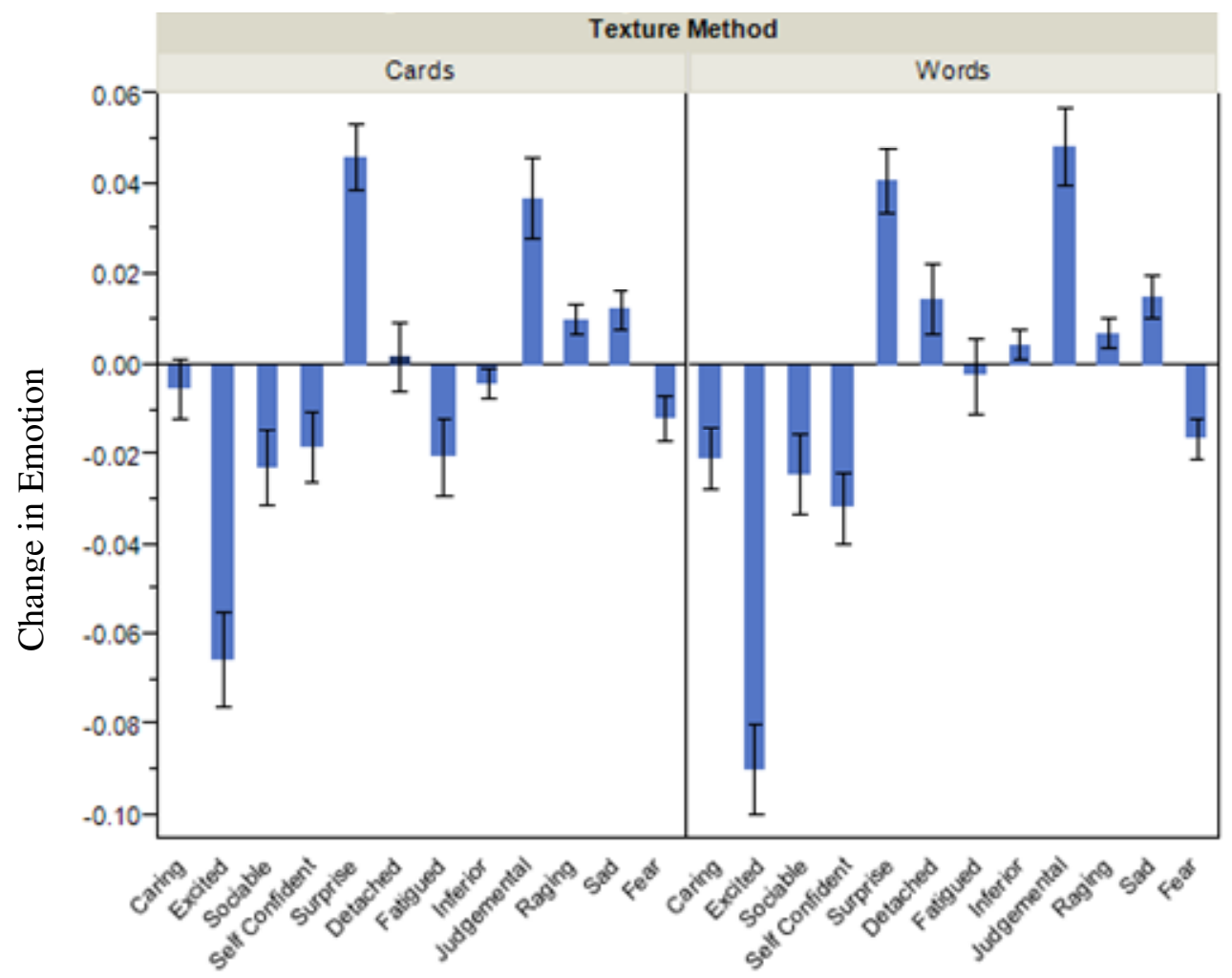

Fig. 4.5. Change in Emotion by Emotion and Method. Comparison of the two texture methods based upon the change in emotion for each emotion tested.

\subsection{Discussion}

\subsubsection{Image Measurement of Emotion and Texture}

The Image Measurement of Emotion and Texture (IMET) method was developed and implemented for this study. The IMET method utilizes images rather than words to guide consumers through changes in emotion testing and specific texture attribute scoring. Although this study employed the use of both consumer and researcher generated images for emotion testing, the IMET method only requires the use of consumer selected emotion images. The texture images are to be developed by researchers in order to capture specific texture attributes which can be easily understood by consumers. The combination of consumer created image boards and researcher 
developed texture cards can likely provide an effective and dynamic method for understanding consumer emotions as they related to texture and provide the opportunity for development of emotion specific products.

\subsubsection{Emotion Method Findings}

This study investigated the development of a novel emotion testing method for use with consumers and how the testing methods differ in terms of changes in emotional responses using three product categories. The words only emotion testing method had significantly greater changes between emotional responses compared to the My Pictures emotion testing when comparing the caring (Table 4.5) and self-confident (Table 4.7) emotions and greater than My Pictures and Predefined pictures when comparing the sociable emotion (Table 4.6). The standard deviation $(S D)$ was significantly greater for the words only method among a positive emotion and the $S D$ variability for My Pictures was significantly greater for a negative emotion. Additionally, the My Picture images used varied by the type of emotion, positive or negative, and could be universal or individual/context-driven images.

These findings suggest when personal self-pictures are used to identify emotions the My Pictures method is less variable than the words only method; however, the words only method appears to be less variable than the My Pictures method when non-self images are used (specifically in negative emotions). Perhaps if consumers were required to use images of themselves to portray all emotions, the My Pictures method would be less variable across all emotion types (positive, neutral, and negative) compared to the words only method. The personal pictures used in the My Pictures method may act as an 
emotional anchor for consumers, providing a contextual representation of the emotion as opposed to the emotion expressed by others (observed in Predefined pictures method and negative emotion My Picture images).

In regard to gender differences, minor differences were seen between male and female changes in emotion. Although there was a significant whole model effect between the changes in emotion and gender, only three changes in emotion had significantly different responses by gender: caring (Table 4.8), sociable (Table 4.9), and sad (Table 4.10). Despite the MANOVA whole model effect being significant, only three out of 12 significant changes in emotion occurred, which gives rise to the question as to whether there was truly a significant difference in male and females emotional responses overall. The number of male and female participants was not even, given approximately $70 \%$ women and $30 \%$ men; this could have played a substantial role in determining significant differences between genders. Additionally, considering the study was preliminary the emotions asked may not have been relevant for determining differences in emotional responses between males and females. The MANOVA results appear to concur with other findings which have suggested differences between men and women in their emotional responses (King et al., 2010; Kring \& Gordon, 1998; Seo et al., 2009; Macht, 1999; Costanzo et al., 1999); however, some limitations within this study hinder the conclusion that an obvious difference in emotional responses between males and females exist. Future research should investigate the changes in emotion between males and females with similar sample sizes and perhaps among a broader range of emotions for assessing more of the human experience with eating. 


\subsubsection{Current Emotion Methodologies}

Recently, a variety of different emotion testing methods have been developed to better understand the typical consumer's emotional response to food products. Traditionally, consumers are not provided visual aids to help elicit emotional responses during emotion testing with food (Macht, 1999; Macht \& Simons, 2000; King et al., 2010; Thomson et al., 2010). Although the use of words has many advantages in emotion testing, for example simplicity and easy of communication, its use has its disadvantages and has been criticized. Thomson et al. (2010) stated the two main criticisms of emotion words are: 1) the use of scaling may cause subjects to think about the meaning of the words, thus inducing "cognitive thought processes" while the influence of purchase intent and liking may be due to "irrational influences", and 2) the rational or cognitive thought process may cause the subject to interpret the word's literal meaning as opposed to the metaphoric meaning. Considering these disadvantages of emotion words, the use of emotion pictures may be an avenue worth pursuing for emotion testing in a food related context. It is thought that language and logic are more effectively processed in the left hemisphere of the brain, while emotions and picture images are better processed in the brain's right hemisphere (Freberg, 2009). Perhaps the use of images may limit rational or cognitive thought processes during emotion testing and yield more consistent emotional responses by encouraging the irrational processes to be more prominent.

Images have been used in a variety of different ways for emotion testing, film clips have been used to elicit negative and neutral emotions (Kring \& Gordon, 1998), while other studies have used images of food in attempt to elicit certain emotional responses or reward mechanisms in subjects (Blechert et al., 2010; Piech et al., 2010). 
Gard and Kring (2007) used 60 images to elicit emotions, equally balance across positive, neutral, and negative, amongst subjects in a non-food related context to understand sex differences and emotion; although no control to evoke emotions was used, the use of pictures appeared effective and appropriate for emotion testing. Recently, Croy et al. (2011) investigated the emotion evoking ability of olfactory stimuli versus emotion pictures and no emotion stimuli. Their results suggested the benefits of a stimulus, either olfactory or visual, to evoke emotions; additionally subjects were able to name more emotion elicitors through visual stimuli compared to olfactory stimuli for anger and sadness (Croy et al., 2011). Results of the current study indicated greater variability for no emotion stimuli (words only) in the positive emotions, and greater emotional variability in the My Pictures method in the negative emotions. Perhaps the ideal type of stimuli to evoke emotions (pictures, words, or odors) depends on the type of emotions the researcher is pursuing (positive, neutral, or negative emotions); future research should investigate this potential relationship between stimulus and emotion type and potential for product specific emotions. Regardless, the use of a stimulus (beyond words) appears to be a beneficial technique for emotion testing to aid in eliciting specific emotions.

In addition to the use of emotion images, this study captured the change in emotion frequencies across the eating experience of three product categories. Macht and Simons (2000) argue the importance of capturing emotion frequencies, suggesting greater frequencies of an emotion during eating may indicate a stronger relationship than other emotions in an eating context. Furthermore, Gard and Kring (2007) suggested the benefit of capturing the change in emotion rather than a "snapshot"; by capturing emotional responses at multiple time points provides a better understanding of the relationship 
between emotions and eating. The current study's results provided additional information about changes in emotion across the eating experience, but also the strength of that change. The use of several food product categories was paramount for observing these changes across several food forms; similar results were seen across all three product categories.

As emotion testing becomes more frequent, the investigation, development and testing of additional methods should be investigated in order to create the most effective and efficient testing methods. This study provides relevant industry information through the investigation of emotion testing with beyond basic emotions, frequencies and changes in emotions, and the use of personal and prescribed emotion images for testing. The use of personal consumer derived emotion images provides a relatively simple and reproducible method for measuring consumer emotions. Accurate and reproducible emotion data may eventually provide insight on consumer's purchase intent and the emotional power of occasion and brands. Although this study's findings are helpful for investigating alternative emotion testing methods, this work is preliminary and several limitations exist. Firstly, the questionnaire was lengthy with asking initial emotion(s), beginning, middle and end texture attributes and overall, flavor, and texture liking, and end emotions. The length of the questionnaire may have had a confounding effect on the end emotions which could alter the change in emotion results; when possible, future studies should work to limit the length of the questionnaire used for emotion testing or consider more refined objectives. Secondly, the subjects were randomly allocated a new emotion testing method for each day of testing; consumers may have experienced some "carryover" from the previous day's emotion testing method. The severity of the potential 
carryover is unknown, but may be trivial do to consumers already knowing the emotion words and their own images prior to testing.

Further research should investigate: 1) the use of self/individual emotion pictures of the subject versus predefined emotion images and words, 2) additional emotions for testing that go beyond basic emotions or are specific to certain products, and 3) alternative scaling methods which move away from the rational/word usage and towards a more non-cognitive method of capturing emotional responses from consumers.

\subsubsection{Texture Method Findings}

This study developed and tested an innovative procedure for texture measurement with consumers by exploring the relationship between imaged based testing aids and words only methods. The effectiveness of the texture methods varied when comparing them by changes in texture attributes. When considering all the data combined and orange soda only, a significant difference was observed between the two methods in the changes in texture attributes; however, no significant difference was observed for dairy beverages and cheese. It is possible when consumers were re-randomized a new testing method for the second and third day of testing there was "carryover" from the previous testing days, especially if the consumer had a texture card on the first day, but not on the following testing days. The texture cards had not been viewed prior to the first day of testing; perhaps the results of the first day are the most accurate account between the texture cards and texture words. This considered, perhaps the orange soda data (tested on the first day) should be used for comparing the two methods. Perhaps if randomization of testing methods was only done on the first day of testing there would be a clearer 
distinction between the two methods; however, based upon the data of the first day there appears to be some differences between the two methods for the change in texture attributes across the eating experience.

When exploring changes in emotion by texture method, the texture words method had consistently more significant differences between emotions compared to the texture cards method (Table 4.11). The texture words method indicated greater differences between the emotions compared to the texture cards method, suggesting the texture words method may actually provide a clearer distinction between emotions compared to the texture cards method. Perhaps the texture cards, while acting as a texture anchor, may have also decreased differences in emotions consumers might typically experience when consuming the product; by providing consumers specific texture images this may have inhibited the array of emotional experience usually experienced by the consumer during consumption. Although, when comparing each individual change in emotion by texture method, no significant differences were observed; perhaps the texture method used effects the elicitation of different emotions across the eating experience, but not necessarily effecting the changes in each emotion. Based upon the results of this study, the texture methods appeared to have little effect on the consumer's changes in emotional response during the eating experience, but may affect the differences seen between the difference emotions.

This study provides a preliminary look at emotion and texture testing with consumers through the development and implementation of two novel testing methods for emotion and texture. Texture images may be beneficial for use with consumers because the images could act as a visual anchor during testing which may decrease 
variability within and across consumers while allowing researchers to ask more specific texture questions than traditionally asked with consumers. The use of texture image cards may be additionally beneficial for testing concurrently with emotion testing as images, emotions, and oral texture are thought to be mostly processed in the orbitofrontal cortex (Rolls \& Grabenhorst, 2008; Freberg, 2009). Perhaps the development of methods which utilize the proximity of these processes will yield consistent emotion and texture attribute testing among consumers.

\subsubsection{Current Texture Measurement Methodologies}

Instrumental texture analysis assessments are often related to descriptive sensory texture attributes to understand the textural quality of foods (Breene, 1975); however, little is known about consumer reactions to texture and avenues for modifying physical characteristics to maximize consumer acceptability (Moskowitz, 1987). Foegeding et al. (2011) states, "human perception of any product is best and optimally measured by humans, not machines," perhaps then consumer related texture questions should be answered by consumers, providing the opportunity for consumer focused alternative texture methods. Consumers are typically asked acceptance and liking questions about texture, but perhaps through the use of texture image cards, developed by sensory scientists and product developers, a clearer understanding of how consumers perceive textural changes in food and how these specific textural attributes are related to product liking and acceptability could be obtained. Little information is known about the effectiveness of texture image cards with consumers especially in the context of emotions, but one study investigated the effect of texture on the relationship between certain colors and emotions (Lucassen et al., 2011). The results suggested the addition of 
visual textures played a significant role in the color/emotion relationship on a hard-soft scale, and a non-significant trend for masculine-feminine, heavy-light, and warm-cool emotion scales (Lucassen et al., 2011). Although Lucassen et al. (2011) did not investigate oral texture, it does suggest a potential relationship between texture and emotions, and further investigation of this relationship appears to be justified. Our study provides a preliminary look at the use of texture images cards for use with consumers and a glimpse of texture and emotion testing by way of alternative testing methods.

Instrumental texture measurements have been around for decades; however, these measurements are often correlated to descriptive sensory texture assessments rather than consumer perceptions of food textures. Instrumental and descriptive sensory texture measurements are essential for a variety of different objectives within the food industry; however, for consumer texture research, alternative methods may be a route worth pursuing. Utilization of food texture cards for use by consumers may provide important consumer texture perception data without the use of instrumental or descriptive sensory testing, thus saving valuable time, money, and resources. The use of texture image cards for other food forms should be investigated to better understand the effectiveness of these cards over a wide variety of textures. Additionally, researchers should continue to explore the effect of specific textural attributes on certain emotions. Consumer texture attribute responses could be compared to TPA or other instrumental texture measurements to understand the relationship between consumer-perceived texture and instrumental texture assessments. 


\subsection{Conclusion}

The use of emotion testing has greatly increased within sensory science; however, alternative methods should be pursued to create an ideal emotion testing method. Due to the non-cognitive nature of emotions, they can be difficult to capture and quantify therefore methods should be created to measure emotions in the most natural way possible. Images and emotions are thought to be processed in the same cerebral hemisphere and through similar pathways, thus a visual emotional stimulus maybe key to accurately capturing emotions. The current study's results suggest the images used (emotion images of self, images of people the subject knows but not self, or pictures of others/strangers) for testing may be the key to the consistency and reproducibility of using emotion images rather than words only. The use of personal emotion images appears to be a promising emotion testing method and should be investigated further.

A variety of different instrumental texture analysis methods have been developed and are frequently used to correlated instrumental texture measurements to descriptive sensory analysis; perhaps specific texture attributes can be tested with consumers if the right texture method is used. For samples tested on the first day (clearest distinction between the two methods) there was a significant difference between the changes in textures by texture method. The use of texture image cards provides an opportunity to capture consumer perceived texture attributes rather than instrument or descriptive sensory panel texture analysis. Texture images appear to be an appropriate method for capturing specific texture attributes and exploration of different emotional responses with consumers. This first look at the IMET methodology demonstrated the advantages and disadvantages of assessing emotion and texture simultaneously among consumers. 


\title{
$\underline{5.0}$
}

\section{Evaluating Consumer Emotional Responses and Liking to Non-Commercial Low Sodium Mozzarella Cheese}

\begin{abstract}
Americans, on average, consume far beyond the recommended daily allowance of sodium, thus putting them a higher risk of hypertension, cardiovascular disease, and stroke. Mozzarella cheese is currently the most popularly consumed cheese in the U.S. and provides approximately $8 \%$ DV of sodium in one string cheese stick (approximately 28g); thus mozzarella cheese appears to be an appropriate food for researching sodium reduction. Sodium reduction has been challenging for manufacturers considering consumers view low sodium products as bland and flavorless.
\end{abstract}

The current study employed the use of traditional acceptability testing in addition to a novel method for evaluating subject's emotional responses to products in order to obtain additional information above and beyond what traditional sensory testing methods provide to researchers. Eight cheese variables were tested, seven low sodium levels and one full sodium sample, in order to determine which salt replacer was the most liked by subjects and how subject's emotions varied between samples. Instrumental texture analysis was also performed to ensure the textural quality of the low sodium cheeses was maintained and comparable to the texture attributes of the full sodium cheese.

The study's results indicated: 1) the full sodium cheese was consistently the most preferred sample for all liking attributes, followed by the $100 \% \mathrm{KCl}$ cheese variable, 2) low sodium variables including $50 \%$ Salona, $100 \% \mathrm{KCl}$, and $50 \% \mathrm{KCl}$ samples were able 
to elicit similar emotional responses to those experienced during consumption of the full sodium cheese, and 3) higher mean scores for the tested texture attributes were associated with the most liked cheese samples. The $100 \% \mathrm{KCl}$ low sodium cheese variable was recommended for further research in low sodium cheese testing.

Highlights: >Production of eight variables of bacteria cultured mozzarella cheeses were carried out with seven variables of low sodium cheese with different salt replacers. >Acceptability testing and emotional responses were captured for all eight samples across two days of testing to determine changes in liking and emotions. >Texture analysis was performed on all cheese samples to ensure textural quality and comparison of texture measurements between the selected low sodium sample and the full sodium cheese.

Keywords: emotion testing, hedonic testing, texture analysis, non-commercial low sodium mozzarella cheese 


\subsection{Introduction}

Hypertension is a leading risk factor for the two most frequent causes of death, cardiovascular disease and stroke, in the United States, and hypertension is associated with increased sodium consumption. The alarmingly high rates of hypertension among the American population has given rise to a number of initiatives for decreasing sodium content in a variety of food products (CDC, 2012; NHLBI, 2012; USDA, 2010). Given mozzarella cheese is currently the most consumed cheese in the United States, it's a clear avenue for pursuing sodium reduction (IDFA, 2012). Sodium reduction in a variety of different cheeses including: cheddar, feta, and imitation cheeses (Grummer et al., 2012; Katsiari et al., 1997; El-Bakry et al., 2011), has been somewhat successful; however, the traditionally mild/bland flavor of mozzarella cheese offers a major challenge for decreasing salt content while still delivering the same flavor, saltiness, and texture consumers expect. Additionally, consumer researchers are pursuing alternative methods for measuring the consumer's experience with food, and capturing the consumer's emotional response to products appears to be a method worth investigating (King \& Meisleman, 2010; King et al., 2010; Macht, 1999; Macht, 2008; Macht \& Simons, 2000). Perhaps the limited success of low sodium products is due to researchers asking consumers the wrong questions about what really drives them to purchase, consume, and enjoy products; through emotion measurement, a better understanding of the consumer's mindset may be obtained.

The current study manufactured seven varieties of low sodium bacteria cultured mozzarella cheese and one variable of full sodium cheese. These eight samples were used for traditional acceptability testing and for capturing the subject's change in emotional 
responses to the products, using a unique method of emotion measurement (IMET), across two days of testing. Lastly, instrumental texture analysis was performed on the samples to determine the effects of lowering sodium on certain texture attributes of the cheese. The objectives of this study were to: 1) identify one salt replacer which was consistently most preferred compared to the other variables and ideally not significantly different in liking compared to the full sodium control, 2) determine if similar emotional responses could be elicited in a low sodium cheese as those evoked when consuming the full sodium sample, and 3) identify which texture attributes were associated with the samples which were most liked.

Products with higher salt content were hypothesized to have higher subject acceptability scores and would have increased changes in positive emotions, while the low sodium cheeses were hypothesized to be liked less by subjects and have greater increases in negative emotions; these hypotheses are based upon previous results from the researchers, Development of a Novel Method for Evaluating Emotions and Texture: Imagery and Language (Collinsworth et al., 2013). Additionally, significant differences in changes between the first and last emotional responses and first and last liking scores were expected, and liking scores were thought to decrease between the first and second day of testing. The full sodium cheese sample was hypothesized to have significantly different texture attribute measurements compared to the low sodium cheeses, and the more liked samples would have significantly different texture attribute measurements compared to the disliked samples. 


\subsection{Methodology}

\subsubsection{Materials}

Unsalted bacteria cultured mozzarella cheese curd manufactured by Rizo Lopez Foods, Inc. (Modesto, CA) was used for testing with eight cheese variables; curd was kept refrigerated $\left(37^{\circ} \mathrm{F}\right)$ prior to use. Curd was weighed then placed into a cookerstretcher (Blentech, model CC-45) and the curd was made molten $\left(160-170^{\circ} \mathrm{F}\right)$ by low auger agitation and heat. Predetermined salt and salt replacer amounts were added to the molten curd in the cooker-stretcher and were allowed approximately five minutes to combine. After mixing, each cheese variable was placed into a metal rectangular cheese hoop lined with cheese cloth, then placed in refrigeration $\left(37^{\circ} \mathrm{F}\right)$. Salt replacers used for testing included: $\mathrm{NaCl} / \mathrm{KCl}$ blend ( $\mathrm{KCl}$ by Morton Salt, lot number: APR102HK01), ALTA $^{\text {TM }} 2345$ (Kerry, ID number: s-341825), and Salona (BK Giulini, lot number: 15APR2011-F). The antimicrobial dip used for testing was SEA-i F75 (Bienca, lot number: 101027 ) at $0.275 \%$.

\subsubsection{Samples}

A total of eight variables were used for testing, the eight samples included: full sodium, low sodium, $100 \% \mathrm{NaCl} / \mathrm{KCl}$ blend, $50 \% \mathrm{NaCl} / \mathrm{KCl}$ blend, $100 \%$ ALTA $^{\mathrm{TM}}, 50 \%$ ALTA $^{\mathrm{TM}}$, 100\% Salona, and 50\% Salona (Table 5.1). 
Table 5.1. Specifications for Salt Replacer Cheese Variables and Controls. Eight variables were made for testing with consumers; blends of $\mathrm{NaCl}$ and a salt replacer were calculated to fulfill the maximum limit of sodium allowed in a low sodium cheese.

\begin{tabular}{|c|c|c|c|c|c|}
\hline \multicolumn{6}{|c|}{ Specifications for Salt Replacer Cheese Variables and Controls } \\
\hline $\begin{array}{c}\text { Numple } \\
\text { Sumber }\end{array}$ & $\begin{array}{c}\text { Amount of NaCl } \\
\text { Name }\end{array}$ & $\begin{array}{c}\text { Salt } \\
\text { per 20lbs cheese } \\
\text { block) }\end{array}$ & $\begin{array}{c}\text { Amount of Salt } \\
\text { Replacer (per } \\
\text { 20lbs cheese } \\
\text { block) }\end{array}$ & $\begin{array}{c}\text { Dipped in SEA-i } \\
\text { F75 @ 0.275\% }\end{array}$ \\
\hline 1 & Full Sodium & $250 \mathrm{~g}$ & None & N/A & Yes \\
\hline 2 & Low Sodium & $117.5 \mathrm{~g}$ & None & N/A & Yes \\
\hline 3 & $100 \% \mathrm{KCl}$ & $100 \mathrm{~g}$ & $\mathrm{KCl}$ & $81 \mathrm{~g}$ & Yes \\
\hline 4 & $50 \% \mathrm{KCl}$ & $100 \mathrm{~g}$ & $\mathrm{KCl}$ & $40.5 \mathrm{~g}$ & Yes \\
\hline 5 & $100 \%$ ALTA & $75 \mathrm{~g}$ & ALTA 2345 & $20.4 \mathrm{~g}$ & Yes \\
\hline 6 & $50 \%$ ALTA & $75 \mathrm{~g}$ & ALTA 2345 & $10.2 \mathrm{~g}$ & Yes \\
\hline 7 & $100 \%$ Salona & $90 \mathrm{~g}$ & Salona & $77.6 \mathrm{~g}$ & Yes \\
\hline 8 & $50 \%$ Salona & $90 \mathrm{~g}$ & Salona & $38.8 \mathrm{~g}$ & Yes \\
\hline
\end{tabular}

The salt content of the full and low sodium samples was analyzed using the Corning Ion Analysis Method No. 10 and a Corning Chloride Analyzer 926 and the cheese moisture content was measured using CEM LabWave 9000 Microwave Moisture/Solids Analyzer (model No: 910800); the salt and moisture content of cheese samples were analyzed by DPTC staff.

Cheese samples used for the first day of testing were 31 days post addition of salt replacers, and cheese samples for the second day of testing were 51 days post addition of salt replacers. All samples were cubed to $3 / 4$ inches and dipped in a $0.275 \%$ SEA-i F75 solution for two minutes then allowed to air dry for 30-45 minutes. Sample were then placed in two ounce clear plastic SOLO® soufflé cups (SOLO® Cup Company, Lake Forest, IL) with randomized three-digit codes and cupped. Cheese samples were kept at $37^{\circ} \mathrm{F}$ overnight prior to testing, and samples were kept refrigerated until 30 minutes before testing, at which point samples were kept at room temperature $\left(72^{\circ} \mathrm{F}\right)$ and were 
served to subjects at this adjusted temperature. Samples were presented in a William's design, where sample order was randomized within and across subjects.

\subsubsection{Subjects}

Participants for this study were recruited by email and flyers; subjects were selected for testing based upon their willingness to consume low sodium string cheese, frequently consuming mozzarella cheese, consuming a nutritional alternative dairy product (including low fat cheese, ice cream, milk or low/reduced sodium cheese), and being a non-user of Crest-Pro health products. Once selected for testing, subjects were asked to complete "homework" prior to arriving to the testing site. For "homework", participants were given a poster board with 12 sections, each with a unique emotion word with three to four associated adjectives; subjects were required to attach one image which they found best represented each of the 12 emotion words. A total of 85 subjects $(20.5 \pm$ 2.5 years), including students, university staff, and community members participated in the first day of testing with 65 females (76.5\%) and 20 males (23.5) and 61 subjects participated in the second day of testing with 48 females (79\%) and 13 males (21\%). Subjects received a total of thirty-five dollars in gift cards for participating in the two days of testing, with twenty-five dollars for the first day and ten dollars for the second day.

\subsubsection{Questionnaire and testing procedure}

The questionnaire involved asking the subject their initial emotional state in a choose-all-that-apply (CATA) format prior to consuming the sample, the twelve emotions consisted of: caring, excited, sociable, self-confident, surprised, detached, 
fatigued, inferior, judgmental, raging, sad, and fear, a "none of these" option was also included. Emotions selected for testing were selected based upon previous research (Thomson \& Crocker, 2011) where hundreds of emotions were testing with consumers across four countries to determine a more refined list of emotion testing words. All subjects were instructed to use the images on their personal poster board to help elicit or anchor the subject to the emotions listed. Subjects were then directed to consume and score the hedonics of a given product, liking attributes included: overall flavor, saltiness, texture, appearance, and aroma liking. Subjects were asked to score each hedonic attribute when first consuming, halfway through consuming, and at the end of consuming the product. Upon completing the hedonic portion, subjects entered their emotional state after consuming the product, in the same format as presented for assessing the initial emotions. Consumers were asked to comment on what aspect of the product they liked the most before moving onto the next sample. A ten second delay occurred between each sample where subjects were asked to rinse their palate with the provided drinking water and unsalted crackers to help decrease carryover and sensory fatigue. Testing was performed using Compusense® at-hand.

On average, subjects were able to complete the test in 20 minutes. Testing was conducted in an open lab, where subjects were not in sensory booths but rather dispersed around a "U" shaped table; subjects were monitored to reduce noise or conversations between subjects. Sensory testing was implemented at California Polytechnic State University - San Luis Obispo, and was performed twice with the same subjects with the second day of testing occurring 20 days after the first day of testing. Subject selection and 
sensory testing procedures were reviewed and approved by the California Polytechnic State University - San Luis Obispo Institutional Review Board prior to testing.

\subsubsection{Texture Analysis}

Upon completion of all sensory testing, instrumental texture analysis of all eight cheese variables was performed using the TA-XT2 Texture Analyzer (Texture Technology Corporation, Scarsdale, NY). Cheese samples were cut into two centimeter cubes, and covered and refrigerated for four hours prior to testing. Cheese samples were left out at room temperature $\left(72^{\circ} \mathrm{F}\right)$ for 30 minutes prior to analysis and sample analysis order was randomized. The texture profile analysis (TPA) method was used for testing the eight cheese variables, and each cheese variable was tested five times. The TPA method traditionally measures the following attributes: fracturability, hardness, cohesiveness, adhesiveness, springiness, gumminess, and chewiness (Bourne, 1978); data for measuring all TPA attributes were captured, expect for fracturability and adhesiveness. Pons and Fiszman (1996) state the definitions for each of the five tested TPA attributes as defined previously by Bourne (1978) as:

- Hardness is "the peak force during the first compression cycle" ("first bite").

- Cohesiveness is "the ratio of the positive force area during the second compression portion to that during the first compression..."

- Springiness is "the height that the food recovers during the time that elapses between the end of the first bite and the start of the second bite."

- Gumminess is "hardness X cohesiveness."

- Chewiness is "gumminess X springiness." 
Table 5.2 contains the testing parameters for the texture analysis.

Table 5.2. Texture Analysis Settings. Specific settings selected for testing eight non-commercial cheese samples with the TA-XT2 Texture Analyzer.

\begin{tabular}{|c|c|}
\hline \multicolumn{2}{|c|}{ Texture Analysis Settings } \\
\hline Settings & Selected Settings \\
\hline Test Mode & T.P.A \\
\hline Pre Test Speed & $1.2 \mathrm{~mm} / \mathrm{s}$ \\
\hline Test Speed & $1.2 \mathrm{~mm} / \mathrm{s}$ \\
\hline Post Test Speed & $1.2 \mathrm{~mm} / \mathrm{s}$ \\
\hline Distance & $10 \mathrm{~mm}$ \\
\hline Compression & $50 \%$ \\
\hline Time & $5.00 \mathrm{~s}$ \\
\hline Force & $5 \mathrm{~g}$ \\
\hline Probe Style & $1.5^{\prime \prime}$ polymer cylinder probe \\
\hline
\end{tabular}

\subsection{Data Analysis}

All statistical analysis was performed using JMP (JMP, Version 10. SAS Institute Inc., Cary, NC).

\subsubsection{Product Hedonic Analysis}

The statistical analysis for comparing the hedonic scores by each cheese variable was carried out using an oneway ANOVA. Three instances of liking (first, middle, end) for each liking attribute were captured, for this analysis the three instances were averaged to obtain a mean for each of the five hedonic attributes. Although the hedonic scores were captured categorically via the 9-point scale, they were treated at continuous data in order to compare the means and variance across each variable. If a hedonic test p-value was significant ( $\alpha=0.05)$, a Tukey's HSD (5\%) post hoc comparison was performed to identify where the specific differences existed within the comparison. Normality and homogeneity of variances assumptions were checked prior to data analysis, the Kruskal- 
Wallis ranked sum test was used for analysis of data when the normality assumption was not met and Welch's test was used for data which did not meet the homogeneity of variances assumption ( $\mathrm{p}$-value $<0.01)$.

\subsubsection{Comparison of First, Middle and End Liking Scores}

In order to understand how the liking scores changed across the eating experience, an oneway ANOVA was employed to compare the change in each hedonic attribute (overall flavor, saltiness, texture, appearance, and aroma) between the first, middle, and last hedonic scores; the first, middle, and last hedonic scores were compared within each of the five different hedonic attributes. A global p-value of less than 0.05 justified a Tukey's HSD post hoc comparison for identifying which hedonic time points were significantly different (5\%). Normality and homogeneity of variance assumptions were checked, and the Kruskal-Wallis ranked sum test for concerns with normality and Welch's test for issues with unequal variances were used when necessary.

\subsubsection{Liking Score Change between Day 1 and Day 2}

For assessing the changes in liking scores between the two days of testing, the average of the three time points for each hedonic attribute were calculated for both days. The average for each hedonic attribute from day one was compared to the average of the same hedonic attributes in day two by way of a two factor analysis of variance comparing each average hedonic attribute by day and cheese variable; possible interactions between day and cheese variable were also explored. P-values less than 0.05 were considered significantly different and thus a significant change in the average hedonic score of the given hedonic attribute occurred between the two days. Normality and homogeneity of 
variances assumptions were checked, and where appropriate the Wilcoxon signed ranked test and Welch's test p-values and test statistics were utilized.

\subsubsection{Comparison of Each Change in Emotion by Cheese Variable}

To analyze the change in emotion, the first emotion score was subtracted from the end emotion score for each of the emotions; an oneway ANOVA was used for comparing the change in each emotion across the eight cheese variables. Although the emotion scores were captured as categorical data, they were analyzed as continuous to evaluate the direction of the emotion change and that relationship to the type of emotion (positive, negative, or neutral) being assessed and to the cheese product. Considering the issues which may occur when analyzing categorical data as continuous, the Kruskal-Wallis rank sum test was used for all emotion data to help alleviate concerns with the lack of normality in the data. Given the lack of normality in this data, the Brown-Forsythe pvalue was used for assessing equality of variances due to its decreased sensitivity to nonnormal data compared to the Levene's test, p-values less than 0.01 were considered unequal. Tests with Kruskal-Wallis p-values less than 0.05 were considered significant and the Steel-Dwass all pairs nonparametric post hoc comparison at $(\alpha=0.05)$ was used for determining which specific cheese samples differed by change in each emotion. Note: the Steel-Dwass all pairs post hoc comparison is the nonparametric equivalent to the Tukey's HSD all pairs comparison, and takes into consideration the family-wise error rate (JMP, 2012). 


\subsubsection{Comparison of First Emotion Scores to End Emotion Scores}

For both days of testing, the first emotional responses for each emotion were compared to the end emotional responses by way of a matched pairs analysis, considering the lack of independence between the two groups. The emotion data was analyzed as continuous data, and to help with issues of non-normality the Wilcoxon signed rank test was used for analysis. Comparisons with p-values less than 0.05 were considered significantly different, and thus suggesting a significant change in a given emotion had occurred across the eating experience. The Brown-Forsythe p-value was used for assessing equality of variances between the groups ( $\mathrm{p}$-value $<0.01)$.

\subsubsection{Emotional Changes between Day 1 and Day 2}

In order to assess if any significant changes in emotion occurred between the two days of testing, the change in emotion (end - first) for each emotion from the first day was compared to the changes in emotion from the second day by way of a matched pairs analysis. The Wilcoxon signed rank test was used with a significance level of $\alpha=0.05$, thus emotion comparisons with p-values less than this criteria were considered significantly different and suggested a significant change in emotion between the two days of testing. The Brown-Forsythe ( $\mathrm{p}$-values < 0.01) was used for assessing homogeneity of variances.

\subsubsection{Comparison of Change in Emotion by Gender}

All emotional changes (end - first) were compared between men and women, and for both days of testing. A MANOVA was performed comparing the change in all emotions by gender for both days; given a significant global p-value, additional 
investigation to determine which emotions differed significantly by gender was implemented. Each change in emotion was considered continuous, and the Wilcoxon rank test was used for the ANOVAs at $\alpha=0.05$; change in emotion by gender comparisons with p-values less than 0.05 were considered significantly different and thus one gender had different changes in an emotion compared to the other gender. The Brown-Forsythe (p-values $<0.01$ ) was used for assessing homogeneity of variances.

\subsubsection{Texture Analysis}

Each of the five TPA attributes: hardness, cohesiveness, springiness, gumminess, and chewiness, were compared across the eight cheese variables in an oneway ANOVA; homogeneity of variances and normality assumptions were checked prior to interpretation of results. The Welch's test was used for interpreting results of tests which did not meet the homogeneity of variances assumption, and the Kruskal-Wallis test was used for tests which did not meet the normality assumption. P-values less than 0.05 were considered significantly different and the Steel-Dwass all-pairs post hoc comparison was performed to determine which samples were significantly different from one another $(\alpha=0.05)$. If the data met the normality assumption, Tukey's HSD method was used for post hoc comparisons.

\subsection{Results}

\subsubsection{Product Hedonic Analysis}

When comparing the average liking of the five hedonic attributes by the eight cheese variables for the first day of testing, a significant difference was determined in overall flavor liking $\left(\mathrm{F}_{7,286.88}=54.4, \mathrm{P}<0.0001\right)$, saltiness liking $\left(\mathrm{F}_{7,672}=27.4, \mathrm{P}<\right.$ 
0.0001), texture liking $\left(\mathrm{F}_{7,672}=23.5, \mathrm{P}<0.0001\right)$, appearance liking $\left(\mathrm{Chi}-\mathrm{Sq}{ }_{7}=35.9, \mathrm{P}<\right.$ 0.0001), and aroma liking $\left(\mathrm{Chi}-\mathrm{Sq}_{7}=57.3, \mathrm{P}<0.0001\right)$. Due to issues with homogeneity of variances, Welch's test was used for overall flavor liking and due to issues with normality the Kruskal-Wallis test was used for appearance liking and aroma liking. The post hoc comparison for the first day can be seen in Table 5.3 for all significant attributes: overall flavor liking, saltiness liking, texture liking, appearance liking, and aroma liking; variables which do not share difference letters are significantly different $(5 \%)$.

For the second day of testing, significant differences were observed for overall flavor liking $\left(\mathrm{F}_{7,480}=28.5, \mathrm{P}<0.0001\right)$, saltiness liking $\left(\mathrm{F}_{7,480}=20.5, \mathrm{P}<0.0001\right)$, texture liking $\left(\mathrm{F}_{7,480}=18.2, \mathrm{P}<0.0001\right)$, appearance liking $\left(\mathrm{Chi}-\mathrm{Sq}_{7}=50.5, \mathrm{P}<0.0001\right)$, and aroma liking $\left(\mathrm{Chi}-\mathrm{Sq}_{7}=55.8, \mathrm{P}<0.0001\right)$. Due to issues with normality the KruskalWallis test was used for appearance liking and aroma liking. The post hoc comparison for the second day can be seen in Table 5.4 for all significant hedonic attributes; variables which do not share difference letters are significantly different (5\%). 
Table 5.3. Hedonic Attribute Post Hoc Comparison of Day 1. Post hoc comparison of day 1 (31 days post salt addition) for all significant hedonic attributes.

\begin{tabular}{|c|c|c|c|c|c|c|c|c|c|}
\hline \multicolumn{10}{|c|}{ Hedonic Attribute Post Hoc Comparison of Day 1 (31 Days Post Addition of Salt Replacers) } \\
\hline \multicolumn{2}{|c|}{ Overall Flavor Liking } & \multicolumn{2}{|c|}{ Saltiness Liking } & \multicolumn{2}{|c|}{ Texture Liking } & \multicolumn{2}{|c|}{ Appearance Liking } & \multicolumn{2}{|c|}{ Aroma Liking } \\
\hline Variable & Mean \pm SD & Variable & Mean \pm SD & Variable & Mean \pm SD & Variable & Mean \pm SD & Variable & Mean \pm SD \\
\hline Full Sodium & $7.40 \pm 1.07 \mathrm{a}$ & Full Sodium & $7.05 \pm 1.30 \mathrm{a}$ & Full Sodium & $6.97 \pm 1.52 \mathrm{a}$ & Full Sodium & $6.53 \pm 1.29 \mathrm{a}$ & Full Sodium & $6.8 \pm 1.25 \mathrm{a}$ \\
\hline $100 \% \mathrm{KCl}$ & $5.87 \pm 1.68 b$ & $100 \% \mathrm{KCl}$ & $5.50 \pm 1.68 \mathrm{~b}$ & $100 \% \mathrm{KCl}$ & $5.96 \pm 1.71 b$ & $100 \% \mathrm{KCl}$ & $6.11 \pm 1.38 \mathrm{ab}$ & $100 \% \mathrm{KCl}$ & $6.28 \pm 1.25 \mathrm{ab}$ \\
\hline $50 \% \mathrm{KCl}$ & $5.52 \pm 1.78 \mathrm{~b}$ & $50 \% \mathrm{KCl}$ & $5.32 \pm 1.52 \mathrm{bc}$ & $100 \%$ Salona & $5.72 \pm 1.72 \mathrm{~b}$ & $50 \%$ Salona & $6.10 \pm 1.19 \mathrm{ab}$ & $50 \% \mathrm{KCl}$ & $6.13 \pm 1.29 \mathrm{bc}$ \\
\hline $50 \%$ Salona & $5.49 \pm 1.56 \mathrm{~b}$ & $50 \%$ Salona & $5.24 \pm 1.54 \mathrm{bc}$ & $50 \% \mathrm{KCl}$ & $5.69 \pm 1.71 \mathrm{~b}$ & $50 \% \mathrm{KCl}$ & $6.07 \pm 1.35 \mathrm{ab}$ & $100 \%$ Salona & $5.97 \pm 1.22 \mathrm{bcd}$ \\
\hline $100 \%$ Salona & $5.27 \pm 1.74 \mathrm{~b}$ & $100 \%$ Salona & $5.19 \pm 1.57 \mathrm{bc}$ & $50 \%$ Salona & $5.58 \pm 1.78 b$ & $100 \%$ Salona & $5.97 \pm 1.32 \mathrm{abc}$ & $50 \%$ Salona & $5.89 \pm 1.09 \mathrm{bcd}$ \\
\hline $100 \%$ Alta & $4.48 \pm 1.74 \mathrm{c}$ & $100 \%$ Alta & $4.66 \pm 1.65 \mathrm{~cd}$ & $100 \%$ Alta & $4.58 \pm 1.83 \mathrm{c}$ & $100 \%$ Alta & $5.80 \pm 1.34 \mathrm{bc}$ & $100 \%$ Alta & $5.58 \pm 1.33 \mathrm{~cd}$ \\
\hline $50 \%$ Alta & $4.19 \pm 1.77 \mathrm{c}$ & $50 \%$ Alta & $4.36 \pm 1.72 \mathrm{~d}$ & $50 \%$ Alta & $4.43 \pm 1.96 \mathrm{c}$ & $50 \%$ Alta & $5.64 \pm 1.39 \mathrm{bc}$ & $50 \%$ Alta & $5.5 \pm 1.52 \mathrm{~d}$ \\
\hline Low Sodium & $4.12 \pm 1.81 \mathrm{c}$ & Low Sodium & $4.09 \pm 1.73 \mathrm{~d}$ & Low Sodium & $4.20 \pm 1.83 \mathrm{c}$ & Low Sodium & $5.37 \pm 1.59 \mathrm{c}$ & Low Sodium & $5.48 \pm 1.55 \mathrm{~d}$ \\
\hline
\end{tabular}

Table 5.4. Hedonic Attribute Post Hoc Comparison of Day 2. Post hoc comparison of day 2 (51 days post salt addition) for all significant hedonic attributes.

\begin{tabular}{|c|c|c|c|c|c|c|c|c|c|}
\hline \multicolumn{10}{|c|}{ Hedonic Attribute Post Hoc Comparison of Day 2 (51 Days Post Addition of Salt Replacers) } \\
\hline \multicolumn{2}{|c|}{ Overall Flavor Liking } & \multicolumn{2}{|c|}{ Saltiness Liking } & \multicolumn{2}{|c|}{ Texture Liking } & \multicolumn{2}{|c|}{ Appearance Liking } & \multicolumn{2}{|c|}{ Aroma Liking } \\
\hline Variable & Mean \pm SD & Variable & Mean \pm SD & Variable & Mean \pm SD & Variable & Mean \pm SD & Variable & Mean \pm SD \\
\hline Full Sodium & $6.59 \pm 1.33 \mathrm{a}$ & Full Sodium & $6.21 \pm 1.53 \mathrm{a}$ & Full Sodium & $6.44 \pm 1.66 \mathrm{a}$ & Full Sodium & $6.21 \pm 1.45 \mathrm{a}$ & Full Sodium & $6.09 \pm 1.38 \mathrm{a}$ \\
\hline $100 \% \mathrm{KCl}$ & $5.61 \pm 1.88 \mathrm{~b}$ & $100 \% \mathrm{KCl}$ & $5.63 \pm 1.82 \mathrm{ab}$ & $100 \% \mathrm{KCl}$ & $5.96 \pm 1.86 \mathrm{ab}$ & $100 \% \mathrm{KCl}$ & $6.00 \pm 1.40 \mathrm{a}$ & $100 \% \mathrm{KCl}$ & $5.92 \pm 1.27 \mathrm{a}$ \\
\hline $100 \%$ Salona & $5.29 \pm 1.76 \mathrm{~b}$ & $100 \%$ Salona & $5.26 \pm 1.51 \mathrm{~b}$ & $100 \%$ Salona & $5.63 \pm 1.92 \mathrm{ab}$ & $100 \%$ Salona & $5.95 \pm 1.40 \mathrm{a}$ & $100 \%$ Salona & $5.86 \pm 1.38 \mathrm{ab}$ \\
\hline $50 \%$ Salona & $5.18 \pm 1.77 \mathrm{~b}$ & $50 \%$ Salona & $5.08 \pm 1.47 \mathrm{~b}$ & $50 \%$ Salona & $5.55 \pm 1.76 \mathrm{ab}$ & $50 \%$ Salona & $5.84 \pm 1.29 \mathrm{ab}$ & $50 \% \mathrm{KCl}$ & $5.78 \pm 1.23 \mathrm{ab}$ \\
\hline $50 \% \mathrm{KCl}$ & $4.96 \pm 1.82 \mathrm{~b}$ & $50 \% \mathrm{KCl}$ & $5.06 \pm 1.67 \mathrm{~b}$ & $50 \% \mathrm{KCl}$ & $5.33 \pm 1.78 \mathrm{~b}$ & $50 \% \mathrm{KCl}$ & $5.73 \pm 1.41 \mathrm{ab}$ & $50 \%$ Salona & $5.75 \pm 1.22 \mathrm{ab}$ \\
\hline $50 \%$ Alta & $3.77 \pm 1.65 \mathrm{c}$ & $50 \%$ Alta & $4.13 \pm 1.57 \mathrm{c}$ & $50 \%$ Alta & $4.23 \pm 1.80 \mathrm{c}$ & $50 \%$ Alta & $5.06 \pm 1.47 \mathrm{bc}$ & $50 \%$ Alta & $5.15 \pm 1.32 \mathrm{bc}$ \\
\hline $100 \%$ Alta & $3.61 \pm 2.02 \mathrm{c}$ & $100 \%$ Alta & $4.00 \pm 1.74 \mathrm{c}$ & $100 \%$ Alta & $4.01 \pm 2.06 \mathrm{c}$ & $100 \%$ Alta & $4.92 \pm 1.60 \mathrm{c}$ & Low $\mathrm{Na}$ & $4.86 \pm 1.20 \mathrm{c}$ \\
\hline Low Sodium & $3.03 \pm 1.56 \mathrm{c}$ & Low Sodium & $3.40 \pm 1.48 \mathrm{c}$ & Low Sodium & $3.67 \pm 1.83 \mathrm{c}$ & Low Sodium & $4.76 \pm 1.47 \mathrm{c}$ & $100 \%$ Alta & $4.57 \pm 1.69 \mathrm{c}$ \\
\hline
\end{tabular}




\subsubsection{Comparison of First, Middle and End Liking Scores}

Assessing the change in liking across the eating experience for the first day of testing was analyzed and significant differences were observed in overall flavor liking $\left(\mathrm{F}_{2,1350}=4.79, \mathrm{P}=0.0085\right)$, saltiness liking $\left(\mathrm{F}_{2,2037}=3.42, \mathrm{P}=0.0327\right)$, texture liking $\left(\mathrm{F}_{2}\right.$, $1355=6.70, \mathrm{P}=0.0013)$, and aroma liking $\left(\mathrm{F}_{2,2037}=5.53, \mathrm{P}=0.0040\right)$. Welch's test was used for determining significant differences in overall flavor liking and texture liking due to issues with homogeneity of variances. Generally, the first and last liking scores were significantly different, while first and middle liking scores were occasionally significantly different. Table 5.5 contains the post hoc comparisons for overall flavor liking, and saltiness liking, texture liking, and aroma liking post hoc comparisons can be observed in Table 5.6, 5.7, and 5.8 respectively; time points which do not share differences letters are significantly different (5\%).

Table 5.5. Change in Overall Flavor Liking Day 1. Table 5.6. Change in Saltiness Liking Day 1. Post hoc comparison of overall flavor liking for $\quad$ Post hoc comparison of saltiness liking for day 1. day 1.

\begin{tabular}{|c|c|c|c||c|c|c|c|}
\hline \multicolumn{4}{|c||}{$\begin{array}{c}\text { Change in Overall Flavor Liking } \\
\text { Day 1 }\end{array}$} & \multicolumn{4}{c|}{$\begin{array}{c}\text { Change in Saltiness Liking } \\
\text { Day }\end{array}$} \\
\hline Time Point & Mean & SD & \multicolumn{1}{|c|}{ Differences } & Time Point & Mean & SD & Differences \\
\hline First & 5.47 & 1.87 & A & First & 5.31 & 1.79 & A \\
\hline Middle & 5.28 & 2.07 & A B & Middle & 5.18 & 1.91 & A B \\
\hline End & 5.12 & 2.24 & B & End & 5.04 & 2.01 & B \\
\hline
\end{tabular}

Table 5.7. Change in Texture Liking Day 1. Post hoc comparison of texture liking for day 1.
Table 5.8. Change in Aroma Liking Day 1. Post hoc comparison of aroma liking for day 1 .

\begin{tabular}{|c|c|c|c||c|c|c|c|}
\hline \multicolumn{4}{|c||}{$\begin{array}{c}\text { Change in Texture Liking } \\
\text { Day 1 }\end{array}$} & \multicolumn{4}{c|}{ Day 1 } \\
\hline Time Point & Mean & SD & Differences & Time Point & Mean & SD & Differences \\
\hline First & 5.61 & 1.93 & A & First & 6.10 & 1.44 & A \\
\hline Middle & 5.35 & 2.07 & B & Middle & 5.90 & 1.46 & B \\
\hline End & 5.21 & 2.16 & B & End & 5.86 & 1.49 & B \\
\hline
\end{tabular}


For the second day of testing, liking scores across the eating experience were analyzed and no significant differences $(\alpha=0.05)$ between first, middle, and end liking were observed in any of the five hedonic attributes.

\subsubsection{Liking Score Change between Day 1 and Day 2}

A two factor (day and cheese variable) analysis of variance of average overall flavor liking revealed main effects for day $\left(\mathrm{F}_{1,1344}=16.6, \mathrm{P}<0.0001\right)$, and cheese variable $\left(\mathrm{F}_{7,1344}=75.6, \mathrm{P}<0.0001\right)$, but no significant day by cheese variable interaction $\left(\mathrm{F}_{7,1344}=\right.$ $0.4788, \mathrm{P}=0.8504)$ was observed. The two factor analysis for average salty liking revealed a significant main effect for day $\left(\mathrm{F}_{1,1344}=8.82, \mathrm{P}=0.0030\right)$ and cheese variable $\left(\mathrm{F}_{7,1344}=53.53, \mathrm{P}<0.0001\right)$, but no significant day by cheese variable interaction $\left(\mathrm{F}_{7,1344}\right.$ $=0.6339, \mathrm{P}=0.7281)$. The two factor analysis for average texture liking indicated a significant main effect for cheese variable $\left(\mathrm{F}_{7,1344}=47.5, \mathrm{P}<0.0001\right)$, but no significant main effects for day $\left(\mathrm{F}_{1,1344}=1.60, \mathrm{P}=0.2059\right)$ or day by cheese variable interaction $\left(\mathrm{F}_{7}\right.$, $1344=0.1622, \mathrm{P}=0.9923)$ were observed. The factorial analysis for average appearance liking indicated a significant main effect for cheese variable $\left(\mathrm{F}_{7,1344}=12.37, \mathrm{P}<0.0001\right)$, but no significant main effect for day $\left(\mathrm{F}_{1,1344}=2.6019, \mathrm{P}=0.1070\right)$ or significant interaction $\left(\mathrm{F}_{7,1344}=0.7347, \mathrm{P}=0.6424\right)$ were observed. The two factor analysis for average aroma liking revealed a significant main effect for day $\left(\mathrm{F}_{1,1344}=16.39\right.$, $\mathrm{P}<0.0001)$ and cheese variable $\left(\mathrm{F}_{7,1344}=21.77, \mathrm{P}<0.0001\right)$, but no significant day by cheese variable interaction $\left(\mathrm{F}_{7,1344}=1.57, \mathrm{P}=0.1400\right)$ was observed. When a significant main effect for day was observed, the first day of testing had a higher mean score compared to the second day of testing (Table 5.9). 
Table 5.9. Average Liking Day 1 versus Average Liking Day 2. Mean and standard errors for each significant average liking attribute; for both days of testing, first day scores are higher than second day scores.

\begin{tabular}{|c|c|c|}
\hline \multicolumn{3}{|c|}{ Average Liking Day 1 versus Average Liking Day 2} \\
Day 1 and Day 2 \\
\hline Liking Attribute & Day 1 Mean \pm Std Err & Day 2 Mean \pm Std Err \\
\hline Overall Flavor & $5.29 \pm 0.074$ & $4.92 \pm 0.077$ \\
\hline Saltiness & $5.18 \pm 0.069$ & $4.93 \pm 0.072$ \\
\hline Aroma & $5.96 \pm 0.053$ & $5.66 \pm 0.056$ \\
\hline
\end{tabular}

\subsubsection{Comparison of Each Change in Emotion by Cheese Variable}

The change in emotion comparison across each cheese variable for the first day of testing yielded significant Kruskal-Wallis tests: caring $\left(\mathrm{Chi}-\mathrm{Sq}_{7}=33.51, \mathrm{P}<0.0001\right)$, excited $\left(\mathrm{Chi}-\mathrm{Sq}_{7}=22.59, \mathrm{P}=0.0020\right)$, self-confident $\left(\mathrm{Chi}-\mathrm{Sq}_{7}=17.57, \mathrm{P}=0.0141\right)$, fatigued $\left(\mathrm{Chi}-\mathrm{Sq}_{7}=15.38, \mathrm{P}=0.0314\right)$, judgmental $\left(\mathrm{Chi}-\mathrm{Sq}_{7}=23.59, \mathrm{P}=0.0013\right)$, and $\mathrm{sad}$ $\left(\mathrm{Chi}-\mathrm{Sq}_{7}=28.88, \mathrm{P}=0.0002\right)$. The Steel-Dwass all pairs nonparametric post hoc comparisons for caring, excited, self-confident, fatigued, judgmental, and sad can be observed in Tables 5.10, 5.11, 5.12, 5.13, 5.14, and 5.15 respectively; variables with different letters are significantly different from one another (5\%).

Table 5.10. Change in Caring by Variable Day 1. Post hoc comparison of the change in caring across all cheese variables, including an adjusted mean value for consideration given the use of the Kruskal-Wallis test.

\begin{tabular}{|c|c|c|c|c|}
\hline \multicolumn{5}{|c|}{$\begin{array}{c}\text { Change in Caring by Variable } \\
\text { Day 1 }\end{array}$} \\
\hline Variable & Mean & \multicolumn{1}{c|}{ (Mean-Mean0)/Std0 } & \multicolumn{1}{c|}{ SD } & Differences \\
\hline Full Sodium & 0.164 & 4.926 & 0.403 & A \\
\hline $100 \%$ Salona & 0.047 & 1.437 & 0.405 & A B \\
\hline $50 \%$ Salona & 0.011 & 0.392 & 0.361 & A B \\
\hline $50 \% \mathrm{KCl}$ & -0.023 & -0.652 & 0.219 & B \\
\hline $100 \% \mathrm{KCl}$ & -0.023 & -0.654 & 0.344 & B \\
\hline $50 \%$ Alta & -0.035 & -1.001 & 0.241 & B \\
\hline Low Sodium & -0.071 & -2.048 & 0.257 & B \\
\hline $100 \%$ Alta & -0.082 & -2.398 & 0.316 & B \\
\hline
\end{tabular}


Table 5.11. Change in Excited by Variable Day 1. Post hoc comparison of the change in excited across all cheese variables, including an adjusted mean value for consideration given the use of the KruskalWallis test.

\begin{tabular}{|c|c|c|c|c|}
\hline \multicolumn{5}{|c|}{$\begin{array}{c}\text { Change in Excited by Variable } \\
\text { Day } 1\end{array}$} \\
\hline Variable & Mean & (Mean-Mean0)/Std0 & SD & Differences \\
\hline Full Sodium & 0.082 & 3.428 & 0.581 & $\mathrm{~A}$ \\
\hline $50 \% \mathrm{KCl}$ & -0.024 & 1.451 & 0.533 & A $\mathrm{B}$ \\
\hline $100 \%$ Salona & -0.082 & 0.435 & 0.414 & A B \\
\hline $100 \% \mathrm{KCl}$ & -0.094 & 0.064 & 0.548 & A $B$ \\
\hline $50 \%$ Alta & -0.129 & -0.446 & 0.371 & A $\mathrm{B}$ \\
\hline $100 \%$ Alta & -0.129 & -0.577 & 0.506 & A $\mathrm{B}$ \\
\hline $50 \%$ Salona & -0.164 & -1.244 & 0.484 & A B \\
\hline Low Sodium & -0.259 & -3.111 & 0.491 & $\mathrm{~B}$ \\
\hline
\end{tabular}

Table 5.12. Change in Self Confident by Variable Day 1. Post hoc comparison of the change in selfconfident across all cheese variables, including an adjusted mean value for consideration given the use of the Kruskal-Wallis test.

\begin{tabular}{|c|c|c|c|l|}
\hline \multicolumn{7}{|c|}{$\begin{array}{c}\text { Change in Self Confident by Variable } \\
\text { Day 1 }\end{array}$} \\
\hline Variable & Mean & \multicolumn{1}{c|}{ (Mean-Mean0)/Std0 } & \multicolumn{1}{c|}{ SD } & Differences \\
\hline Full Sodium & 0.047 & 2.622 & 0.46 & A \\
\hline $100 \%$ KCl & 0.000 & 1.536 & 0.463 & A B \\
\hline $100 \%$ Salona & -0.035 & 0.751 & 0.421 & A B \\
\hline 100\% Alta & -0.059 & 0.228 & 0.388 & A B \\
\hline 50\% KCl & -0.082 & -0.334 & 0.414 & A B \\
\hline 50\% Salona & -0.094 & -0.616 & 0.426 & A B \\
\hline Low Sodium & -0.129 & -1.4 & 0.371 & A B \\
\hline 50\% Alta & -0.188 & -2.788 & 0.393 & B \\
\hline
\end{tabular}

Table 5.13. Change in Fatigued by Variable Day 1. Post hoc comparison of the change in fatigued across all cheese variables, including an adjusted mean value for consideration given the use of the Kruskal-Wallis test.

\begin{tabular}{|c|c|c|c|c|c|}
\hline \multicolumn{7}{|c|}{$\begin{array}{c}\text { Change in Fatigued by Variable } \\
\text { Day 1 }\end{array}$} \\
\hline Variable & Mean & \multicolumn{1}{c|}{ (Mean-Mean0)/Std0 } & \multicolumn{1}{c|}{ SD } & Differences \\
\hline $100 \%$ KCl & 0.059 & 2.229 & 0.472 & A \\
\hline $50 \%$ Salona & 0.012 & 1.281 & 0.449 & A & B \\
\hline $50 \%$ Alta & -0.035 & 0.319 & 0.448 & A & B \\
\hline $50 \%$ KCl & -0.035 & 0.292 & 0.498 & A & B \\
\hline Low Sodium & -0.035 & 0.251 & 0.566 & A & B \\
\hline 100\% Alta & -0.059 & -0.161 & 0.446 & A & B \\
\hline Full Sodium & -0.106 & -1.150 & 0.489 & A & B \\
\hline 100\% Salona & -0.200 & -3.061 & 0.431 & B \\
\hline
\end{tabular}


Table 5.14. Change in Judgmental by Variable Day 1. Post hoc comparison of the change in judgmental across all cheese variables, including an adjusted mean value for consideration given the use of the Kruskal-Wallis test.

\begin{tabular}{|c|c|c|c|l|}
\hline \multicolumn{5}{|c|}{$\begin{array}{c}\text { Change in Judgmental by Variable } \\
\text { Day 1 }\end{array}$} \\
\hline Variable & Mean & \multicolumn{1}{c|}{ (Mean-Mean0)/Std0 } & \multicolumn{1}{c|}{ DD } & Differences \\
\hline Low Sodium & 0.224 & 3.268 & 0.497 & A \\
\hline 100\% Alta & 0.129 & 1.148 & 0.483 & A B \\
\hline $50 \%$ Alta & 0.118 & 0.854 & 0.447 & A B \\
\hline $50 \%$ KCl & 0.105 & 0.560 & 0.409 & A B \\
\hline 100\% Salona & 0.094 & 0.358 & 0.478 & A B \\
\hline $50 \%$ Salona & 0.012 & -1.514 & 0.422 & A B \\
\hline 100\% KCl & 0.000 & -1.807 & 0.378 & B \\
\hline Full Sodium & -0.047 & -2.868 & 0.342 & B \\
\hline
\end{tabular}

Table 5.15. Change in Sad by Variable Day 1. Post hoc comparison of the change in sad across all cheese variables, including an adjusted mean value for consideration given the use of the Kruskal-Wallis test.

\begin{tabular}{|c|c|c|c|c|}
\hline \multicolumn{7}{|c|}{$\begin{array}{c}\text { Change in Sad by Variable } \\
\text { Day 1 }\end{array}$} \\
\hline Variable & Mean & \multicolumn{1}{c|}{ (Mean-Mean0)/Std0 } & \multicolumn{1}{c|}{ SD } & Differences \\
\hline Low Sodium & 0.188 & 4.204 & 0.393 & A \\
\hline 100\% Alta & 0.094 & 1.207 & 0.332 & A B \\
\hline $50 \%$ Alta & 0.082 & 0.830 & 0.317 & A B \\
\hline 100\% Salona & 0.071 & 0.474 & 0.338 & A B \\
\hline 100\% KCl & 0.035 & -0.657 & 0.286 & A B \\
\hline 50\% Salona & 0.000 & -1.789 & 0.218 & B \\
\hline Full Sodium & 0.000 & -1.811 & 0.154 & B \\
\hline $50 \% \mathrm{KCl}$ & -0.024 & -2.458 & 0.344 & B \\
\hline
\end{tabular}

Fewer changes in emotion were significant across the eight cheese variables in the second day of testing, with the only significant comparisons being: aring $\left(\mathrm{Chi}-\mathrm{Sq}_{7}=\right.$ 23.86, $\mathrm{P}=0.0012)$ (Table 5.16), excited $\left(\mathrm{Chi}_{-\mathrm{Sq}} 7=20.22, \mathrm{P}=0.0051\right)$ (Table 5.17), sociable $($ Chi-Sq $7=19.09, \mathrm{P}=0.0079)\left(\right.$ Table 5.18), self-confident $\left(\mathrm{Chi}-\mathrm{Sq}_{7}=19.81\right.$, $\mathrm{P}=0.0060)$ (Table 5.19), judgmental $\left(\mathrm{Chi}-\mathrm{Sq}_{7}=28.30, \mathrm{P}=0.0002\right)$ (Table 5.20), and raging $\left(\mathrm{Chi}-\mathrm{Sq}_{7}=14.46, \mathrm{P}=0.0146\right)$ (Table 5.21). Tables 5.16 through 5.21 contain the 
nonparametric post hoc comparisons for each of the respective changes in emotions;

variables with different letters are significantly different (5\%).

Table 5.16. Change in Caring by Variable Day 2. Post hoc comparison of the change in caring across all cheese variables, including an adjusted mean value for consideration given the use of the Kruskal-Wallis test.

\begin{tabular}{|c|c|c|c|l|}
\hline \multicolumn{7}{|c|}{$\begin{array}{c}\text { Change in Caring by Variable } \\
\text { Day 2 }\end{array}$} \\
\hline Variable & Mean & \multicolumn{1}{l|}{ (Mean-Mean0)/Std0 } & \multicolumn{1}{c|}{ DD } \\
\hline Full Sodium & 0.114 & 3.094 & 0.369 & A \\
\hline $50 \%$ Salona & 0.082 & 2.243 & 0.277 & A \\
\hline $100 \%$ KCl & 0.016 & 0.531 & 0.386 & A B \\
\hline $100 \%$ Alta & 0.000 & 0.110 & 0.183 & A B \\
\hline $50 \%$ KCl & -0.016 & -0.320 & 0.341 & A B \\
\hline $100 \%$ Salona & -0.049 & -1.175 & 0.338 & A B \\
\hline $50 \%$ Alta & -0.065 & -1.599 & 0.249 & B \\
\hline Low Sodium & -0.114 & -2.883 & 0.321 & B \\
\hline
\end{tabular}

Table 5.17. Change in Excited by Variable Day 2. Post hoc comparison of the change in excited across all cheese variables, including an adjusted mean value for consideration given the use of the Kruskal-Wallis test.

\begin{tabular}{|c|c|c|c|c|}
\hline \multicolumn{5}{|c|}{$\begin{array}{c}\text { Change in Excited by Variable } \\
\text { Day } 2\end{array}$} \\
\hline Variable & Mean & (Mean-Mean0)/Std0 & SD & Differences \\
\hline $50 \% \mathrm{KCl}$ & 0.065 & 2.663 & 0.478 & A \\
\hline Full Sodium & 0.032 & 2.086 & 0.482 & A $B$ \\
\hline $100 \%$ Salona & 0.000 & 1.482 & 0.516 & A $\mathrm{B}$ \\
\hline $100 \% \mathrm{KCl}$ & -0.114 & -0.521 & 0.486 & A B \\
\hline $50 \%$ Salona & -0.131 & -0.768 & 0.427 & A B \\
\hline $100 \%$ Alta & -0.147 & -1.098 & 0.477 & A $\mathrm{B}$ \\
\hline $50 \%$ Alta & -0.196 & -1.921 & 0.401 & $\mathrm{~B}$ \\
\hline Low Sodium & -0.196 & -1.921 & 0.401 & $\mathrm{~B}$ \\
\hline
\end{tabular}


Table 5.18. Change in Sociable by Variable Day 2. Post hoc comparison of the change in sociable across all cheese variables, including an adjusted mean value for consideration given the use of the KruskalWallis test.

\begin{tabular}{|c|c|c|c|c|}
\hline \multicolumn{7}{|c|}{$\begin{array}{c}\text { Change in Sociable by Variable } \\
\text { Day 2 }\end{array}$} \\
\hline Variable & Mean & \multicolumn{1}{c|}{ (Mean-Mean0)/Std0 } & \multicolumn{1}{c|}{ SD } & \multicolumn{1}{c|}{ Differences } \\
\hline $50 \% \mathrm{KCl}$ & -0.082 & 2.741 & 0.420 & A \\
\hline $100 \% \mathrm{KCl}$ & -0.180 & 1.069 & 0.500 & A B \\
\hline Full Sodium & -0.180 & 1.069 & 0.500 & A B \\
\hline 100\% Salona & -0.180 & 0.998 & 0.532 & A B \\
\hline 50\% Alta & -0.295 & -0.737 & 0.494 & A B \\
\hline 50\% Salona & -0.295 & -0.880 & 0.558 & A B \\
\hline Low Sodium & -0.344 & -1.573 & 0.513 & A B \\
\hline 100\% Alta & -0.409 & -2.687 & 0.528 & B \\
\hline
\end{tabular}

Table 5.19. Change in Self Confident by Variable Day 2. Post hoc comparison of the change in sociable across all cheese variables, including an adjusted mean value for consideration given the use of the Kruskal-Wallis test.

\begin{tabular}{|c|c|c|c|l|}
\hline \multicolumn{7}{|c|}{$\begin{array}{c}\text { Change in Self Confident by Variable } \\
\text { Day 2 }\end{array}$} \\
\hline Variable & Mean & (Mean-Mean0)/Std0 & SD & Differences \\
\hline Full Sodium & 0.114 & 2.963 & 0.412 & A \\
\hline $100 \% \mathrm{KCl}$ & 0.049 & 1.644 & 0.425 & A B \\
\hline $50 \% \mathrm{KCl}$ & 0.032 & 1.321 & 0.401 & A B \\
\hline $50 \%$ Alta & -0.049 & -0.308 & 0.338 & A B \\
\hline $100 \%$ Salona & -0.066 & -0.656 & 0.403 & A B \\
\hline $50 \%$ Salona & -0.082 & -0.991 & 0.420 & A B \\
\hline 100\% Alta & -0.131 & -1.998 & 0.465 & A B \\
\hline Low Sodium & -0.131 & -1.974 & 0.386 & B \\
\hline
\end{tabular}


Table 5.20. Change in Judgmental by Variable Day 2. Post hoc comparison of the change in judgmental across all cheese variables, including an adjusted mean value for consideration given the use of the

Kruskal-Wallis test.

\begin{tabular}{|c|c|c|c|l|}
\hline \multicolumn{7}{|c|}{$\begin{array}{c}\text { Change in Judgmental by Variable } \\
\text { Day 2 }\end{array}$} \\
\hline Variable & Mean & \multicolumn{1}{c|}{ (Mean-Mean0)/Std0 } & \multicolumn{1}{c|}{ SD } & \multicolumn{1}{c|}{ Differences } \\
\hline Low Sodium & 0.279 & 4.111 & 0.521 & A \\
\hline $100 \%$ Alta & 0.148 & 1.531 & 0.401 & A B \\
\hline $50 \%$ KCl & 0.098 & 0.639 & 0.473 & A B \\
\hline $50 \%$ Alta & 0.081 & 0.274 & 0.378 & A B \\
\hline $100 \%$ KCl & 0.016 & -0.958 & 0.387 & A B \\
\hline $50 \%$ Salona & 0.000 & -1.278 & 0.365 & A B \\
\hline $100 \%$ Salona & -0.016 & -1.553 & 0.427 & A B \\
\hline Full Sodium & -0.082 & -2.765 & 0.458 & B \\
\hline
\end{tabular}

Table 5.21. Change in Raging by Variable Day 2. Post hoc comparison of the change in raging across all cheese variables, including an adjusted mean value for consideration given the use of the Kruskal-Wallis test. Note: although raging did have a significant global test, the post hoc comparison did not indicate any differences between samples.

\begin{tabular}{|c|c|c|c|l|}
\hline \multicolumn{7}{|c|}{$\begin{array}{c}\text { Change in Raging by Variable } \\
\text { Day 2 }\end{array}$} \\
\hline Variable & Mean & \multicolumn{1}{c|}{ (Mean-Mean0)/Std0 } & \multicolumn{1}{c|}{ SD } & Differences \\
\hline 100\% Alta & 0.114 & 2.749 & 0.321 & $\mathrm{~A}$ \\
\hline Low Sodium & 0.098 & 2.167 & 0.300 & $\mathrm{~A}$ \\
\hline 50\% Alta & 0.049 & 0.443 & 0.284 & $\mathrm{~A}$ \\
\hline $50 \%$ Salona & 0.032 & -0.136 & 0.256 & $\mathrm{~A}$ \\
\hline 50\% KCl & 0.016 & -0.718 & 0.223 & $\mathrm{~A}$ \\
\hline Full Sodium & 0.000 & -1.321 & 0.000 & $\mathrm{~A}$ \\
\hline $100 \%$ Salona & 0.000 & -1.321 & 0.000 & $\mathrm{~A}$ \\
\hline $100 \% \mathrm{KCl}$ & -0.016 & -1.860 & 0.223 & $\mathrm{~A}$ \\
\hline
\end{tabular}

\subsubsection{Comparison of First Emotion Scores to End Emotion Scores}

In order to evaluate subject emotions, each initial emotion was compared to the appropriate final emotional responses, the following emotions changed significantly across the consumption experience: excited $(S=-3043.0, P<0.0001)$, sociable $(S=-994.5$, $\mathrm{P}=0.0345)$, self-confident $(\mathrm{S}=-1437.5, \mathrm{P}<0.0001)$, surprise $(\mathrm{S}=2397.5, \mathrm{P}<0.0001)$, 
fatigued $(S=-1351.5, P=0.0064)$, judgmental $(S=1849.5, P<0.0001)$, raging $(S=168.0$, $\mathrm{P}<0.0001)$, sad $(\mathrm{S}=655.5, \mathrm{P}<0.0001)$, and fear $(\mathrm{S}=-246.5, \mathrm{P}=0.0230)$. Table 5.22 contains the initial and end emotion means, standard errors, and p-values for the first day of testing.

The initial and end emotional responses for each emotion were compared for the second day of testing, and emotions which changed significantly included: excited $(\mathrm{S}=$ 1165.5, $\mathrm{P}<0.0001)$, sociable $(\mathrm{S}=-4770.0, \mathrm{P}<0.0001)$, surprise $(\mathrm{S}=1287.0, \mathrm{P}<0.0001)$, judgmental $(S=776.0, P=0.0008), \operatorname{raging}(S=130.5, P=0.0002), \operatorname{sad}(S=345.0$, $\mathrm{P}=0.0021)$, and fear $(\mathrm{S}=-211.5, \mathrm{P}=0.0065)$. Table 5.23 contains the initial and end emotion means, standard errors, and p-values for the second day of testing.

Table 5.22. Change in Emotion: First versus End Day 1. Comparison of the first and last emotional responses for day 1 for all emotions; emotions which changed significantly are bolded

\begin{tabular}{|c|c|c|c|c|}
\hline \multicolumn{5}{|c|}{ Change in Emotion: First versus End } \\
\hline Emotion & First Mean & End Mean & Std Error & p-value \\
\hline Caring & 0.123 & 0.122 & 0.013 & 0.9090 \\
\hline Excited & $\mathbf{0 . 3 9 4}$ & $\mathbf{0 . 2 9 4}$ & $\mathbf{0 . 0 1 9}$ & $<\mathbf{0 . 0 0 0 1}$ \\
\hline Sociable & $\mathbf{0 . 2 6 2}$ & $\mathbf{0 . 2 2 4}$ & $\mathbf{0 . 0 1 8}$ & $\mathbf{0 . 0 3 4 5}$ \\
\hline Self Confident & $\mathbf{0 . 2 5 6}$ & $\mathbf{0 . 1 8 8}$ & $\mathbf{0 . 0 1 6}$ & $<\mathbf{0 . 0 0 0 1}$ \\
\hline Surprise & $\mathbf{0 . 0 8 2}$ & $\mathbf{0 . 1 8 5}$ & $\mathbf{0 . 0 1 7}$ & $<\mathbf{0 . 0 0 0 1}$ \\
\hline Detached & 0.224 & 0.219 & 0.017 & 0.8016 \\
\hline Fatigued & $\mathbf{0 . 3 1 2}$ & $\mathbf{0 . 2 6 2}$ & $\mathbf{0 . 0 1 8}$ & $\mathbf{0 . 0 0 6 4}$ \\
\hline Inferior & 0.026 & 0.035 & 0.009 & 0.2643 \\
\hline Judgmental & $\mathbf{0 . 1 7 1}$ & $\mathbf{0 . 2 5 0}$ & $\mathbf{0 . 0 1 7}$ & $<\mathbf{0 . 0 0 0 1}$ \\
\hline Raging & $\mathbf{0 . 0 1 3}$ & $\mathbf{0 . 0 4 4}$ & $\mathbf{0 . 0 0 8}$ & $<\mathbf{0 . 0 0 0 1}$ \\
\hline Sad & $\mathbf{0 . 0 4 7}$ & $\mathbf{0 . 1 0 3}$ & $\mathbf{0 . 0 1 2}$ & $<\mathbf{0 . 0 0 0 1}$ \\
\hline Fear & $\mathbf{0 . 0 7 1}$ & $\mathbf{0 . 0 4 6}$ & $\mathbf{0 . 0 1 1}$ & $\mathbf{0 . 0 2 3 0}$ \\
\hline None of these & 0.087 & 0.112 & 0.014 & 0.0713 \\
\hline & & & &
\end{tabular}$? ? ? ?$


Table 5.23. Change in Emotion: First versus End Day 2. Comparison of the first and last emotional responses for day 2 for all emotions; emotions which changed significantly are bolded.

\begin{tabular}{|c|c|c|c|c|}
\hline \multicolumn{7}{|c|}{ Change in Emotion: First versus End } \\
\hline Day 2 \\
\hline Caring & 0.121 & 0.117 & 0.015 & 0.7805 \\
\hline Excited & $\mathbf{0 . 3 7 5}$ & $\mathbf{0 . 2 8 9}$ & $\mathbf{0 . 0 2 1}$ & $<\mathbf{0 . 0 0 0 1}$ \\
\hline Sociable & $\mathbf{0 . 4 8 4}$ & $\mathbf{0 . 2 3 8}$ & $\mathbf{0 . 0 2 3}$ & $<\mathbf{0 . 0 0 0 1}$ \\
\hline Self Confident & 0.258 & 0.225 & 0.019 & 0.0808 \\
\hline Surprise & $\mathbf{0 . 0 6 4}$ & $\mathbf{0 . 1 7 0}$ & $\mathbf{0 . 0 2 0}$ & $<\mathbf{0 . 0 0 0 1}$ \\
\hline Detached & 0.215 & 0.205 & 0.017 & 0.5566 \\
\hline Fatigued & 0.369 & 0.334 & 0.019 & 0.0681 \\
\hline Inferior & 0.061 & 0.053 & 0.012 & 0.5009 \\
\hline Judgmental & $\mathbf{0 . 1 5 2}$ & $\mathbf{0 . 2 1 7}$ & $\mathbf{0 . 0 2 0}$ & $\mathbf{0 . 0 0 0 8}$ \\
\hline Raging & $\mathbf{0 . 0 2 0}$ & $\mathbf{0 . 0 5 7}$ & $\mathbf{0 . 0 1 1}$ & $\mathbf{0 . 0 0 0 2}$ \\
\hline Sad & $\mathbf{0 . 0 6 6}$ & $\mathbf{0 . 1 1 2}$ & $\mathbf{0 . 0 1 6}$ & $\mathbf{0 . 0 0 2 1}$ \\
\hline Fear & $\mathbf{0 . 0 8 2}$ & $\mathbf{0 . 0 4 5}$ & $\mathbf{0 . 0 1 4}$ & $\mathbf{0 . 0 0 6 5}$ \\
\hline None of these & 0.086 & 0.068 & 0.010 & 0.0708 \\
\hline
\end{tabular}

\subsubsection{Emotional Changes between Day 1 and Day 2}

Each change (end - first) in emotion from the first day of testing, was compared to corresponding emotional change from the second day of testing, four emotional changes differed between the two days of testing: sociable ( $\mathrm{S}=-300137, \mathrm{P}<0.0001)$, selfconfident ( $\mathrm{S}=31280.0, \mathrm{P}=0.0014)$, detached $(\mathrm{S}=-25251, \mathrm{P}=0.0093)$, fatigued $(\mathrm{S}=31201$, $\mathrm{P}=0.0080)$, inferior $(\mathrm{S}=-6687.5, \mathrm{P}=0.0009)$, judgmental $(\mathrm{S}=-25140, \mathrm{P}=0.0099)$, sad $(\mathrm{S}=$ -12107, $\mathrm{P}=0.0333)$, and none of these $(\mathrm{S}=-14973, \mathrm{P}<0.0001)$. Table 5.24 contains the emotion means from the first and second day of testing, the standard error, and p-values for each of the emotions. 
Table 5.24. Comparing Day 1 Emotion Changes to Day 2 Emotion Changes. Comparison of the change in emotion between day 1 and day 2 of testing; emotions which differed significantly are bolded.

\begin{tabular}{|c|c|c|c|c|}
\hline \multicolumn{5}{|c|}{ Comparing Day 1 Emotion Changes to Day 2 Emotion Changes } \\
Days 1 and 2 \\
\hline Emotion & 1st Day Mean & 2nd Day Mean & Std Error & p-value \\
\hline Caring & 0.002 & -0.004 & 0.007 & 0.4042 \\
\hline Excited & -0.094 & -0.086 & 0.011 & 0.4321 \\
\hline Sociable & $\mathbf{- 0 . 0 4 1}$ & $\mathbf{- 0 . 2 4 6}$ & $\mathbf{0 . 0 1 1}$ & $<\mathbf{0 . 0 0 0 1}$ \\
\hline Self Confident & $\mathbf{- 0 . 0 6 4}$ & $\mathbf{- 0 . 0 3 3}$ & $\mathbf{0 . 0 1 0}$ & $\mathbf{0 . 0 0 1 4}$ \\
\hline Surprise & 0.100 & 0.107 & 0.010 & 0.5320 \\
\hline Detached & $\mathbf{0 . 0 1 4}$ & $\mathbf{- 0 . 0 1 0}$ & $\mathbf{0 . 0 0 9}$ & $\mathbf{0 . 0 0 9 3}$ \\
\hline Fatigued & $\mathbf{- 0 . 0 6 2}$ & $\mathbf{- 0 . 0 3 5}$ & $\mathbf{0 . 0 1 0}$ & $\mathbf{0 . 0 0 8 0}$ \\
\hline Inferior & $\mathbf{0 . 0 1 0}$ & $\mathbf{- 0 . 0 0 8}$ & $\mathbf{0 . 0 0 6}$ & $\mathbf{0 . 0 0 0 9}$ \\
\hline Judgmental & $\mathbf{0 . 0 9 0}$ & $\mathbf{0 . 0 6 6}$ & $\mathbf{0 . 0 0 9}$ & $\mathbf{0 . 0 0 9 9}$ \\
\hline Raging & 0.034 & 0.037 & 0.005 & 0.6737 \\
\hline Sad & $\mathbf{0 . 0 6 4}$ & $\mathbf{0 . 0 4 7}$ & $\mathbf{0 . 0 0 8}$ & $\mathbf{0 . 0 3 3 3}$ \\
\hline Fear & -0.033 & -0.037 & 0.007 & 0.5483 \\
\hline None of these & $\mathbf{0 . 0 1 2}$ & $\mathbf{- 0 . 0 1 8}$ & $\mathbf{0 . 0 0 6}$ & $<\mathbf{0 . 0 0 0 1}$ \\
\hline
\end{tabular}

\subsubsection{Comparison of Change in Emotion by Gender}

The change in emotion was compared between males and females for both days of testing. The MANOVA test for the first day of testing was significant $\left(\mathrm{F}_{13,666}=2.50\right.$, $\mathrm{P}=0.0024)$ and the only differences in emotional changes between males and females were surprise $\left(\mathrm{Chi}-\mathrm{Sq}_{1}=10.46, \mathrm{P}=0.0012\right)$, inferior $\left(\mathrm{Chi}-\mathrm{Sq}_{1}=6.27, \mathrm{P}=0.0123\right)$, $\mathrm{sad}$ $\left(\right.$ Chi-Sq $\left.{ }_{1}=5.50, \mathrm{P}=0.0190\right)$, and "none of these" $\left(\right.$ Chi-Sq $\left.{ }_{1}=4.08, \mathrm{P}=0.0433\right)$ on the first day of testing. The MANOVA for the second day of testing did not yield a significant whole model effect $\left(\mathrm{F}_{13,474}=1.73, \mathrm{P}=0.0528\right)$, thus further investigation was not appropriate; however, considering the p-value was relatively close to significance, exploration of any significant emotions indicated a significant difference in change in judgmental $\left(\mathrm{F}_{1}=11.69, \mathrm{P}=0.0006\right)$ by gender. Table 5.25 and Table 5.26 indicate the means and standard errors for females and males for the significant emotion, first day surprise and second day judgmental, respectively. 
Table 5.25. Change in Emotions by Gender Day 1. Significant difference in change in the significant emotions between males and females, with males tending to have a greater positive change than females.

\begin{tabular}{|c|c|c|c|c|c|}
\hline \multicolumn{7}{|c|}{ Change in Emotions by Gender } \\
Day 1 \\
\hline Emotion & Female Mean & Female Std Error & Male Mean & Male Std Error & p-value \\
\hline Surprise & 0.073 & 0.019 & 0.200 & 0.034 & 0.0012 \\
\hline Inferior & -0.002 & 0.009 & 0.044 & 0.016 & 0.0123 \\
\hline Sad & 0.040 & 0.014 & 0.106 & 0.025 & 0.0190 \\
\hline None of these & 0.040 & 0.016 & -0.025 & 0.028 & 0.0433 \\
\hline
\end{tabular}

Thapbe 5.26. Change in Emotions by Gender Day 2. Significant difference in change in judgmental between males and females, with females having a greater positive change than males.

\begin{tabular}{|c|c|c|c|c|c|}
\hline \multicolumn{5}{|c|}{ Change in Emotions by Gender } \\
Day 2 \\
\hline Emotion & Female Mean & Female Std Error Male Mean Male Std Error p-value \\
\hline Judgmental & 0.102 & 0.022 & -0.067 & 0.043 & 0.0006 \\
\hline
\end{tabular}

\subsubsection{Texture analysis}

The normality assumption was not met for any of the tested texture attributes, thus the Kruskal-Wallis test was used for comparing all texture attributes by cheese variable. The following comparisons were significant $(\alpha=0.05)$ : hardness $\left(\mathrm{Chi}-\mathrm{Sq}_{7}=19.93, \mathrm{P}=\right.$ 0.0057), cohesiveness $\left(\mathrm{Chi}-\mathrm{Sq}_{7}=26.64, \mathrm{P}=0.0004\right)$, gumminess $\left(\mathrm{Chi}-\mathrm{Sq}{ }_{7}=19.97, \mathrm{P}=\right.$ 0.0056), and chewiness $\left(\mathrm{Chi}-\mathrm{Sq}_{7}=19.97, \mathrm{P}=0.0056\right)$. The Steel-Dwass all-pairs nonparametric post hoc comparisons for hardness, cohesiveness, gumminess, and chewiness can be observed in Tables 5.27, 5.28, 5.29, and 5.30, respectively; variables which did not share difference letters are significantly different from one another $(5 \%)$. Note: although the global p-values for each of the Kruskal-Wallis tests indicated significant differences between samples by each of the listed texture attributes, the post 
hoc comparison did not yield specific differences between the samples for any of the significant texture attributes.

Table 5.27. Hardness by Cheese Variable. Nonparametric post hoc comparison between cheese variables for hardness.

\begin{tabular}{|c|c|c|c|l|}
\hline \multicolumn{7}{|c|}{ Hardness by Cheese Variable } \\
\hline Variable & Mean & \multicolumn{1}{l|}{ (Mean-Mean0)/Std0 } & \multicolumn{1}{l|}{ SD } & \multicolumn{1}{l|}{ Differences } \\
\hline $50 \% \mathrm{KCl}$ & 3674.60 & 2.413 & 488.15 & $\mathrm{~A}$ \\
\hline $100 \%$ Salona & 3478.20 & 1.595 & 484.54 & $\mathrm{~A}$ \\
\hline $50 \%$ Salona & 3312.28 & 1.104 & 154.75 & $\mathrm{~A}$ \\
\hline $100 \%$ Alta & 3243.56 & 0.286 & 953.53 & $\mathrm{~A}$ \\
\hline Full Sodium & 3353.76 & -0.245 & 1383.50 & $\mathrm{~A}$ \\
\hline Low Sodium & 2950.06 & -0.327 & 406.59 & $\mathrm{~A}$ \\
\hline $50 \%$ Alta & 2633.32 & -1.759 & 421.81 & $\mathrm{~A}$ \\
\hline $100 \% \mathrm{KCl}$ & 2177.62 & -3.067 & 432.18 & $\mathrm{~A}$ \\
\hline
\end{tabular}

Table 5.28. Cohesiveness by Cheese Variable. Nonparametric post hoc comparison between cheese variables for cohesiveness.

Cohesiveness by Cheese Variable

\begin{tabular}{|c|c|c|c|c|}
\hline Variable & Mean & (Mean-Mean0)/Std0 & SD & Differences \\
\hline Full Sodium & 0.580 & 3.231 & 0.325 & A \\
\hline $100 \% \mathrm{KCl}$ & 0.385 & 1.963 & 0.019 & A \\
\hline $100 \%$ Salona & 0.522 & 1.636 & 0.341 & A \\
\hline $50 \%$ Salona & 0.335 & -0.245 & 0.037 & A \\
\hline $50 \% \mathrm{KCl}$ & 0.321 & -0.900 & 0.021 & A \\
\hline $100 \%$ Alta & 0.320 & -0.982 & 0.024 & A \\
\hline $50 \%$ Alta & 0.294 & -2.004 & 0.045 & A \\
\hline Low Sodium & 0.294 & -2.658 & 0.021 & A \\
\hline
\end{tabular}

Table 5.29. Gumminess by Cheese Variable. Nonparametric post hoc comparison between cheese variables for gumminess

\section{Gumminess by Cheese Variable}

\begin{tabular}{|c|c|c|c|l|}
\hline \multicolumn{1}{|c|}{ Variable } & \multicolumn{1}{c}{ Mean } & \multicolumn{1}{c|}{ Mean-Mean0)/Std0 } & SD & Differences \\
\hline $100 \%$ Salona & 1934.10 & 2.127 & 1588.250 & $\mathrm{~A}$ \\
\hline Full Sodium & 1958.50 & 1.922 & 1285.450 & $\mathrm{~A}$ \\
\hline $50 \% \mathrm{KCl}$ & 1182.76 & 1.268 & 205.640 & $\mathrm{~A}$ \\
\hline $50 \%$ Salona & 1108.88 & 0.818 & 126.670 & $\mathrm{~A}$ \\
\hline $100 \%$ Alta & 1050.88 & -0.204 & 368.320 & $\mathrm{~A}$ \\
\hline $100 \% \mathrm{KCl}$ & 839.88 & -1.840 & 175.470 & $\mathrm{~A}$ \\
\hline Low Sodium & 865.76 & -1.922 & 126.700 & $\mathrm{~A}$ \\
\hline $50 \%$ Alta & 789.97 & -2.167 & 233.460 & $\mathrm{~A}$ \\
\hline
\end{tabular}


Table 5.30. Chewiness by Cheese Variable. Nonparametric post hoc comparison between cheese variables for chewiness.

\begin{tabular}{|c|c|c|c|l|}
\hline \multicolumn{5}{|c|}{ Chewiness by Cheese Variable } \\
\hline Variable & \multicolumn{1}{|c|}{ Mean } & \multicolumn{1}{l|}{ (Mean-Mean0)/Std0 } & \multicolumn{1}{l|}{ SD } & Differences \\
\hline 100\% Salona & 966.81 & 2.127 & 794.24 & $\mathrm{~A}$ \\
\hline Full Sodium & 978.97 & 1.922 & 642.75 & $\mathrm{~A}$ \\
\hline $50 \% \mathrm{KCl}$ & 591.00 & 1.268 & 102.58 & $\mathrm{~A}$ \\
\hline $50 \%$ Salona & 554.08 & 0.818 & 63.21 & $\mathrm{~A}$ \\
\hline $100 \%$ Alta & 525.37 & -0.204 & 184.15 & $\mathrm{~A}$ \\
\hline $100 \% \mathrm{KCl}$ & 419.77 & -1.840 & 87.74 & $\mathrm{~A}$ \\
\hline Low Sodium & 432.62 & -1.922 & 63.43 & $\mathrm{~A}$ \\
\hline $50 \%$ Alta & 394.83 & -2.167 & 116.70 & $\mathrm{~A}$ \\
\hline
\end{tabular}

????The results of the salt analysis indicated the full sodium cheese sample contained approximately $1.61 \%$ salt, while the low sodium samples contained approximately $0.65 \%$ salt; these results suggest the salt retention of the performed method was approximately $90 \%$. The moisture analysis results indicated the full sodium cheese samples had a moisture content of $46.72 \%$ and the low sodium samples contained $47.83 \%$ moisture.

\subsection{Discussion}

Consumer acceptability of low sodium products has been a significant challenge for the food industry, and specifically the cheese industry, to overcome (Drake et al., 2011; Guinee, 2004). The current study took a multifaceted approach to understanding the consumer's relationship to low sodium mozzarella cheese by way of exploring the consumer's emotional and hedonic relationship to the low sodium cheese products, and investigated how emotions and liking changed across the eating and testing experiences. Texture profile analysis (TPA) was also performed to understand how sodium reduction in mozzarella cheese affected different texture attributes. It was hypothesized that 
products with higher salt content would have higher subject acceptability scores and would have a net positive change in the positive emotion and a net negative change in the negative emotions, while the low sodium cheeses were hypothesized to be less liked by subjects and decrease in positive emotions and increase in negative emotions over the testing experience; these hypotheses are based upon results of a previous study from the researchers (Collinsworth et al., 2013).

\subsubsection{Product Hedonics}

The results suggested the full sodium cheese was liked significantly more than all other variables in all liking attributes expect for appearance and aroma liking, where the $100 \% \mathrm{KCl}$ was not significantly different than the full sodium product (Table 5.3). The investigators were most interested in determining differences between samples in overall flavor, saltiness, and texture liking. For overall flavor liking, although the full sodium cheese was liked significantly more than all the other variables (Table 5.3), $100 \% \mathrm{KCl}$, $50 \% \mathrm{KCl}, 50 \%$ Salona, and $100 \%$ Salona did not have significantly different liking scores and their mean liking scores were all above 5.0, for the first day of testing (31 days post addition of salt replacers). Considering the 9-point hedonic scale, the " 5 " is considered "neither liked nor disliked", therefore these samples are not "disliked" and thus acceptable. The saltiness liking scores suggest the $100 \% \mathrm{KCl}$ variable, although liked less than the full sodium sample, had the next highest mean score and did not share difference letters with any of the lower mean (less than 5.0) scored samples (Table 5.3). The texture liking results indicated similar findings to the overall flavor liking results, suggesting $100 \% \mathrm{KCl}, 100 \%$ Salona, $50 \% \mathrm{KCl}$, and 50\% Salona had the next highest mean scores after the full sodium product, and their mean scores were all above 5.0 
(Table 5.3). Based upon the hedonic scores from the first day of testing, it appears the $100 \% \mathrm{KCl}$ cheese sample would be the most consumer acceptable "low sodium" cheese compared to the other tested cheeses variables.

The second day of testing results were similar to those of the first day, with $100 \%$ $\mathrm{KCl}$ consistently having the second highest mean scores after the full sodium variable; remarkably, the mean scores of the full sodium and $100 \% \mathrm{KCl}$ variables for saltiness liking did not differ significantly. Based upon both days of testing, the $100 \% \mathrm{KCl}$ sample appears to be the most appropriate sample for further investigation with consumer acceptability of low sodium cheese. These results are not surprising considering the favorable effects of using $\mathrm{KCl}$ for partial substitution of $\mathrm{NaCl}$ found in several other studies which investigated this relationship in different cheeses including: feta, cheddar, and imitation cheese (Katsiari et al., 1997; Grummer et al., 2012; El-Bakry et al., 2011). Although partial substitution of $\mathrm{NaCl}$ with $\mathrm{KCl}$ is promising, one potential setback of using $\mathrm{KCl}$ is the potential bitter aftertaste which is typically associable with $\mathrm{KCl}$ (Cruz et al., 2011; Grummer et al., 2012). The current study did not ask consumers about the potential "bitterness" or "aftertaste" of the samples, perhaps in future testing with these variables consumers should be ask to answer hedonic questions regarding "aftertaste" in order to understand the full consequences of using $\mathrm{KCl}$ as a $\mathrm{NaCl}$ substitute.

\subsubsection{Comparison of First, Middle, and End Liking Scores}

Observations in changes in liking score over the eating experience were of interest; thus hedonic scores were recorded at the first, halfway, and end of consuming each cheese product. The results from the first day of testing indicated a significant 
difference between the first (initial) and last (end) consumption liking scores for overall flavor (Table 5.5), saltiness (Table 5.6), texture (Table 5.7), and aroma liking (Table 5.8); additionally, the first liking time point was significantly higher than both the middle and end consumption for the texture and aroma hedonic scores. These results are not surprising considering what is known about sensory specific satiety (Rolls et al., 1981; Kringelbach, 2009) where subjects like a product less after consuming it compared to an uneaten product; however, a potential aftertaste effect of the salt replacers could also lead to similar results. The second day of testing did not yield significant results for change in liking for any of the testing hedonic attributes. Determining which day's results are more accurate is still unclear; however, the middle or halfway through consuming liking scores appear to be unnecessary, considering they were not significantly different from both the first and last hedonic scores. Further investigation of capturing first and last liking scores appears to have value.

\subsubsection{Changes in Liking Scores between Day 1 and Day 2}

The average for each liking attribute from the first day (31 days post addition of salt replacer) of testing was compared to the corresponding liking score for the second day of testing (51 days post addition of salt replacer), the results indicated the mean flavor, saltiness, and aroma liking attributes for the first day of testing were significantly higher than those from the second day of testing. Although, these results are somewhat expected considering subjects may prefer a fresher mozzarella cheese compared to an older mozzarella cheese, this information is relevant for manufacturers interested in making low sodium cheese. The samples used in the second day of testing were approximately seven weeks old and a significant reduction in liking is unacceptable 
considering the shelf-life for mozzarella cheese should be closer to 60-90 days post production. Considering the significant decrease in sensory liking between the two days of testing, perhaps the salt replacers and antimicrobial were not able to remain active for the length of time between the two days of testing or perhaps flavor and texture changes during the aging process resulted in undesirable outcomes. Additional investigation of extending the sensory quality shelf-life of low sodium cheeses is necessary for success of the product.

\subsubsection{Comparison of Each Change in Emotion by Cheese Variable}

Findings for the changes in emotion by each cheese variable mostly confirmed the investigator's hypotheses with the higher hedonic scored products having an increase in positive emotions over the eating experience, while lower hedonic scored products decreased in positive emotions over time. For change in caring (Table 5.10), the full sodium variable had significantly higher changes in caring compared to $100 \%$ ALTA $^{\mathrm{TM}}$ and the low sodium cheese (disliked products). Similarly, for change in excited (Table 5.11), the full sodium product had higher changes in excited over the eating experience compared to the low sodium variable; similar trends were seen in self-confident (Table 5.12). The reverse was seen for the negative emotions, judgmental (Table 5.14) and sad (Table 5.15), where disliked products such as the low sodium variable increased over the eating experience, while the more liked products such as the full sodium and $\mathrm{KCl}$ samples decreased in negative emotions over the eating experience. These trends were less clear for the fatigued emotion (Table 5.13), which may be due to it being a neutral emotion, and therefore it does not follow the trends seen in either the positive or negative emotions. 
Similar trends were found in the results from the second day of testing, with the full sodium cheese having a greater change in the positive emotions including: caring and self-confident, over time compared to the disliked products (Tables 5.16 and 5.19), and $50 \% \mathrm{KCl}$ (relatively high hedonic scores) had greater positive changes in excited (Table 5.17) and sociable (Table 5.18), both positive emotions. The low sodium cheeses had greater changes in the negative emotions, judgmental and raging (insignificant trend for raging) over time (Table 5.20 and 5.21). Considering the emotions were captured as categorical data, but treated as continuous the data should be interpreted with care; however, these findings are quite conservative and do show interesting trends with increases in positive emotions over time for liked products and increases in negative emotions overtime for disliked products. Additionally, when considering the analysis that was performed, the power was likely lower than if the emotions would have been analyzed as categorical, yet significant differences were still seen. Perhaps analyzing the current data differently would yield even more obvious emotion differences between the high and low hedonic scored products.

\subsubsection{Comparison of First Emotion Score to End Emotion Score}

When comparing the first emotional response to the end emotional response for each emotion, only certain emotions yielded a significant change. For first day of testing nine emotions changed significantly across the eating experience (Table 5.22), while only seven emotions changed significantly for the second day of testing (Table 5.23);

considering the emotions which did change significantly were very similar between the two days, perhaps these emotions are the most relevant for the product category. The emotions which did not change significantly overtime perhaps are not as relevant to low 
sodium cheese, and thus additional investigation of alternative emotion words could be pursued for this category.

\subsubsection{Emotional Changes between Day 1 and Day 2}

The changes in emotion for all emotions from the first day of testing were compared to the corresponding change in emotions from the second day of testing, and seven emotional changes and the "none of these" option differed significantly between the two days (Table 5.24); changes were relatively even across the different types of emotions (positive, neutral, and negative). These results are not surprising considering the significant changes in liking observed between the first and second day of testing, and the observed relationship between liking scores and changes in emotion. However, for the emotions which did not change significant, perhaps the subjects were more consistent in their emotional responses due to their poster board with the emotion images which may have kept them anchored to those certain emotions despite the testing dates occurring

three weeks apart; the consistency of the emotional responses is helpful for validation of the individualized poster board for future emotion testing research. Perhaps if liking scores did not change significantly between the two days of testing, more consistent emotional responses would have been observed.

\subsubsection{Comparison of Change in Emotion by Gender}

Although investigation of gender and emotion was not a major objective of this specific test, analysis was performed to compare the change in emotional response from males compared to females considering the cultural stereotype that women are "more emotional" than men. The results from the first day of testing suggested the only 
significant differences in change in emotion between men and women were in surprise, inferior, sad, and the "none of these" option (Table 5.25). Although the whole model MANOVA test for gender was not significant for the second day of testing, the only potentially significant emotion was judgmental (Table 5.26). Considering only three changes in emotion were significant between the 12 emotions tested across two days, there does not appear to be any major difference in gender when considering changes in emotion. Other studies suggested males and females likely experience the same emotions during a given stimuli; however, differences exist in expression and intensity of the emotional responses (Gard \& Kring, 2007; Kring \& Gordon, 1998). Several other studies have identified differences between men and women in their emotional responses to certain stimuli, including food (Costanzo et al., 1999; Macht, 1999; Seo et al., 2009); overall suggesting a difference in emotional responses between men and women. One limitation of the current study is the uneven number of males $(\sim 30 \%)$ and females $(\sim 70 \%)$, this difference in sample populations could affect the results, and thus the findings should be interpreted with caution.

\subsubsection{Texture Profile Analysis}

TPA was performed on all cheese variables after completion of the second day of testing. The samples differed significantly by hardness (Table 5.27), cohesiveness (Table 5.28), gumminess (Table 5.29), and chewiness (Table 5.30). Samples which were most preferred for overall flavor and saltiness liking (full sodium, $100 \% \mathrm{KCl}, 50 \% \mathrm{KCl}, 100 \%$ Salona, and 50\% Salona) appeared to have higher, but not statistically significant mean scores in the four significant texture attributes compared to the disliked samples (low sodium, $100 \% \mathrm{ALTA}^{\mathrm{TM}}$, and $50 \% \mathrm{ALTA}^{\mathrm{TM}}$ ). These results are noteworthy considering 
the only differences between the samples were the salt replacers, thus the different salt replacers resulted in difference texture measurements; findings similar to these results were anticipated. Texture Profile Analysis has traditionally been correlated with descriptive sensory results, specifically Spectrum ${ }^{\mathrm{TM}}$ method descriptive analysis (Bourne, 1978; Pons \& Fiszman, 1996; Murray et al., 2001); however, limited published information is available comparing the texture attribute profile of a low sodium cheese compared to the consumer liking scores. This information is valuable to manufacturers looking to produce a consumer acceptable low sodium cheese, because the importance of these texture attributes and their relationship to consumer acceptability is better understood.

\subsubsection{Main Study Objectives and Outcomes}

One major objective of the current study was to determine a salt replacer which was consistently acceptable and liked closely to a full sodium control cheese sample. Based upon the results of this study, the $100 \% \mathrm{KCl}$ bacteria cultured cheese variable (dipped in $0.275 \%$ SEA-i F75) appears to be the most appropriate for further investigation within mozzarella cheese. The $100 \% \mathrm{KCl}$ sample was a close to a 1:1 ratio of $\mathrm{NaCl}$ to $\mathrm{KCl}(100 \mathrm{~g} \mathrm{NaCl}: 81 \mathrm{~g} \mathrm{KCl})$, other studies have suggested this ratio appears to be the most effective at reducing sodium while still maintaining the sensory and functional properties of the cheese (El-Bakry et al., 2011; Cruz et al., 2011; Guinee, 2004; Grummer et al., 2012; Katsiari et al., 1997; Ayyash \& Shah, 2011). The tested cheese contained $47.83 \%$ moisture and $0.65 \%$ salt (salt contains approximately $39 \%$ sodium); meeting the moisture parameters for a LMPS mozzarella cheese and the sodium content for a low sodium cheese. In order to be a low sodium cheese as defined by the 
Code of Federal Regulations, a 30g sample (or 50g sample if reference amount customarily consumed is less than $30 \mathrm{~g}$ ) must contain less than $140 \mathrm{mg}$ of sodium (Mozzarella cheese and scamorza cheese, 1993), considering the tested cheese contained $0.65 \%$ salt, and $39 \%$ of $0.65 \%$ a $50 \mathrm{~g}$ sample would contain approximately $127 \mathrm{mg}$ of sodium. Therefore, the tested low sodium cheese sample did meet the "low sodium" CFR requirements.

Another major objective of this study was to determine if the emotional responses experienced when consuming a full sodium cheese can also be experienced when consuming a low sodium cheese. Based upon the results in section 5.4.4, the $100 \%$ and $50 \%$ Salona sample had the same caring response as the full sodium variable, $50 \% \mathrm{KCl}$ had a similar excited experience as the full sodium sample, and $100 \% \mathrm{KCl}$ and $50 \%$ Salona had the same judgmental experience as the full sodium, and $50 \% \mathrm{KCl}, 50 \%$ Salona, $100 \% \mathrm{KCl}$, and $100 \%$ Salona had similar sad experiences as the full sodium cheese, for the first day of testing; similar results were seen in the second day of testing. Overall, it appears similar emotions which are experienced in a full sodium cheese can also be experienced in a low sodium cheese; perhaps additional or more relevant emotions to the product category would provide further insight into how consumers emotionally relate to these nutritional alternative products compared to the traditional product.

Overall, the results of this study indicated the use of $100 \% \mathrm{KCl}$ (dipped in $0.257 \%$ SEA-i F75) may be the most appropriate and well liked sample after a full sodium mozzarella cheese. In regards to texture, higher mean scores of hardness, cohesiveness, gumminess, and chewiness were associated with cheeses which were most liked. Lastly, 
the cheeses with higher hedonic scores had increased changes in positive emotions and decreased changes in negative emotions, and the reverse was seen with disliked cheese products.

\subsection{Conclusion}

Decreasing the sodium content in mozzarella cheese has been a difficult undertaking for cheese manufacturers due to the traditionally mild flavor of the cheese. Partial substitution of $\mathrm{NaCl}$ with $\mathrm{KCl}$ has been relatively successful in other cheese varieties, and the current study determined the use of an approximately $1: 1$ ratio of $\mathrm{NaCl}$ to $\mathrm{KCl}$ provided a mozzarella cheese with similar saltiness and overall flavor liking to the full sodium control. Additionally, the emotional responses to the different cheeses were captured, and $100 \%$ Salona, $100 \% \mathrm{KCl}$, and $50 \% \mathrm{KCl}$ had relatively consistent similar emotional responses compared to the full sodium variable. Further research should investigate the use of additional and/or more relevant emotions to the food product category to determine whether a low sodium cheese is able to elicit similar emotional responses to those experienced when consuming the full sodium product. TPA indicated samples with higher means score in hardness, cohesiveness, gumminess, and chewiness were associated with the more liked cheese samples, thus providing additional information for manufacturers for developing a consumer acceptable low sodium mozzarella cheese. 


\section{$\underline{6.0}$}

\section{Conclusions}

Mozzarella cheese is the most highly consumed cheese in the United States, and appears to be a promising product for providing a low sodium option into the American diet. Decreasing one's sodium intake requires a modification of the entire diet; however, considering the per capita consumption rate of mozzarella cheese, an acceptable low sodium mozzarella cheese would likely make a beneficial dietary impact for those pursuing a lower sodium diet. Given the importance of a consumer acceptable low sodium product, researchers must go beyond traditional consumer testing methods in order to better understand how consumers feel about a product and any potential psychophysical effects a lower sodium mozzarella cheese might have on the consumer.

Through the research of this thesis, a sensory transparent antimicrobial was selected for use in a lower sodium cheese system, and the hedonic relationship between three salt replacers was determined. Based upon preliminary consumer testing, the results suggested the more acceptable salt replacer in a low sodium cheese was dependent upon what type of mozzarella cheese (direct acidification or bacteria cultured) was used.

The development, testing, and validation of a novel emotion and texture method (IMET) across three different product categories indicated the benefits of using consumer generated emotion images. The words only method had greater emotional changes in caring, self-confident, and sociable when compared to the My Pictures method. Additionally, the variability was greater for the words only for a positive emotion, while variability was greater for My Pictures in a negative emotion. Considering consumers 
were more likely to use images of themselves for positive emotions and images of others for negative emotions, it is hypothesized that consumers were better able to associate with the images of themselves (specifically in the positive emotions in the My Pictures method) compared to words only. Moving forward, perhaps consumers should be asked to use only images of themselves for helping to evoke the testing emotions and thus provide the most consistent results.

The consumer provided emotion image aspect of the IMET method was used in emotion and traditional consumer testing with non-commercial bacteria cultured low sodium mozzarella cheeses. The results of this study suggested the full sodium mozzarella cheese was consistently the most preferred samples, followed by the $100 \%$ $\mathrm{KCl}$ cheese, suggesting the use of $100 \% \mathrm{KCl}$ with SEA-i $\mathrm{F} 75$ at $0.275 \%$ would be the most appropriate formulation for a low sodium mozzarella cheese. The results also indicated cheese samples which where the most liked had greater increases in positive emotions over time while the least liked samples had greater increases in negative emotions over the consumption experience; additionally, similar emotions which were elicited in the full sodium cheeses were experience in some of the lower sodium cheeses. Texture analysis suggested cheese samples with the highest mean scores for all significant texture attributes were associated with the highest hedonic scored samples. Additionally, the low sodium cheese sample did meet the sodium level for making a "low sodium" label claim. Overall the series of tests in this thesis provide a better understanding of the role of antimicrobials and salt replacers in low sodium mozzarella cheese and consumer acceptability and emotional responses to this nutritional alternative product. 


\section{Future Research}

- Explore salt reward mechanisms at typically consumed levels with healthy to high levels of $\mathrm{NaCl}$ in the body.

- Descriptive sensory testing and broader consumer testing in low sodium mozzarella cheese is needed to understand key flavor characteristics, drivers of liking, and gain power on differences between samples.

- Investigate changes in emotion between males and females among similar sample population sizes and with a broader range of emotions.

- Investigate the relationship between a stimulus and emotion type (positive, neutral, and negative) and the potential for emotion specific products.

- Include "bitterness" and "aftertaste" in the hedonic questions whenever performing low sodium product testing. 


\section{$\underline{8.0}$}

\section{References}

Anker, M., Stading, M., \& Hermansson, A. M. (1999). Effects of pH and the gel state on the mechanical properties, moisture contents, and glad transition temperatures of whey protein films. Journal of Agricultural and Food Chemistry. 47, 1878-1886.

Anonymous. (1975). Minutes of Division Business Meeting. Institute of Food Technologists - Sensory Evaluation Division. In Stone. H., Bleibaum, R., \& Thomas, H. A. (2012). Sensory Evaluation Practices ( $4^{\text {th }}$ ed.). London, UK: Academic Press.

Ares, G., Gimenez, A., \& Gambaro, A. (2006). Preference mapping of texture of dulce de leche. Journal of Sensory Studies, 21(6), 553-571.

Ayyash, M. M., \& Shah, N. P. (2011). The effect of substitution of $\mathrm{NaCl}$ with $\mathrm{KCl}$ on chemical composition and functional properties of low-moisture Mozzarella cheese. Journal of Dairy Science, 94(8), 3761-3768.

Blechert, J., Feige, B., Hajcak, G., \& Tuschen-Caffier, B. (2010). To eat or not to eat? Availability of food modulates the electrocortical response to food pictures in restrained eaters. Appetite, 54(2), 262-268.

Bourne, M. C. (1978). Texture profile analysis. Journal of Food Technology, 32(7), 6266.

Brandt, M. A., Skinner, E. Z., \& Coleman, J. A. (1962). Texture profile method. Journal of Food Science, 28(4), 404-409.

Breene, W. M. (1975). Application of texture profile analysis to instrumental food texture evaluation. Journal of Texture Studies, 6, 53-82. 
Brody, L. R. (1997). Gender and emotion: Beyond stereotypes. Journal of Social Issues, 53(2), 369-393.

Brown, S., \& Ridderinkhof, K. R. (2009). Aging and the neuroeconomics of decision making: A review. Cognitive Affective \& Behavioral Neuroscience, 9(4), 365379.

CDC. (2012). Where's the sodium? There's too much in many common foods. Retrieved from http://www.cdc.gov/Features/VitalSigns/Sodium/.

Civille, G. V., \& Liska, I. H. (1974). Modifications and applications to foods of the General Foods sensory texture profile technique. Journal of Texture Studies, 6, $19-31$.

Collinsworth, L. A., Lammert, A. M., Ashman, H., Leidheiser, M., Garza, J., \& Keener, M. (2013). Development of a novel method for evaluating emotions and texture: Imagery versus language. Manuscript in preparation.

Costanzo, P. R., Musante, G. J., Friedman, K. E., Kern, L. S., \& Tomlinson, K. (1999). The gender specificity of emotional, situational, and behavioral indicators of binge eating in a diet-seeking obese population. International Journal of Eating Disorders, 26(2), 205-210.

Cromwell, H. C., \& Schultz, W. (2003). Effects of expectations for different reward magnitudes on neuronal activity in primate striatum. Journal of Neurophysiology, $89,2823-2838$.

Croy, I., Olgun, S., \& Joraschky, P. (2011). Basic emotions elicited by odors and pictures. Emotion, 11(6), 1331-1335. 
Cruz, A. G., Faria, J. A. F., Pollonio, M. A. R., Bolini, H. M. A., Celeghini, R. M. S., Granato, D., Shah, N. P. (2011). Cheeses with reduced sodium content: Effects on functionality, public health benefits and sensory properties. Trends in Food Science \& Technology, 22(6), 276-291.

Demott, B. J. (1985). Nutrient ratios in dairy products. Cultured Dairy Products Journal, 20(4), 6-9.

Desmet, P. M. A., Hekkert, P., \& Jacobs, J. J. (2000). When a car makes you smile: Development and application of an instrument to measure product emotions. Advances in Consumer Research, 27, 111-117.

Desmet, P. M. A., \& Schifferstein, H. N. J. (2008). Sources of positive and negative emotions in food experience. Appetite, 50(2-3), 290-301.

Drake, M. A., Miracle, R. E., \& McMahon, D. J. (2010). Impact of fat reduction on flavor and flavor chemistry of cheddar cheeses. Journal of Dairy Science, 93(11), 50695081.

Drake, S. L., Lopetcharat, K., \& Drake M. A. (2011). Salty taste in dairy foods: Can we reduce the salt? Journal of Dairy Science, 94:636-645.

Ekman, P., \& Friesen, W. V. (1986). A new pan-cultural facial expression of emotion. Motivation and Emotion, 10(2), 159-168.

El-Bakry, M., Beninati, F., Duggan, E., O'Riordan, E. D., \& O'Sullivan, M. (2011). Reducing salt in imitation cheese: Effects on manufacture and functional properties. Food Research International, 44(2), 589-596. 
Fern, E. 2009. Nestle's Salt Reduction Policy. Corporate Nutrition, Nestlé, Vevey, Switzerland.

Fitzgerald, E., \& Buckley, J. (1985). Effect of total and partial substitution of sodiumchloride on the quality of cheddar cheese. Journal of Dairy Science, 68(12), 31273134.

Foegeding, E. A., Daubert, C. R., Drake, M. A., Essick, G., Trulsson, M., Vinyard, C. J., \& Van de Velde, F. (2011). A comprehensive approach to understanding textural properties of semi- and soft-solid foods. Journal of Texture Studies, 42(2), 103129.

Freberg, L. (2009). Discovering biological psychology (2 ${ }^{\text {nd }}$ ed.). Belmont, CA: Wadsworth.

Food Labeling, 21 C.F.R. pt. 1 (2012).

Gard, M. G., \& Kring, A. M. (2007). Sex differences in the time course of emotion. Emotion, 7(2), 429-437.

Glimcher, P. W. (2001). Making choices: The neurophysiology of visual-saccadic decision making. Trends in Neurosciences, 24(11), 654-659.

Grummer, J., Karalus, M., Zhang, K., Vickers, Z., \& Schoenfuss, T. C. (2012). Manufacture of reduced-sodium cheddar-style cheese with mineral salt replacers. Journal of Dairy Science, 95(6), 2830-2839.

Guinee, T. P. (2004). Salting and the role of salt in cheese. International Journal of Dairy Technology, 57(2-3), 99-109.

Guinee. T. P., \& O'Kennedy, B. T. (2007). Reducing salt in foods: Practical strategies, (pp. 246-287). Boca Raton LA, USA: CRC Press. 
Holmes, E. A., Mathews, A., Mackintosh, B., \& Dalgleish, T. (2008). The causal effect of mental imagery on emotion assessed using picture-word cues. Emotion, 8(3), 395-409.

IDFA. (2012). Cheese Sales \& Trends. Retrieved October 2012, from http://www.idfa.org/news--views/media-kits/cheese/cheese-sales-and-trends/.

Jaeger, S. R., Jørgensen, A. S., Aaslyng, M. D., \& Bredie, W. L. (2008). Best-worst scaling: An introduction and initial comparison with monadic rating for preference elicitation with food products. Food Quality and Preference, 19(6), $579-588$.

Jaeger, S. R. (2012). Fifth EuroSense Conference; Bern, Switzerland.

JMP. (2012). Nonparametric: Descriptions of nonparametric tests. Retrieved December 2012, from http://www.jmp.com/support/help/Nonparametric.shtml\#658209.

Johnson, M. E., Kapoor, R., McMahon, D. J., McCoy, D. R., \& Narasimmon, R. G. (2009). Reduction of sodium and fat levels in natural and processed cheeses: Scientific and technological aspects. Comprehensive Reviews in Food Science and Food Safety, 8(3), 252-268.

Katsiari, M. C., Voutsinas, L. P., Alichanidis, E., \& Roussis, I. G. (1997). Reduction of sodium content in feta cheese by partial substitution of $\mathrm{NaCl}$ by $\mathrm{KCl}$. International Dairy Journal, 7(6-7), 465-472.

Kenny, P. J. (2011). Reward Mechanisms in Obesity: New Insights and Future Directions. Neuron, 69(4), 664-679. 
King, S. C., \& Meiselman, H. L. (2010). Development of a method to measure consumer emotions associated with foods. Food Quality and Preference, 21(2), 168-177.

King, S. C., Meiselman, H. L., \& Carr, B. T. (2010). Measuring emotions associated with foods in consumer testing. Food Quality and Preference, 21(8), 1114-1116.

Kring, A. M., \& Gordon, A. H. (1998). Sex differences in emotion: Expression, experience, and physiology. Journal of Personality and Social Psychology, 74(3), 686-703.

Kringelbach, M. L. (2009). Neural basis of mental representations of motivation, emotion, and pleasure. Handbook of Neuroscience for the Behavior Sciences.

Lawless, H.T., \& Heymann, H. (1998). Sensory evaluation of food: Principals and practices. New York: Chapman and Hall.

Li, J. R., Yan, J. Q., Chen, K., Lu, B., Wang, Q., Yan, W., et al. (2012). Lesions of the central nucleus of the amygdala decrease taste threshold for sodium chloride in rats. Brain Research Bulletin, 89(1-2), 8-15.

Lucassen, M. P., Gevers, T., \& Gijsenij, A. (2011). Texture affects color emotion. Color Research and Application, 36(6), 426-436.

Macht, M. (1999). Characteristics of eating in anger, fear, sadness and joy. Appetite, 33(1), 129-139.

Macht, M. (2008). How emotions affect eating: A five-way model. Appetite, 50(1), 1-11.

Macht, M., Gerer, J., \& Ellgring, H. (2003). Emotions in overweight and normal-weight women immediately after eating foods differing in energy. Physiology \& Behavior, 80(2-3), 367-374.

Macht, M., \& Simons, G. (2000). Emotions and eating in everyday life. Appetite, 35(1), 
$65-71$.

Martin-Soelch, C., Linthicum, J., \& Ernst, M. (2007). Appetitive conditioning: Neural bases and implications for psychopathology. Neuroscience and Biobehavioral Reviews, 31(3), 426-440.

McCaughey, S. A., \& Scott, T. R. (1998). The taste of sodium. Neuroscience and Biobehavioral Reviews, 22(5), 663-676.

McNair, F. M., Lorr, M., \& Droppleman, L. F. (1971). Profile of mood states. San Diego: Educational and Industrial Testing Service.

Meilgaard, M. C., Civille, G. V., \& Carr, B. T. (1991). Sensory evaluation techniques $\left(2^{\text {nd }}\right.$ ed.). Baton Rouge, LA: CRC Press.

Meilgaard, M. C., Civille, G. V., \& Carr, B. T. (2007). Sensory evaluation techniques $\left(4^{\text {th }}\right.$ ed.). Baton Rouge, LA: CRC Press.

Moskowitz, H.R. (1987). Food Texture: Instrumental and sensory measurement. New York, NY: Marcel Dekker, Inc.

Mozzarella cheese and scamorza cheese, 21 C.F.R. pt. 1 (1993).

Murray, J. M., Delahunty, C. M., \& Baxter, I. A. (2001). Descriptive sensory analysis: Past, present and future. Food Research International, 34(6), 461-471.

National Dairy Council. (2011). Health Professional Cheese Nutrition. Retrieved October, 2012 from http://www.nationaldairycouncil.org/SiteCollectionDocuments/education_materia 1s/cheese/Health\%20Professional\%20Cheese\%20Nutrition\%20Brochure\%20Final .pdf. 
NHLBI. (2012). What is high blood pressure? Retrieved October, 2012 from http://www.nhlbi.nih.gov/health/health-topics/topics/hbp/.

O'Doherty, J. P., Deichmann, R., Critchley, H. D., \& Dolan, R. J. (2002). Neural responses during anticipation of a primary taste reward. Neuron, 33(5), 815-826.

Piech, R. M., Pastorino, M. T., \& Zald, D. H. (2010). All I saw was the cake. Hunger effects on attentional capture by visual food cues. Appetite, 54(3), 579-582.

Pons, M., \& Fiszman, S. M. (1996). Instrumental texture profile analysis with particular reference to gelled systems. Journal of Texture Studies, 27(6), 597-624.

Reddy, K. A., \& Marth, E. H. (1993). Composition of cheddar cheese made with sodium chloride and potassium chloride either singly or as mixtures. Journal of Food Composition and Analysis, 6, 354-363.

Roessler, E. B., Pangborn, R. M., Sidel, J. L., \& Stone, H. (1978). Expanded statistical tables for estimating significance in paired-preference, paired-difference, duo-trio, and triangle tests. Journal of Food Science, 43(3), 940-943.

Rolls, B. J., Rolls, E. T., Rowe, E. A., \& Sweeney, K. (1981). Sensory specific satiety in man. Physiology \& Behavior, 27(1), 137-142.

Rolls, E. T. (1997). Taste and olfactory processing in the brain and its relation to the control of eating. Critical Reviews in Neurobiology, 11(4), 263-287.

Rolls, E. T. (1999). The functions of the orbitofrontal cortex. Neurocase, 5(4), 301-312.

Rolls, E. T., Browning, A. S., Inoue, K., \& Hernadi, I. (2005). Novel visual stimuli activate a population of neurons in the primate orbitofrontal cortex. Neurobiology of Learning and Memory, 84(2), 111-123.

Rolls, E. T., \& Grabenhorst, F. (2008). The orbitofrontal cortex and beyond: From affect 
to decision-making. Progress in Neurobiology, 86(3), 216-244.

Rolls, E. T., Hornak, J., Wade, D., McGarth, J. (1994). Emotion-related learning in patients with social and emotional changes associated with frontal lobe damage. Journal of Neurology, Neurosurgery, and Psychiatry, 57, 1518-1524.

Saltos, E. S., \& Bowman, S. (1997). Dietary guidance on sodium: Should we take it with a grain of salt? Nutrition Insights, USDA.

Sander, D. (2012). The role of the amygdala in the appraising brain. Behavioral and Brain Sciences, 35(3), 161.

Schifferstein, H. N. J., \& Desmet, P. M. A. (2010). Hedonic asymmetry in emotional responses to consumer products. Food Quality and Preference, 21(8), 1100-1104.

Schroeder, C. L., Bodyfelt, F. W., Wyatt, C. J., \& McDaniel, M. R. (1988). Reduction of sodium chloride in cheddar cheese: Effect on sensory, microbiological, and chemical properties. Journal of Dairy Science, 71(8), 2010-2020.

Scott, T. R., Karadi, Z., Oomura, Y., Nishino, H., Platasalaman, C. R., Lenard, L., Giza, B. K., Aou, S. (1993). Gustatory neural coding in the amygdala of the alert macaque monkey. Journal of Neurophysiology, 69(6), 1810-1820.

Seo, H. S., Lee, Y., Yoon, N. R., Song, J. M., Shin, J. M., Lee, S. Y., Hwang, I. (2009). Impacts of sensory attributes and emotional responses on the hedonic ratings of odors in dairy products. Appetite, 53(1), 50-55.

Spinelli, S. (2012). Fifth EuroSense Conference; Bern, Switzerland.

Stone. H., Bleibaum, R., \& Thomas, H. A. (2012). Sensory Evaluation Practices (4 ${ }^{\text {th }}$ ed.). London, UK: Academic Press. 
Thomson, D. M. H., \& Crocker, C. (2011). A data-driven classification of states of mind and an appraisal of the role played by state of mind in choice behavior [O9.1 Abstract]. Pangborn Symposium.

Thomson, D. M. H., Crocker, C., \& Marketo, C. G. (2010). Linking sensory characteristics to emotions: An example using dark chocolate. Food Quality and Preference, 21(8), 1117-1125.

USDA. (2010). Dietary Guidelines for Americans: Chapter 8: Sodium and potassium. US Department of Health and Human Services; USDA. Retrieved October, 2012 from http://www.healthierus.gov/dietaryguidelines.

USDA. (2010). Economic Research Service: Background. Retrieved October, 2012 from http://www.ers.usda.gov/topics/animal-products/dairy/background.aspx.

Zaltman, G., \& Coulter, R. H. (1995). Seeing the voice of the customer: Metaphor-based advertising research. Journal of Advertising Research, 35(4), 35-51.

Zuckerman, M., \& Lubin, B. (1965). Normative data for the multiple affect adjective check list. Psychological reports, 16(2), 438-438. 